\title{
The Structure of Jets at Hadron Colliders
}

\author{
By Andrew James Larkoski
}

Thesis 
THE STRUCTURE OF JETS AT HADRON COLLIDERS

\author{
A DISSERTATION \\ SUBMITTED TO THE DEPARTMENT OF PHYSICS \\ AND THE COMMITTEE ON GRADUATE STUDIES \\ OF STANFORD UNIVERSITY \\ IN PARTIAL FULFILLMENT OF THE REQUIREMENTS \\ FOR THE DEGREE OF \\ DOCTOR OF PHILOSOPHY
}

Andrew J. Larkoski

August 2012 


\section{Acknowledgements}

Foremost, I must thank my very patient advisor, Michael Peskin, for his guidance, assistance and criticisms. I have learned an enormous amount about physics and how to be a physicist from him.

My fellow graduate students have been an invaluable resource for learning as well as maintaining sanity in graduate school. I thank my collaborators, Martin Jankowiak and Camille Boucher-Veronneau, for the opportunity to work with them. I also thank Jeffrey Pennington, Tomas Rube, Eder Izaguirre, Randy Cotta, Kassahun Betre, Daniel Harlow and Spencer Gessner for valuable conversations about physics and, more importantly, about things other than physics.

I have also been blessed to communicate with numerous post-docs, both at SLAC and from other institutions. I thank Jared Kaplan, Jonathan Walsh, Michael Spannowsky and Chris Vermilion for guidance and advice.

Other SLAC faculty have assisted me by writing letters of recommendation, reviewing drafts of papers, or serving on committees so that I can graduate. I thank JoAnne Hewett, Lance Dixon, Jay Wacker, Shamit Kachru, Ariel Schwartzman and Stan Brodsky for their patience and constructive criticisms.

In addition, I have had the great honor of attending many conferences and giving many seminars where I have met numerous physicists from which I have learned an enormous amount. Steve Ellis, Matt Strassler, Peter Skands, Peter Loch, Michael Seymour and David Kosower have been instrumental in helping me think outside the Stanford bubble.

During the summer of 2010, I had the great fortune of attending the Cargèse Summer School in Corsica, France, where I met and befriended many other physics 
graduate students from around the world (as well as enjoying the beaches and mountains of Corsica). Because of this school, I started an international collaboration with students and faculty I met at the school. I thank the organizers Geraldine Servant, Christophe Grojean and Jean Orloff for an incredible school.

During graduate school, I have been funded generously by the Department of Energy and Stanford University Physics Department as a student at SLAC National Accelerator Lab. I also received a travel award from the LHC Theory Initiative, which allowed me to travel internationally several times to attend conferences and meet with collaborators. I graciously thank all of these organizations for their support.

I cannot thank enough my wife and best friend, Patty, for her tolerance, encouragement, silliness and cooking(!) when graduate school became a bit overbearing.

Finally, I thank my Mom, Dad and Scott for their unwavering support for my education throughout my life. While you may not have always understood what I was doing at school, you've always demanded the best from me which has had an indelible effect on my studies. 


\section{Contents}

\begin{tabular}{|ll}
\hline Acknowledgements & v
\end{tabular}

1 Introduction 1

1.1 Overview . . . . . . . . . . . . . . . . . . . . . . . 1

1.2 Modeling Proton Collisions . . . . . . . . . . . . . . . . . . . . 3

1.3 Jets $\ldots \ldots \ldots \ldots \ldots \ldots \ldots$

1.4 Outline. . . . . . . . . . . . . . . . . . . . . 9

I New Models for Jet Structure 11

\begin{tabular}{|lll}
2 & Spin-Dependent Antenna Splitting Functions & 12
\end{tabular}

2.1 Introduction . . . . . . . . . . . . . . . . . . . . . . . . . . . . . . . . 12

2.2 Proposal for the $2 \rightarrow 3$ splitting functions . . . . . . . . . . . . . 14

2.3 Spin-0 case . . . . . . . . . . . . . . . . . . . . . . . . . . 19

2.4 Spin-1 and spin-2 case . . . . . . . . . . . . . . . . . 23

2.5 Spin- $\frac{1}{2}$ and spin- $\frac{3}{2}$ cases . . . . . . . . . . . . . . . . . 26

2.6 Initial-state showers . . . . . . . . . . . . . . . . . . . . . . . 29

2.7 Comparison to previous results . . . . . . . . . . . . . . . . . . . 37

3 Antenna Splitting Functions for Massive Particles 46

3.1 Introduction . . . . . . . . . . . . . . . . . . 46

3.2 The spin zero fermion-quark antenna . . . . . . . . . . . . . . . . . . 49

3.3 Kinematics of massive antennae . . . . . . . . . . . . . . . . . . . . . 57 


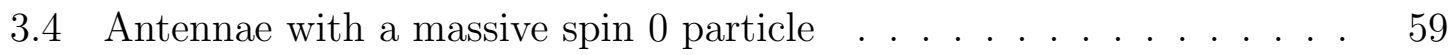

3.5 Antennae with a massive spin $\frac{1}{2}$ particle . . . . . . . . . . . . 61

3.6 Antennae of a pair of massive particles . . . . . . . . . . . . 66

3.7 Antennae with massive particle production . . . . . . . . . . . . 70

3.8 Conclusion $\ldots \ldots \ldots \ldots$. . . . . . . . . . . . . . . . . . . . . 72

II New Observables for Jet Structure 73

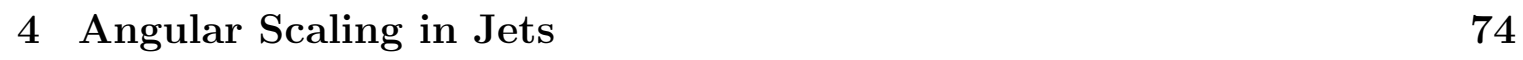

4.1 Introduction . . . . . . . . . . . . . . . . . . 74

4.2 Average angular structure function . . . . . . . . . . . . . . . . 75

4.3 Calculating the average . . . . . . . . . . . . . . . . . . . . . 78

4.3 .1 Collinear approximation . . . . . . . . . . . . . 79

4.3 .2 Running coupling . . . . . . . . . . . . . . . 80

4.3 .3 Higher order effects . . . . . . . . . . . . . . . . . . . . 81

4.3 .4 Factorization . . . . . . . . . . . . . . . . 85

4.4 Effect of uncorrelated radiation . . . . . . . . . . . . . 85

4.4 .1 Background . . . . . . . . . . . . . . . 86

4.4 .2 Procedure . . . . . . . . . . . . . . . . . . . . . . . . . . 88

4.4 .3 Results $\ldots \ldots \ldots \ldots$

4.5 Angular correlations in the transverse region . . . . . . . . . . . . . . 91

4.5 .1 Feynman-Wilson Gas . . . . . . . . . . . . . . . . . 92

4.5 .2 Toy Monte Carlo . . . . . . . . . . . . . . . . . . . . . . 93

4.5 .3 Emergence of jets . . . . . . . . . . . . . . . 95

4.6 Discussion and conclusions $\ldots \ldots \ldots$. . . . . . . . . . . . . . . . . . . . . . . 95

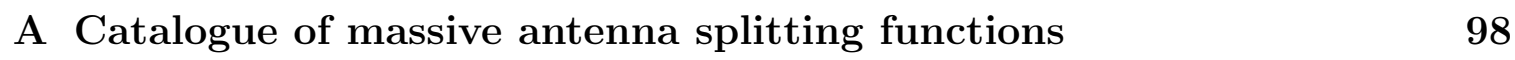

A.0.1 Splitting functions with one massive scalar . . . . . . . . . . . 98

A.0.2 Splitting functions with one massive fermion . . . . . . . . . . 99

A.0.3 Splitting functions with two massive scalars . . . . . . . . . . 102

A.0.4 Splitting functions with a massive fermion and a massive scalar 102 
A.0.5 Splitting functions with two massive fermions . . . . . . . . . 102

A.0.6 Splitting functions with pair production of scalars . . . . . . . 104

A.0.7 Splitting functions with pair production of fermions . . . . . . 104

\begin{tabular}{llll}
\hline B Spin-dependent Altarelli-Parisi functions for massive particles & 107
\end{tabular}

\begin{tabular}{ll}
\hline C Monte Carlo & 109 \\
\hline
\end{tabular} 


\section{List of Tables}

$2.1 \quad$ Numerator functions $\mathcal{N}\left(z_{a}, z_{b}, z_{c}\right)$ for the spin-dependent $2 \rightarrow 3$ splitting functions $A B \rightarrow a c b: \mathcal{S}=\mathcal{N} /\left(y_{a b} y_{a c} y_{b c}\right)$. Each line gives a choice of $A B$. The labels denote the polarization of the three final particles with the radiated particle $c$ in the center: $\left(h_{a}, h_{c}, h_{b}\right)$. The empty columns are forbidden by quark chiral symmetry. By the $\mathrm{P}$ and $\mathrm{C}$ invariance of QCD, the same expressions apply after exchanging $-\leftrightarrow+$, $q \leftrightarrow \bar{q}$, or $A B a c b \leftrightarrow B A b c a$. . . . . . . . . . . . . . . . . . . . . . . 18

$2.2 \quad$ Spin-dependent Altarelli-Parisi splitting functions $P(z)$ for splittings $B \rightarrow c b$. The labels denote the polarization of the two final particles with the radiated particle first: $\left(h_{c}, h_{b}\right)$. The empty columns are forbidden by quark chiral symmetry. By the $\mathrm{P}$ and $\mathrm{C}$ invariance of QCD, the same expressions apply after exchanging $-\leftrightarrow+$ or $q \leftrightarrow \bar{q}$. . . . . 19

4.1 Table of extracted UE densities. $\Lambda_{\text {Trans }}$ is the average $p_{T}$ density in the transverse region and $\bar{\Lambda}_{\mathrm{UE}}$ is extracted from $\langle\Delta \mathcal{G}(R)\rangle$. The 'error' bars quoted for $\bar{\Lambda}_{\mathrm{UE}}$ are the maximum difference of the function $\Lambda_{\mathrm{UE}}(R)$ from its average, which is computed in the range from $R=0.4$ to $R=0.8$. Tunes $4 \mathrm{C}^{\prime}$ and $4 \mathrm{C}^{\prime \prime}$ differ from tune $4 \mathrm{C}$ in that they have

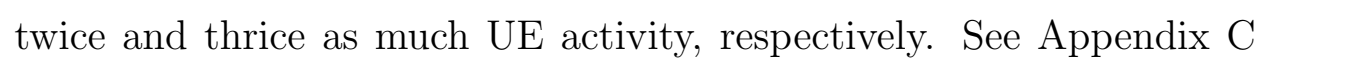
for more details about the Monte Carlo. . . . . . . . . . . . . . . . . 90 


\section{List of Figures}

2.1 (a) Kinematics of $2 \rightarrow 3$ splitting in the final state (FF) case. (b) Phase space for $2 \rightarrow 3$ splitting in the FF case. The six regions corresponding to different orderings of $s_{a b}, s_{a c}, s_{b c}$ are shown. The region that should be well described by an antenna splitting $A B \rightarrow a c b$ is shaded. . . . . 15

$2.2 \quad$ Feynman diagrams for the computation of the $g g \rightarrow g g g$ splitting

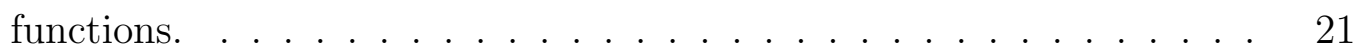

$2.3 \quad$ Feynman diagram for the computation of the $g g \rightarrow \bar{q} q g$ splitting functions. . . . . . . . . . . . . . . . . . . 22

$2.4 \quad$ Feynman diagrams for the computation of the $q g \rightarrow q g g$ splitting functions. . . . . . . . . . . . . . . . . . 26

2.5 (a) Kinematics of $2 \rightarrow 3$ splitting in the initial-final (IF) case. (b) Phase space for $2 \rightarrow 3$ splitting in the IF case. The eight regions corresponding to different orderings of $\left|s_{a b}\right|,\left|s_{a c}\right|, s_{b c},\left|Q^{2}\right|$ are shown. The region that should be well described by an antenna splitting $A B \rightarrow$ $a c b$ is shaded. . . . . . . . . . . . . . . . . . . 31

2.6 (a) Kinematics of $2 \rightarrow 3$ splitting in the initial state (II) case. (b) Phase space for $2 \rightarrow 3$ splitting in the II case. The six regions corresponding to different orderings of $\left|s_{a c}\right|,\left|s_{b c}\right|,\left|Q^{2}\right|$ are shown. The region that should be well described by an antenna splitting $A B \rightarrow a c b$ is shaded. 34 
2.7 Visualization of the ratio of the ARIADNE antenna function to our antenna functions for the processes $g g \rightarrow g g g$. The figures on the left and right are the comparison of the ARIADNE antenna function to our spin-summed antenna functions from row 1 and row 2 in Table $\mid 2.1$. respectively. The boundaries of phase space for the different kinematic regions are marked in blue. The contours are plotted at ratios of 1.2, $1.5,2.0,3.0$, and 5.0, with + indicating a region in which the ratio is greater than $1 . \ldots \ldots \ldots \ldots$. . . . . . . . . . . . 40

2.8 Visualization of the ratio of the ARIADNE antenna function to our antenna function for the process $q_{-} \bar{q}_{-} \rightarrow q g \bar{q}$. Our antenna function for the process $q_{-} \bar{q}_{+} \rightarrow q g \bar{q}$ coincides with the ARIADNE result and so is not included. The notation is as in Fig. $\mid 2.7$. . . . . . . . . . 41

2.9 Visualization of the ratio of the ARIADNE antenna function to our antenna functions for the processes $q g \rightarrow q g g$. The figures on the left and right are the comparison of the ARIADNE antenna function to our spin-summed antenna functions from row 7 and row 8 in Table 12.1, respectively. The notation is as in Fig. [2.7] . . . . . . . . 41

2.10 Visualization of the ratio of the Gehrmann-De Ridder antenna function to our antenna function for the process $g_{-} g_{+} \rightarrow g g g$. The antenna function for the process $g_{+} g_{+} \rightarrow g g g$ coincides with the Gehrmann-De Ridder result and so is not included. The notation is as in Fig. [2.7. . 43

2.11 Visualization of the ratio of the Gehrmann-De Ridder antenna functions to our antenna functions for the processes $q g \rightarrow q g g$. The figures on the left and right are the comparison of the Gehrmann-De Ridder antenna function to our spin-summed antenna functions from row 7 and row 8 in Table 2 2.1, respectively. The notation is as in Fig. 2.7. . . 44

2.12 Visualization of the ratio of the Gehrmann-De Ridder antenna functions to our antenna functions for the processes $q g \rightarrow q q \bar{q}$. The figures on the left and right are the comparison of the Gehrmann-De Ridder antenna function to our spin-summed antenna functions from row 9 and row 10 in Table 2.1 , respectively. The notation is as in Fig. [2.7]. $\quad 45$ 
3.1 Feynman diagrams for the computation of the $F g \rightarrow F g g$ splitting functions [39]. . . . . . . . . . . . . . . . . . . . . . . . . . . . . . . . 64

3.2 The single Feynman diagram for the computation of the $g g \rightarrow g F \bar{F}$ splitting function [39]. . . . . . . . . . . . . . . 70

$4.1 \quad$ Splitting diagram for the $\mathcal{O}\left(\alpha_{s}^{2}\right)$ contribution to $\langle\mathcal{G}(R)\rangle$. . . . . . . . 82

4.2 Average angular structure functions for ensembles of jets with $p_{T}>$ $200 \mathrm{GeV}$ and no underlying event or initial state radiation. Red curves denote quark jets and blue curves denote gluon jets. These are antikT jets with jet radius $R_{0}=1.0$ as generated with Pythia8 (left) and Herwig++ (right). See Appendix $\mid \mathrm{C}$ for more details about the Monte Carlo. . . . . . . . . . . . . . . . . . . 83

4.3 Average angular structure functions for three different ensembles of jets with $p_{T}>200 \mathrm{GeV}$ and no underlying event or initial state radiation. These are anti-kT jets with jet radius $R_{0}=1.0$ as generated with Pythia8 (solid) and Herwig++ (dashed). See Appendix $\mathrm{C} \mid$ for more details about the Monte Carlo. . . . . . . . . . . . . . . . . . . . . . . 84

4.4 Average angular structure functions for ensembles of jets with $p_{T}>$ $200 \mathrm{GeV}$. The black curves have no underlying event nor any ISR. In contrast to the previous section, here underlying event and ISR are turned on for the colored curves. On the LHS, the Pythia8 samples make use of tune $4 \mathrm{C}$, with the red curve having twice as much UE activity as the blue curve. On the RHS, the purple curve corresponds to Herwig++ tune LHC-UE7-2. For comparison, the bottom figure overlays the Pythia8 and Herwig ++ curves. These are anti-kT jets with jet radius $R_{0}=1.0$. See Appendix $\mid \mathrm{C}$ for more details about the Monte Carlo . . . . . . . . . . . . . . . . . . . . . . . . . 87 
\begin{tabular}{|ll}
4.5 & $\Lambda_{\mathrm{UE}}(R)$ curves as extracted via the procedure detailed in Sec. \\
4.4.2.
\end{tabular} The solid curves correspond to the Pythia8 samples in Fig. 4.4, while the dashed curve corresponds to the Herwig++ sample. The dotted curve corresponds to the same Herwig++ sample as the dashed curve, the difference being that the power law of the underlying event ansatz is changed from $R^{4}$ to $R^{3.3}$. The matching was done at $R_{\min }=0.25$. $\quad 88$

4.6 Definition of the transverse region. . . . . . . . . . . . . . . . . . 91

$4.7\langle\Delta \mathcal{G}(R)\rangle$ in the trans-min region. The black curve corresponds to the Feynman-Wilson gas. The purple curve corresponds to Pythia8 dijet events with $p_{\text {Tjet }}>200 \mathrm{GeV}$. The red curve corresponds to the toy Monte Carlo, described below. Finally, the blue curve corresponds to an ensemble of single "DLA mini-jets" as employed in the toy Monte Carlo. See Appendix $\mid$ C for more details about the Monte Carlo. . . . 93

$4.8\langle\Delta \mathcal{G}(R)\rangle$ for ensembles excluding events where the $p_{T}$ of the hardest charged particle in the trans-min region exceeds a given $p_{T \max }$. $\langle\Delta \mathcal{G}(R)\rangle$ for the Feynman-Wilson gas is shown in black, while the colored curves correspond to Pythia8 dijet events with $p_{\text {Tjet }}>200 \mathrm{GeV}$. The purple curve has no $p_{T}$ cut, while the blue, green, orange and red curves correspond to $p_{T \max }$ of $16 \mathrm{GeV}, 8 \mathrm{GeV}, 4 \mathrm{GeV}$ and $1 \mathrm{GeV}$, \begin{tabular}{l|l|l|l|} 
respectively. See Appendix & for more details about the Monte Carlo. & 94
\end{tabular} 


\section{Chapter 1}

\section{Introduction}

\subsection{Overview}

Particle physics seeks to understand the interactions and properties of the fundamental particles. To gain understanding, there is an interplay between theory and experiment. Models are proposed to explain how particles behave and interact. These models make precise predictions that can be tested. Experiments are built and executed to measure the properties of these particles, providing necessary tests for the theories that attempt to explain the realm of fundamental particles. However, there is also another level of interaction between theory and experiment; the development of new experiments demands the study of how particles will behave with respect to the measured observables toward the goal of understanding the details and idiosyncrasies of the measurements very well. Only once these are well-modeled and understood can one be confident that the data that are measured is trustworthy.

To study these smallest of particles, enormous experiments are built to probe at smaller and smaller distance scales. The program of particle physics experiments, since at least E. O. Lawrence and the cyclotron in the 1930s, has been to collide highly energetic particles and observe the debris. While this procedure may seem somewhat Neanderthal, it is very effective: particles will have a signature visible in the "debris" that is observed. Each particle physics experiment will create different particles with signatures that depend in detail on, for example, which particles are 
being collided or how the debris is being measured. Thus, for each experiment, it is necessary and vital to determine the signatures of particular particles that may be observed so as to search for these signals.

The most recent and powerful of these experiments is the Large Hadron Collider (LHC), in Geneva, Switzerland, which accelerates protons to enormous energies, collides them and records the particles that are created in the collision. Each colliding proton has approximately the kinetic energy of a flying mosquito, which itself contains about $10^{20}$ protons. At these energies it is believed that the experiments at the LHC can probe energies that have not existed in the universe since shortly after the Big Bang, and, as such, test our models of physics at these high energies. For example, the Higgs boson, the last undiscovered piece of the Standard Model of particle physics, should, if it exists, be observed at the LHC.

The two general-purpose experiments at the LHC, ATLAS [1, 2] and CMS[3], are enormous detectors designed to capture all of the energy that is released in a protonproton collision. To do this, there are many different pieces; the detectors have trackers to measure the path of individual charged particles created in the collision; finely-segmented calorimeters to measure with high precision the energy of particles in a particular direction; and, magnetic fields throughout the detectors to bend the charged particles so that their charge and momentum can be measured. From each proton-proton collision, there is a huge amount of information that is recorded that must be interpreted to probe what occured at the scale of the proton collision.

However, all of this comes at a price. Proton collisions are very messy environments from the standpoint of a particle detector. Protons are not fundamental particles; they are composed of more fundamental quarks and gluons which carry the strong or color force, quantum chromodynamics (QCD). Collectively, quarks and gluons are called "partons". It is actually the partons that collide and create the observed particles. However, because protons are not elementary, there can be multiple collisions of quarks and gluons in the protons, gluon radiation, and other processes that substantially muddy the data that are collected. Thus, it is necessary to have good models of proton collisions and the subsequent processes to be able to separate signals of the Higgs boson, for example, from the overwhelming and messy physics of 
proton collisions. The modeling and interpretation of the physics of a proton collider, such as the LHC, is the main topic of this thesis.

\subsection{Modeling Proton Collisions}

The model of proton collisions is based on the principle of factorization: the principle that physics at widely disparate energy or distance scales decouples from one another and can be studied individually. Using the principle of factorization, the proton collision can be broken up into several pieces which can be analyzed sequentially:

1. Protons are accelerated to very high energies and are made to collide.

2. At collision, individual quarks and gluons in the protons interact, exchanging a large amount of momentum and producing a number of energetic particles. This is called the "hard interaction".

3. The partons in the hard collision radiate additional particles before or after the hard interaction takes place. The radiation from partons before the hard collision is called "initial state radiation" (ISR). The energetic particles that are created in the hard interaction or from ISR may be charged under QCD and as such can radiate additional quarks or gluons. This radiation is called "final state radiation" (FSR). Working outward from the hard collision, the radiation from ISR and FSR can be modeled collectively. This is called the "parton shower".

4. Unstable particles created from the hard interaction can decay.

5. The colored particles that exist after the parton shower "hadronize", forming colorless bound states. Hadronization occurs because QCD is a confining interaction in which the individual colored partons do not exist freely but only as bound in colorless particles called hadrons. The parton shower is allowed to continue until the energy of the particles created in the shower is comparable to the scale of the formation of colorless bound states, around an energy of 1 giga-electron Volt $(\mathrm{GeV})$. 
6. Spectator partons in the colliding protons that did not participate in the hard collision interact, creating a nearly uniform distribution of soft, low energy particles. This is called the "underlying event".

7. Stable or long-lived hadrons are observed in the detector.

Factorization allows for this separation of the physics of the proton collision because to each of the points above there is a distinct energy and time scale associated with it. For example, the hard interaction is at a much higher energy than the confining scale of QCD, so there is a large dynamic range for the parton shower to produce particles. This thesis will focus on studying the physics of the parton shower and its consequences.

To understand the model of the parton shower, one needs to understand the structure of QCD. QCD is a non-Abelian gauge theory with gauge symmetry SU(3). Quarks are in the fundamental representation of SU(3) and gluons, as vector bosons and the force carrier of QCD, are in the adjoint representation. For invariance of QCD under SU(3) rotations in color space, there are three-point interactions between quarks and gluons and three- and four-point interactions between gluons. These interactions are responsible for the production of quark and gluon radiation. The probability of a quark or gluon radiating depends on the momentum of the radiated particle. Due to the non-Abelian structure of QCD, the probability for radiating soft (low energy) or collinear particles is enhanced with respect to other kinematic configurations. A sequence of soft and collinear emissions can occur starting from a high energy quark or gluon created in the hard interaction producing a collimated stream of particles. At very high energies, QCD is approximately scale-invariant, so the structure of this sequence of soft and collinear emissions should also be scaleinvariant. This is the model of the parton shower and the construction of "jets": collimated, energetic streams of particles that are approximately scale-invariant. Jets are ubiquitous at hadron colliders because of the large number of colored particles that exist.

The standard way to study QCD is through perturbation theory. The coupling in QCD of quarks to gluons, $\alpha_{s}$, is relatively small and so it is sensible to compute 
predictions for experiments as Taylor series in the QCD coupling. In addition, because QCD is a non-Abelian gauge theory, there is also an expansion in the number of colors, or, rather, in the rank of the gauge group. For QCD, because the gauge group is $\mathrm{SU}(3)$, the number of colors is 3. It has been known since G. 't Hooft in the 1970s [4] that the expansion in the rank of the gauge group can be interpreted as an expansion in the topology of the color flow in a process; in particular, as an expansion in the Euler characteristic of the two-dimensional diagram representing the color flow. The topology of the color flow simplifies substantially in the limit that the number of colors tends to infinity; thus, there is also and expansion in QCD in inverse powers of the rank of the gauge group. The term "leading color" refers to considering the contributions to QCD which are of highest order in the number of colors. Topologically, this means that color flow diagrams are planar.

Parton showers employ both of these approximations to simulate the radiation created from high energy quarks and gluons created in a hard interaction. The fact that QCD is perturbative allows the parton shower to be well-approximated by the simplest radiation processes; namely, a quark or gluon radiating a single particle. The color expansion of QCD allows the parton shower to good approximation to assume that the radiation is local in color space. That is, one can attribute radiation (almost) uniquely to a radiator from which it originated. These assumptions lead to enormous simplifications but, perhaps surprisingly, predict data with astonishing accuracy.

There is another assumption and approximation in the parton shower that is tied to the enhancement of soft and collinear radiation with respect to other radiation in QCD. Generically, in quantum field theories, processes that persist over a wide range of energy scales produce logarithms of ratios of scales. For example, in the case of the parton shower, the particles of the hard interaction have large energy $Q$, while the radiation from them will be at much lower energies; call these $E$. This fact leads to factors of $\log Q / E$ enhancements of the production of soft, low energy particles from radiation. To be able to describe the physics of the soft radiation well, these logarithms must be understood with care. Soft and collinear radiation can occur in a sequential manner with two emissions of soft radiation enhanced by a factor of $\log ^{2} Q / E$. An arbitrary number of soft particles can be radiated leading to the 
necessity to resum these logarithmic enhancement factors. Only once resummation is done can predictions be trusted for processes or regimes that are sensitive to soft and collinear radiation.

The way in which the resummation of large logarithms is accomplished in the parton shower is, effectively, by allowing for an arbitrary number of soft or collinear particles to be created in the radiation of a particle created in the hard interaction. This is accomplished by the Sudakov form factor, $\Delta\left(Q, Q_{0}\right)$, which is the probability that there is no emission of radiation from the scale $Q_{0}$ to $Q$. The Sudakov form factor can be expressed as

$$
\Delta\left(Q, Q_{0}\right)=\exp \left\{-\int_{Q}^{Q_{0}} \frac{d Q^{\prime}}{Q^{\prime}} \frac{\alpha_{s}}{2 \pi} P\left(Q^{\prime}\right)\right\}
$$

where the kernel of the Sudakov form factor, $P\left(Q^{\prime}\right)$, is called the splitting function. The splitting function encodes how the radiation is created in the parton shower. In QCD, it is a function that is universal in the sense that it is independent of the physics of the hard interaction in a proton collision and only depends on the radiating and radiated particles. Typically, the splitting functions encode the leading logarithms: the logarithmic terms that are the largest for soft or collinear emissions. While the splitting functions are universal, the definition of the scale $Q$ is not and there are as many definitions of $Q$ as there are parton shower models. In Chaps. 2 and 3 we present new methods for constructing the splitting functions which allow for, potentially, much faster evaluation of the Sudakov form factor.

\subsection{Jets}

As discussed earlier, jets are the result of the parton shower process and are ubiquitous in a hadron collider environment. The prediction of jets in QCD and their subsequent observation in experiments performed in the 1970s at DESY, SLAC, and elsewhere was a coup for QCD. At that time, observables such as thrust [5] were proposed that allowed for the qualitative study of jet production. These such observables have the property known as infrared and collinear (IRC) safety. IRC safety is 
the property that observables are insensitive to very soft or collinear radiation. This property is important for the ability to compute the properties of these observables in perturbation theory: only IRC safe observables lead to finite predictions to all orders in perturbation theory. With our current understanding of QCD as a perturbative gauge theory at high energies, IRC safe observables are the only observables that are unambiguously sensible.

Nevertheless, the definition of a jet is ambiguous and studying jets requires a rigorous, algorithmic procedure. Because of the scale-invariant or fractal structure of jets, the distinction between one or two or any number of jets is ill-defined without a definition. The original jet definition due to Sterman and Weinberg [6] defined jets by demanding a large deposit of energy in a small solid angle in the experiment. This jet definition is IRC safe and can be studied and properties of these jets can be predicted in QCD. However, it took many years for much of the community to appreciate the necessity of IRC safety for jet definitions, especially at hadron colliders.

As more was learned about QCD and the properties of jets were becoming more and more well-understood, new observables and jet definitions were proposed. Many now "standard" jet definitions began from the idea that they could be defined to "undo" the parton shower. In particular, in a parton shower, the softest or most collinear radiation is emitted last and so these jet definitions attempt to combine the softest or most collinear radiation at every step so as to reconstruct the particle that originated in the hard interaction. These procedures are referred to as "sequential jet algorithms" and have the following structure:

1. Begin with all measured particles in the detector.

2. Compute the metric $d_{i j}$ between all pairs of particles.

3. If the smallest $d_{i j}$ is larger than a fiducial value $d$, then call all remaining particles jets and exit the algorithm.

4. If the smallest $d_{i j}$ is smaller than $d$, then merge those particles $i$ and $j$ into particle $I$.

5. Add $I$ to the list of particles and remove $i$ and $j$. 


\section{Return to step 2.}

The precise definition of the metric $d_{i j}$ defines the sequential jet algorithm. In particular, there are three popular classes of sequential jet algorithm for hadron colliders as defined by an integer $n$ :

$$
d_{i j}=\min \left\{p_{\perp i}^{n}, p_{\perp i}^{n}\right\} \Delta R_{i j}^{2} .
$$

$p_{\perp i}$ is the transverse momentum as measured with respect to the proton beam axis and $\Delta R_{i j}$ is an angular measure between the momentum of particles $i$ and $j$. The class of algorithm is defined by the power to which the transverse momentum is raised: $n>0$ is the $k_{T}$ algorithm [7], $n=0$ is the Cambridge/Aachen algorithm [8], and $n<0$ is the anti- $k_{T}$ algorithm [9]. The merging procedure in step 4 is typically defined by adding the four-momentum of the particles $i$ and $j$. It is important to note that these jet algorithms are all IRC safe.

Recently, there has been a renewed interest in studying jets in their own right, and specifically, studying the substructure of jets [10 12]. The motivation for this focus is the high energies that are made available by the LHC. Unstable particles can be produced in the hard interaction and typically decay to several jets. If the particles are also produced at high energy, the decay products are boosted and will be combined in a single jet. If the goal of the experiment is to find these unstable particles, for example, the Higgs boson, this is a problem. It was realized that it is possible to determine the true identity of such a jet by studying the distribution of energy in the jet. This program is referred to as "jet substructure".

The jet substructure program has typically focused on algorithms for identifying the particles which initiate a jet. Standard procedures include identifying top quarks, $W$ or $Z$ bosons, or the Higgs boson from a background of jets initiated by QCD. Myriad IRC safe observables or procedures have been introduced, each of which focus on different physics of a jet: some focus on the flow of color in a jet, others the kinematics of the particles in a jet or others study the branching tree as generated by a sequential jet algorithm in its own right. 


\subsection{Outline}

The outline of this thesis is as follows. We begin in Chaps. 2 and 3 by presenting a new procedure for constructing the QCD parton shower. In Chap. 2, we introduce splitting functions that are functions of the spin of the radiating and radiated particles in the parton shower appropriate for massless particles. These splitting functions can be simply computed from basic Feynman diagram calculations in QCD and by defining operators to project onto states of definite spin. Usually in parton showers spins are summed over and so we show that summing over the spin-dependent splitting functions reproduces the standard results. Also, in this chapter is introduced a procedure called sector decomposition for the parton shower.

Chap. 3 continues the work in Chap. 2 by considering the effects of adding masses to particles. At the high energies available at the LHC, it is important to consider the radiation from massive particles such as the top quark when simulating and making predictions. Spin information of a massive particle is not uniquely defined because in the particle's center of mass frame, there is no preferred direction. However, massive particles can have definite chirality, and this is how we define the massive spin-dependent splitting functions. This still leads to the introduction of an arbitrary axis to define spin; however, summing over spins leads to a result that is independent of any external axis. The analysis of this chapter is based on soft and quasi-collinear limits of massive QCD processes, which we also discuss.

While Chaps. 2 and 3 were new methods for modeling the radiation from quarks and gluons, Chap. 4 defines new observables for studying jets and QCD. In [13], along with another graduate student, Martin Jankowiak, I introduced a procedure for identifying structure in jets by defining a correlation function on the constituents of a jet which we called the "angular correlation function". In Chap. 4, the behavior of the angular correlation function is studied in jet ensembles which leads to an observable that has a nice physical interpretation. The average angular correlation function is introduced and defined which allows for a study of the gross or average properties of QCD jets. The angular correlation function directly probes the scaling behavior of a jet and, if QCD jets are scale-invariant, the angular correlation function 
should have simple properties. We discuss and compute the effects of the simplest physics of jets on the form of the angular correlation function and compare the output of popular Monte Carlo generators to one another. In addition to studying jets, we also present a study of radiation that is weakly correlated with the radiation from the hard interaction, coming from ISR or the underlying event. Simple models of the underlying event allow for an analytical study of the angular correlation function, which we present. 


\section{Part I}

\section{New Models for Jet Structure}




\section{Chapter 2}

\section{Spin-Dependent Antenna Splitting Functions}

\subsection{Introduction}

In the studies that are now being done to prepare for physics at the LHC, many new approaches have been proposed to the old problem of generating parton showers. The workhorse event generators PYTHIA [14] and HERWIG [15] generate parton showers by successive radiations from individual partons. The 'splitting functions' that define the radiation pattern are taken to be the kernels in the Altarelli-Parisi equation [16, 17]. This guarantees that the radiation pattern is correct in the region in which two partons become collinear. Marchesini and Webber pointed out that it is also important to include color interference between emissions from different partons [18]. In the workhorse generators, this is implemented by angular ordering of emissions.

The program ARIADNE, by Andersson, Gustafson, Lönnblad, and Pettersson, took a different approach, implementing color coherence by considering the QCD dipole to be the basic object that radiates a parton [19, 20]. The basic branching process in a parton shower is then a splitting in which two partons forming a color dipole radiate a third parton. This approach has been taken up recently by a number of authors. It is the basis for the VINCIA shower by Giele, Kosower, and Skands [21] 
and the parton shower implementation in SHERPA by Krauss and Winter [22]. We are also developing a parton shower based on this approach [23]. In the years between ARIADNE and the newer works, the term 'dipole' has been applied in QCD to a different strategy based on $1 \rightarrow 2$ splittings with recoil taken up by a third particle [24]. To avoid confusion, we will follow [21] in calling the initial two-parton state an 'antenna' and a branching process with $2 \rightarrow 3$ splittings an 'antenna shower'.

Central to the antenna shower is the $2 \rightarrow 3$ splitting function, the function that gives the relative branching probabilities as a function of the final momenta. The original ARIADNE program used an ad hoc proposal satisfying the basic consistency requirements. It would be better to have a prescription that can be directly derived from QCD. Splitting to three partons has been studied in great detail in the QCD literature, but not for this application. Collinear systems of three partons are a part of the infrared structure of QCD at next-to-next-to-leading order, and calculations that reach this level need an explicit prescription for treating this set of infrared singularities. Kosower [25] defined the 'antenna function' as a basic starting point for the analysis of this problem. Many authors have computed antenna functions [26 28]. Quite recently, Gehrmann-De Ridder, Gehrmann, and Glover have built a complete formalism of 'antenna subtraction' for NNLO calculations [29 31]. The kernel in their theory can be interpreted as a $2 \rightarrow 3$ splitting function, and it has been used to perform $2 \rightarrow 3$ splitting in the VINCIA shower [21].

In this paper, we will take a much more direct route to the construction of $2 \rightarrow 3$ splitting functions. We will compute these functions by writing local operators that create two-parton final states and computing their 3-parton matrix elements. These calculations are very straightforward. They can be used to treat individually all possible sets of polarized initial and final partons.

This paper is organized as follows: In Section 2, we will present our complete set of spin-dependent $2 \rightarrow 3$ splitting functions. In Section 3, we will give the derivation for the cases with total spin zero. In Sections 4 and 5, we will give the derivation for the cases with nonzero total spin.

All of these derivations will be done in the kinematics of final-state radiation. This is the easiest situation to visualize and understand. However, the same splitting 
functions can be used, after crossing, to describe parton emissions that involve initialstate particles. We will explain how to use our expressions for initial-state showers in Section 6.

The $1 \rightarrow 2$ Altarelli-Parisi splitting functions are universal in the sense that they result from a well-defined singular limit of QCD amplitudes. For $2 \rightarrow 3$ splitting functions there is no such universality. The collinear and soft limits must agree with the known universal values, but away from these limits there is no unique prescription. Earlier in this introduction, we made reference to a number of previous proposals for the spin-averaged antenna splitting functions. All of these, including the ARIADNE splitting functions, have the correct soft and collinear limits and so satisfy the basic requirements. In Section 7 , we will give a detailed comparison of the $2 \rightarrow 3$ splitting functions obtained using our method to previous proposals for these splitting functions.

\subsection{Proposal for the $2 \rightarrow 3$ splitting functions}

We begin by defining variables for $2 \rightarrow 3$ splitting. There are three cases of splittings that are needed for antenna showers: the final-final (FF) splitting, in which a third particle is created by coherent radiation from a two-particle system in the final state; the initial-final (IF) splitting, in which a third particle is created by coherent radiation from an initial- and a final-state particle; and initial-initial (II) splitting, in which a third particle is created by coherent radiation from two initial-state particles. It is easiest to understand the kinematics of antenna splitting for the FF case. In this section, we will explain this kinematics and give a precise prescription for the splitting functions. In Section 6, we will extend our prescription to the IF and II cases, in such a way that the same splitting functions can be used in those cases.

Consider, then, a two-parton final-state system $(A, B)$ that splits to a 3-parton system $(a, c, b)$, conserving momentum, as shown in Fig. 2.1(a). Let $s_{i j}=\left(k_{i}+k_{j}\right)^{2}$, and let $Q=k_{A}+k_{B}=k_{a}+k_{b}+k_{c}$. 


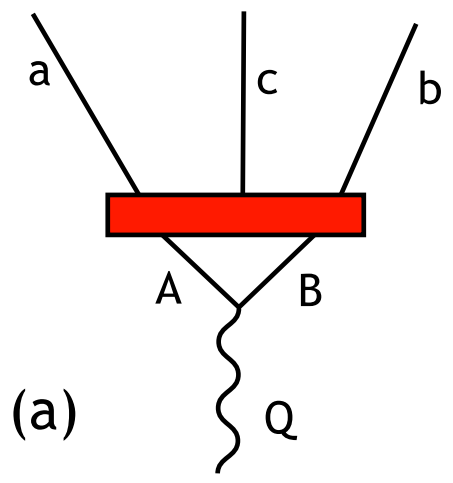

(b)

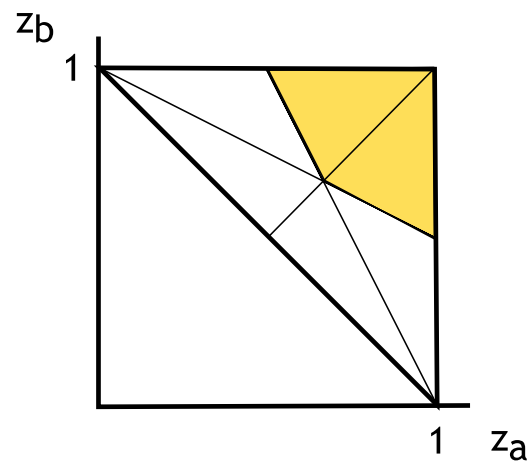

Figure 2.1: (a) Kinematics of $2 \rightarrow 3$ splitting in the final state (FF) case. (b) Phase space for $2 \rightarrow 3$ splitting in the FF case. The six regions corresponding to different orderings of $s_{a b}, s_{a c}, s_{b c}$ are shown. The region that should be well described by an antenna splitting $A B \rightarrow a c b$ is shaded.

The fractional invariant masses in the final state are

$$
y_{a b}=\frac{s_{a b}}{s_{A B}}, \quad y_{a c}=\frac{s_{a c}}{s_{A B}}, \quad y_{b c}=\frac{s_{b c}}{s_{A B}} .
$$

The momentum fractions of the three particles in the $(A B)$ frame are

$$
z_{a}=\frac{2 Q \cdot k_{a}}{s_{A B}}, \quad z_{b}=\frac{2 Q \cdot k_{b}}{s_{A B}}, \quad z_{c}=\frac{2 Q \cdot k_{c}}{s_{A B}} .
$$

These obey the identities

$$
y_{a b}=\left(1-z_{c}\right), \quad y_{a c}=\left(1-z_{b}\right), \quad y_{b c}=\left(1-z_{a}\right)
$$

and

$$
y_{a b}+y_{a c}+y_{b c}=1, \quad z_{a}+z_{b}+z_{c}=2 .
$$

The FF phase space covers the triangle $z_{a} \leq 1, z_{b} \leq 1, z_{a}+z_{b} \geq 1$. We can divide this phase space into six triangles, each of which has a different ordering of the three quantities $y_{a b}, y_{a c}, y_{b c}$, as shown in Fig. 2.1(b). An antenna shower should give an accurate description of the dynamics in the two regions $y_{a c}<y_{b c}<y_{a b}, y_{b c}<y_{a c}<y_{a b}$ that are shaded in the figure. 
A general problem in the generation of QCD radiation is that of possible doublecounting. Consider, for example, the process $e^{+} e^{-} \rightarrow q g g \bar{q}$. In some part of the phase space, the first $g$ can be considered to be radiated from the antenna of the $q$ and the second $g$; in another, the second $g$ can be considered to be radiated from the first $g$ and the $\bar{q}$. These regions should be disjoint in the full 4-body phase space. The complete solution to the problem is beyond the scope of this paper. In simple terms, though, we can make the separation by choosing the radiated gluon to be softer than the gluon from which it radiates. This corresponds to integrating each antenna only over the shaded region in Fig. 2.1(b). A similar approximate solution to the doublecounting problem will apply in the other kinematic regions discussed in Section 6. A more detailed discussion of this issue can be found in [21, 23].

Radiation from different QCD antenna is strictly independent and non-interfering only in the limit of a large number of colors in QCD, $N_{c} \gg 1$. Keeping only terms leading in $N_{c}$ is known to be a good approximation to full QCD in many circumstances. In particular, parton shower algorithms are correct only to leading order in $N_{c}$. In this paper, we will explicitly work only to the leading order for large $N_{c}$.

In the limit of large $N_{c}$, the rate for a $2 \rightarrow 3$ splitting is given by a formula of the form

$$
N_{c} \frac{\alpha_{s}}{4 \pi} \int d z_{a} d z_{b} \cdot \mathcal{S}\left(z_{a}, z_{b}, z_{c}\right)
$$

For example, in $e^{+} e^{-} \rightarrow q_{-} g_{+} \bar{q}_{+}$,

$$
\frac{1}{\sigma_{0}} \frac{d \sigma}{d z_{a} d z_{b}}=N_{c} \frac{\alpha_{s}}{4 \pi} \frac{z_{a}^{2}}{\left(1-z_{a}\right)\left(1-z_{b}\right)},
$$

where $(a, c, b)$ are the $(q, g, \bar{q})$, respectively, - and + denote left- and right-handed helicity, and $\sigma_{0}$ is the cross section for $e^{+} e^{-} \rightarrow q_{-} \bar{q}_{+}$. Eq. 2.5 will be our basic formula of reference. Using this notation, we can write the various $2 \rightarrow 3$ splitting

\footnotetext{
${ }^{1}$ In writing a parton shower for $e^{+} e^{-} \rightarrow q \bar{q}$, one might pick up an obvious $1 / N_{c}$ correction by writing the prefactor as $\frac{8}{3}$ instead of 3 for the first gluon emission from the quark line. In higher orders, the analogous treatment of radiation from quark lines is not so innocent and can potentially lead to incorrect accounting of color coherence.
} 
functions as

$$
\mathcal{S}=\frac{\mathcal{N}\left(z_{a}, z_{b}, z_{c}\right)}{y_{a b} y_{a c} y_{b c}}
$$

where the numerator is a simple function of the $z_{i}$. For example, for the splitting $q_{-} \bar{q}_{+} \rightarrow q_{-} g_{+} \bar{q}_{+}$given above,

$$
\mathcal{N}=y_{a b} z_{a}^{2}=\left(1-z_{c}\right) z_{a}^{2}
$$

In Table 2.1, we give our proposal for the numerator functions for all possible cases of massless quark and gluon splittings. The expressions are all monomials in the $y_{i j}$ and $z_{j}$.

In the FF kinematics, all of the $y_{i j}$ and $z_{i}$ are positive and so $\mathcal{S}\left(z_{a}, z_{b}, z_{c}\right)$ in (2.7), is always positive, In IF and II kinematics, some $y_{i j}$ and $z_{i}$ will become negative. In most cases, the correct prescription is to take $\mathcal{S}\left(z_{a}, z_{b}, z_{c}\right)$ to be the absolute value of the expression in Table 2.1. However, there is a line within the IF region where $z_{a}$ or $z_{b}$ crosses from positive to negative values. A few entries in the Table change sign across this line. We recommend that those entries be set to zero when $z_{a}$ or $z_{b}$ are negative. We will give a detailed discussion of these points in Section 6 .

The splitting functions $\mathcal{S}$ must give the correct universal behavior in the soft and collinear limits. In the soft limit, $z_{c} \rightarrow 0$, the numerators must go to 1 if the flavor and helicity of the final partons $a$ and $b$ match those of the initial partons $A$ and $B$; otherwise, the numerators must go to 0 . It is easy to check that this test is satisfied.

In the collinear limits, we will insist that each antenna has the collinear behavior required in QCD. One often hears the following statement about soft and collinear limits: In dipole splitting ( $\rightarrow 2$ emission), each dipole has the correct collinear behavior but the correct soft behavior is obtained by combining neighboring dipoles. In antenna splitting ( $2 \rightarrow 3$ emission), each antenna has the correct soft limit but the correct collinear behavior is obtained by combining neighboring antennae. However, in the large $N_{c}$ limit, which we take to guide our intuition, different antennae are independent radiators with different, non-interfering, colors flowing in them. From the viewpoint of this limit, each antenna, separately, must give both the correct pattern of soft radiation and the correct pattern of collinear radiation. This philosophy differs 


\begin{tabular}{c|cccccccc} 
& +++ & ++- & +-+ & -++ & --+ & -+- & +-- & --- \\
\hline$g_{+} g_{+} \rightarrow g g g$ & 1 & $y_{a c}^{4}$ & $y_{a b}^{4}$ & $y_{b c}^{4}$ & 0 & 0 & 0 & 0 \\
$g_{-} g_{+} \rightarrow g g g$ & 0 & 0 & $y_{b c}^{4}$ & $z_{a}^{4}$ & $z_{b}^{4}$ & $y_{a c}^{4}$ & 0 & 0 \\
$g_{+} g_{+} \rightarrow \bar{q} q g$ & - & - & $y_{a b}^{3} y_{b c}$ & $y_{a b} y_{b c}^{3}$ & - & 0 & 0 & - \\
$g_{-} g_{+} \rightarrow \bar{q} q g$ & - & - & $y_{a b} y_{b c}^{3} z_{b}^{2}$ & $z_{a}^{2} z_{b}^{2} y_{a b} y_{b c}$ & - & 0 & 0 & - \\
$q_{-} \bar{q}_{+} \rightarrow q g \bar{q}$ & - & - & - & $y_{a b} z_{a}^{2}$ & $y_{a b} z_{b}^{2}$ & - & - & - \\
$q_{-} \bar{q}-\rightarrow q g \bar{q}$ & - & - & - & - & - & $y_{a b}^{3}$ & - & $y_{a b}$ \\
$q_{-} g_{-} \rightarrow q g g$ & - & - & - & 0 & $y_{a c}^{4}$ & $y_{a b}^{3} z_{b}$ & - & $z_{a}$ \\
$q_{-} g_{+} \rightarrow q g g$ & - & - & - & $z_{a}^{3}$ & $y_{a b} z_{b}^{3}$ & $y_{a c}^{4}$ & - & 0 \\
$q_{-} g_{-} \rightarrow q \bar{q} q$ & - & - & - & - & $y_{a b} y_{a c}^{3}$ & $y_{a b}^{2} y_{a c} z_{b}$ & - & - \\
$q_{-} g_{+} \rightarrow q \bar{q} q$ & - & - & - & - & $z_{a} y_{a b} y_{a c} z_{b}^{2}$ & $z_{a} y_{a b} y_{a c}^{3}$ & - & -
\end{tabular}

Table 2.1: Numerator functions $\mathcal{N}\left(z_{a}, z_{b}, z_{c}\right)$ for the spin-dependent $2 \rightarrow 3$ splitting functions $A B \rightarrow a c b: \mathcal{S}=\mathcal{N} /\left(y_{a b} y_{a c} y_{b c}\right)$. Each line gives a choice of $A B$. The labels denote the polarization of the three final particles with the radiated particle $c$ in the center: $\left(h_{a}, h_{c}, h_{b}\right)$. The empty columns are forbidden by quark chiral symmetry. By the $\mathrm{P}$ and $\mathrm{C}$ invariance of QCD, the same expressions apply after exchanging $-\leftrightarrow+, q \leftrightarrow \bar{q}$, or $A B a c b \leftrightarrow B A b c a$.

from that of the ARIADNE group [19, 20] and of [22]. We will discuss this point further when we compare with their results in Section 7.

The collinear radiation from a given hard gluon is then the sum of two contributions, one from each of the two antennae to which that hard gluon belongs. In the large $N_{c}$ limit, these correspond to radiation from the color and anticolor lines of the gluon. A single antenna, which has one of these contributions, then has $\frac{1}{2}$ of the standard collinear emission rate. This factor of $\frac{1}{2}$ enters the check will we perform in a moment. The factor comes entirely from bookkeeping and is independent of the question of double-counting discussed briefly earlier in this Section.

We now discuss the check of collinear limits. Consider the limit in which $c$ becomes collinear with $a$. In this limit,

$$
z_{c} \rightarrow z, \quad z_{a} \rightarrow(1-z), \quad z_{b} \rightarrow 1, \quad y_{a c} \rightarrow 0
$$

The $2 \rightarrow 3$ splitting function must reduce to

$$
\mathcal{S} \rightarrow \frac{1}{y_{a c}} P(z),
$$




\begin{tabular}{c|cccc} 
& ++ & -+ & +- & -- \\
\hline$g_{+} \rightarrow g g:$ & $1 / z(1-z)$ & $(1-z)^{3} / z$ & $z^{3} /(1-z)$ & 0 \\
$g_{+} \rightarrow q \bar{q}:$ & - & $(1-z)^{2}$ & $z^{2}$ & - \\
$q_{-} \rightarrow g q:$ & - & - & $(1-z)^{2} / z$ & $1 / z$ \\
$q_{-} \rightarrow q g:$ & - & $z^{2} /(1-z)$ & - & $1 /(1-z)$
\end{tabular}

Table 2.2: Spin-dependent Altarelli-Parisi splitting functions $P(z)$ for splittings $B \rightarrow c b$. The labels denote the polarization of the two final particles with the radiated particle first: $\left(h_{c}, h_{b}\right)$. The empty columns are forbidden by quark chiral symmetry. By the $\mathrm{P}$ and $\mathrm{C}$ invariance of QCD, the same expressions apply after exchanging $-\leftrightarrow+$ or $q \leftrightarrow \bar{q}$.

here $P(z)$ is the relevant spin-dependent Altarelli-Parisi splitting function. These were presented in the original Altarelli-Parisi paper [16] and are reviewed in Table 2.2. The functions are normalized as in (2.5), and as described in the previous paragraph: We take the large $N_{c}$ limit and divide by 2 where necessary to give the contribution from one QCD antenna. The denominator of 2.7$)$ tends to $y_{a c} z(1-z)$ in this limit. Then it is easy to check that the numerators match correctly in all cases. The limit in which $c$ becomes collinear with $b$ can be checked in the same way.

When the collinear limits and the soft limit are all nonzero, there is a unique monomial of the $y$ 's and $z$ 's that gives all limits correctly. In the other cases, there is some ambiguity. In all cases, it would be desirable if the results in Table 2.1 could be derived directly by simple Feynman diagram computations. In the next few sections, we will present those derivations.

\section{$2.3 \quad$ Spin-0 case}

To compute the $2 \rightarrow 3$ splitting functions, we will use the following method: Write an operator that, at the leading order, creates a 2-parton state with definite helicity. Then, compute the 3-particle matrix element. This realizes in a very simple way the splitting process illustrated in Fig. 2.1.

To create massless quarks and antiquarks of definite helicity, we will use the appropriate chiral fermion fields. To create gluons of definite helicity, we will use the 
operators

$$
\sigma \cdot F=\frac{1}{2} \sigma^{m} \bar{\sigma}^{n} F_{m n}, \quad \bar{\sigma} \cdot F=\frac{1}{2} \bar{\sigma}^{m} \sigma^{n} F_{m n}
$$

where $\sigma^{m}, \bar{\sigma}^{m}$ are the $2 \times 2$ matrix entries of the Dirac matrices in a chiral basis and $F_{m n}$ is the gluon field strength tensor. At leading order, $\sigma \cdot F$ creates a + helicity gluon, and $\bar{\sigma} \cdot F$ creates a - helicity gluon.

The 2-parton state $g_{+} g_{+}$in the first line of Table 2.1 can be created from the spin-0 operator

$$
\mathcal{O}=\frac{1}{2} \operatorname{tr}\left[(\sigma \cdot F)^{2}\right]
$$

We can then compute the splitting function for this polarized initial state explicitly from the definition

$$
\mathcal{S}\left(z_{a}, z_{c}, z_{b}\right)=Q^{2}\left|\frac{\mathcal{M}(\mathcal{O} \rightarrow a c b)}{\mathcal{M}(\mathcal{O} \rightarrow A B)}\right|^{2}
$$

In the next few sections, we will compute all of the splitting functions in Table 2.1 using this formula, with a different choice of the operator $\mathcal{O}$ for each line of the table.

To evaluate (2.13), we need to compute the matrix elements of $\mathcal{O}$, with total momentum $Q$ injected, to 3-gluon final states. The result can be expressed in terms of color-ordered amplitudes. We identify the color-ordered amplitude that multiplies the color structure $\operatorname{tr}\left[T^{a} T^{c} T^{b}\right]$ with the splitting function. To carry out these computations, we will use the spinor product formalism. That is, instead of working with 4 -vectors, we will use as our basic objects the spinor products

$$
\langle i j\rangle=\bar{u}_{-}(i) u_{+}(j), \quad[i j]=\bar{u}_{+}(i) u_{-}(j)
$$

These objects obey

$$
|\langle i j\rangle|^{2}=|[i j]|^{2}=s_{i j}
$$

Methods for QCD computations with spinor products and color-ordering are explained in [32, 33]. In this notation, the matrix element for $\mathcal{O}$ to create a $g_{+} g_{+}$ final state is

$$
\left\langle g_{+} g_{+}|\mathcal{O}| 0\right\rangle=[A B]^{2}
$$

The three-gluon matrix elements of the operator (2.12) are given by the diagrams 


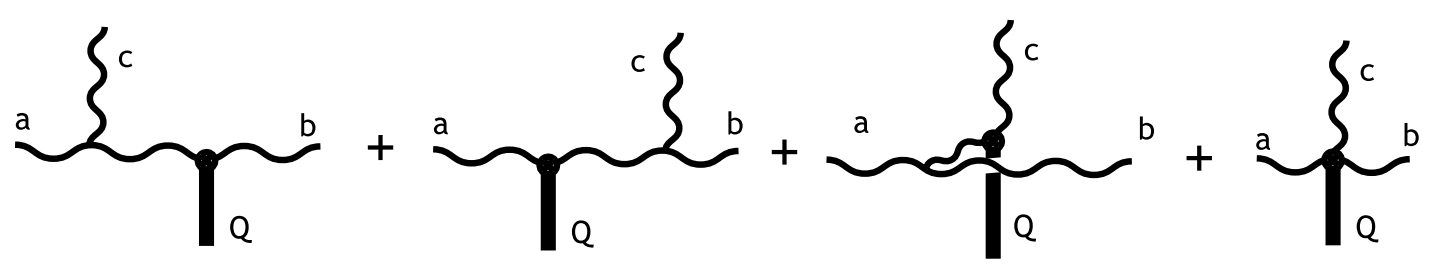

Figure 2.2: Feynman diagrams for the computation of the $g g \rightarrow g g g$ splitting functions.

in Fig. 2.2. These diagrams have already been analyzed by Dixon, Glover, and Khoze as a part of their analysis of the coupling of the Higgs boson to multi-gluon states [34]. They find

$$
\begin{aligned}
& \mathcal{A}\left(\mathcal{O} \rightarrow g_{+} g_{+} g_{+}\right)=\frac{s_{A B}^{2}}{\langle a c\rangle\langle c b\rangle\langle b a\rangle} \\
& \mathcal{A}\left(\mathcal{O} \rightarrow g_{+} g_{+} g_{-}\right)=\frac{[a c]^{4}}{[a c][c b][b a]} \\
& \mathcal{A}\left(\mathcal{O} \rightarrow g_{+} g_{-} g_{+}\right)=\frac{[a b]^{4}}{[a c][c b][b a]} \\
& \mathcal{A}\left(\mathcal{O} \rightarrow g_{-} g_{+} g_{+}\right)=\frac{[b c]^{4}}{[a c][c b][b a]}
\end{aligned}
$$

and zero for the other four cases. After squaring, using (2.15), and dividing by the square of (2.16), we obtain the first line of Table 2.1.

One of the major points of [34] is that the results (2.17) belong to series of Maximally Helicity Violating (MHV) amplitudes that have a simple form for any number of gluons emitted. Actually, all of the amplitudes that we will compute in this paper are similarly simple and belong to MHV series. The use of MHV amplitudes to study antenna splitting is explored for higher-order processes in [28].

In principle, the initial state $g_{+} g_{+}$could also have been created by an operator of spin 2, or some higher spin. This would have led to a more complicated expression for the $2 \rightarrow 3$ splitting function, with, however, the same soft and collinear limits. This illustrates the ambiguity in the definitions of $2 \rightarrow 3$ splitting functions refered to 


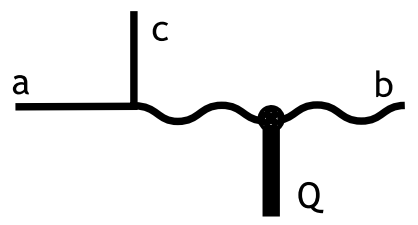

Figure 2.3: Feynman diagram for the computation of the $g g \rightarrow \bar{q} q g$ splitting functions.

in the introduction. The simplest results are obtained using the operator of minimal spin, and we will make that choice in all of the examples to follow.

The diagram shown in Fig. 2.3 gives the splitting of the two-gluon initial state to $\bar{q} q g$. We find

$$
\begin{aligned}
& \mathcal{A}\left(\mathcal{O} \rightarrow \bar{q}_{+} q_{-} g_{+}\right)=\frac{[a b]^{2}}{[a c]} \\
& \mathcal{A}\left(\mathcal{O} \rightarrow \bar{q}_{-} q_{+} g_{+}\right)=\frac{[c b]^{2}}{[a c]}
\end{aligned}
$$

There is no splitting to a final $g_{-}$. This gives the result in the third line of the table.

The initial state $q_{-} \bar{q}_{-}$can also be created by a spin 0 operator

$$
\mathcal{O}=\bar{q}_{L} q_{R}
$$

The matrix element for this operator to create a $q_{-} \bar{q}_{-}$final state is

$$
\left\langle q_{-} \bar{q}_{-}|\mathcal{O}| 0\right\rangle=\langle A B\rangle
$$

A straightforward calculation gives

$$
\begin{aligned}
& \mathcal{A}\left(\mathcal{O} \rightarrow q_{-} g_{+} \bar{q}_{-}\right)=\frac{\langle a b\rangle^{2}}{\langle a c\rangle\langle c b\rangle} \\
& \mathcal{A}\left(\mathcal{O} \rightarrow q_{-} g_{-} \bar{q}_{-}\right)=\frac{s_{A B}}{[a c][c b]}
\end{aligned}
$$

These give the results shown in the sixth line of the table. 


\subsection{Spin-1 and spin-2 case}

In [19], the $2 \rightarrow 3$ splitting function for $q \bar{q} \rightarrow q g \bar{q}$ was derived from the cross section for $e^{+} e^{-} \rightarrow q q \bar{q}$. From the point of view of the previous section, this corresponds to creating the 2- and 3-parton final states using the operator

$$
\mathcal{O}=\bar{q}_{L} \gamma^{m} q_{L}
$$

To obtain a definite matrix element, we must contract this operator with a polarization vector. A convenient choice is to introduce two new massless vectors 1 and 2, such that $k_{1}+k_{2}=k_{A}+k_{B}$, and to choose the polarization vector to be $\epsilon^{\mu}=\left\langle 1\left|\gamma^{\mu}\right| 2\right]$. This is effectively the procedure of decaying the massive vector that couples to the

operator (2.22) into a pair of massless vectors to facilitate the analysis; this is a standard method in spinor product calculations [35. We then recast

$$
\mathcal{O}=\frac{1}{2} \bar{q}_{L} \gamma^{m} q_{L}\left\langle 1\left|\gamma_{m}\right| 2\right] .
$$

The matrix element of $(2.23)$ to a $q_{-} \bar{q}_{+}$state is

$$
\left\langle q_{-} \bar{q}_{+}|\mathcal{O}| 0\right\rangle=-\langle 1 A\rangle[2 B] .
$$

The direction of the 1-2 system chooses the helicity of the final partons. In this case, there is only one choice, and so the amplitude vanishes when 1 is parallel to $A$ or 2 is parallel to $B$. This will not always be true in our later examples. But, we will always be able to choose the desired helicity of $A$ and $B$ by choosing 1 parallel to $B$ and 2 parallel to $A$.

The matrix elements for the operator (2.23) to create 3-parton final states are

$$
\begin{aligned}
& \mathcal{A}\left(\mathcal{O} \rightarrow q_{-} g_{+} \bar{q}_{+}\right)=\frac{\langle 1 a\rangle^{2}[12]}{\langle a c\rangle\langle c b\rangle} \\
& \mathcal{A}\left(\mathcal{O} \rightarrow q_{-} g_{-} \bar{q}_{+}\right)=\frac{[2 b]^{2}\langle 12\rangle}{[a c][c b]} .
\end{aligned}
$$


To compute the results in the fifth line of the table, we must essentially divide (2.25) by (2.24) and square the result. To do this, we need a prescription for treating the expressions $\langle 1 a\rangle$ and $[2 b]$ in the numerators. The problem of relating the vectors $a, b, c$ to $A$ and $B$ in an antenna splitting was discussed at length by Kosower in [36]; that paper gives a general treatment in terms of reconstruction functions to provide expressions that can be smoothly integrated in higher-order QCD calculations. This discussion is generalized to the initial-state channels in [37]. Here, we will take a more ad hoc approach that leads to the simplest formulae with correct singular limits.

Formulae for $\langle 1 a\rangle$ and $[2 b]$ that are simple and become exact in the collinear and soft limits are found by approximating $a$ collinear with $A$ and $b$ collinear with $B$. Then identifying 1 with $B$ and 2 with $A$ gives

$$
|\langle 1 a\rangle|^{2}=s_{B a} \rightarrow z_{a} s_{A B}, \quad|\langle 1 b\rangle|^{2} \rightarrow 0, \quad|\langle 2 a\rangle|^{2} \rightarrow 0,|\langle 2 b\rangle|^{2}=s_{A b} \rightarrow z_{b} s_{A B}
$$

and similarly for the conjugate products. Using this prescription, one obtains the fifth line of the table. This is a more formal version of the argument for these entries already given in Section 2.

In our calculations, we will encounter two more numerator objects that require reconstruction, namely, $\langle 1 c\rangle$ and $\langle 2 c\rangle$. The prescription above gives

$$
|\langle 1 c\rangle|^{2}=s_{B c} \rightarrow\left(y_{b c} / z_{b}\right) s_{A B}, \quad|\langle 2 c\rangle|^{2}=s_{A c} \rightarrow\left(y_{a c} / z_{a}\right) s_{A B}
$$

However, it is potentially dangerous to write factors of $z_{a}, z_{b}$ in the denominator. We will see in Section 6 that such factors would create unphysical singularities when continued to the IF kinematics. These unphysical singularities are avoided in the general formalism used in [36], but at the price of introducing much more complicated formulae. Fortunately, we will see that $\langle 1 c\rangle$ arises only in situations where there is no collinear singularity with $c$ parallel to $b$. In such cases, the remaining universal singular terms - the collinear singularity with $c$ parallel to $a$ and the soft singularitycorrespond to kinematic limits with $z_{b} \rightarrow 1$. A similar consideration applies to $\langle 2 c\rangle$. 
Thus, we choose, instead of using (2.27), to evaluate these quantities as

$$
|\langle 1 c\rangle|^{2}=s_{B c} \rightarrow y_{b c} s_{A B}, \quad|\langle 2 c\rangle|^{2}=s_{A c} \rightarrow y_{a c} s_{A B} .
$$

This gives an incorrect shape in a region where $a$ and $b$ are collinear, but, hopefully, we will not use the $A B \rightarrow a c b$ splitting function to evaluate the rate to fill this region of phase space.

Another choice for evaluating $\langle 1 c\rangle$ and $\langle 2 c\rangle$ is to replace both expressions by $z_{c}$. However, the spinor product $\langle 1 c\rangle$ vanishes in the $b c$ collinear limit but not in the $a c$ collinear limit, and conversely for $\langle 2 c\rangle$, so this choice does not give the universal singularities correctly.

We now apply this formalism to compute the second and fourth lines of Table 2.1 . associated with the $g_{-} g_{+}$antenna. This antenna is created by the spin-2 operator $\operatorname{tr}\left[\gamma^{m}(\bar{\sigma} \cdot F) \gamma^{n}(\sigma \cdot F)\right]$. To make a definite calculation, we need a spin-2 polarization vector. An appropriate choice can be found by introducing the massless vectors 1 and 2 as above and writing

$$
\epsilon^{m n}=\left\langle 1\left|\gamma^{m}\right| 2\right]\left\langle 1\left|\gamma^{n}\right| 2\right] .
$$

This effectively decays the masive spin-2 particle into two massless spinors. This method was introduced in 38 to compute the relevant amplitudes for the emission of massive gravitons at high-energy colliders.

With this prescription, we generate the $g_{-} g_{+}$antenna using the operator

$$
\mathcal{O}=\frac{1}{4} \operatorname{tr}\left[\gamma^{m}(\bar{\sigma} \cdot F) \gamma^{n}(\sigma \cdot F)\right]\left\langle 1\left|\gamma_{m}\right| 2\right]\left\langle 1\left|\gamma_{n}\right| 2\right]
$$

The matrix element of this operator that creates the 2-parton dipole is

$$
\left\langle g_{-} g_{+}|\mathcal{O}| 0\right\rangle=\langle 1 A\rangle^{2}[2 B]^{2} .
$$

To obtain the correct initial polarizations, we take $1=B, 2=A$ as before. The 


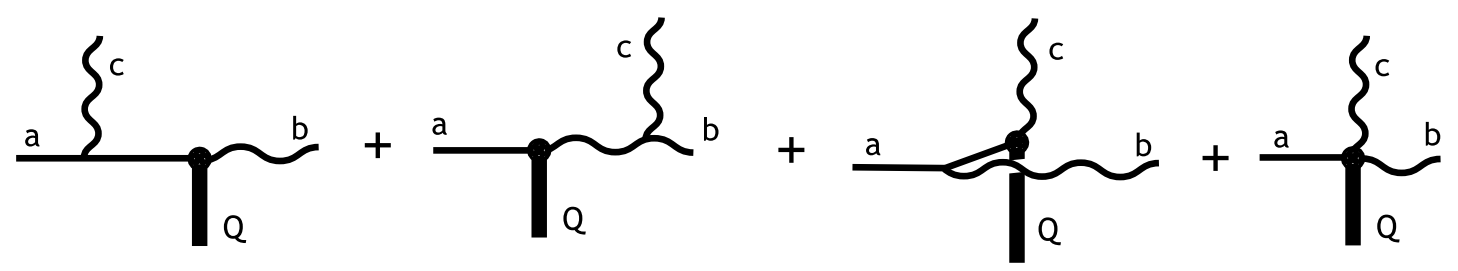

Figure 2.4: Feynman diagrams for the computation of the $q g \rightarrow q g g$ splitting functions.

matrix elements to the possible 3-parton final states are

$$
\begin{aligned}
& \mathcal{A}\left(\mathcal{O} \rightarrow g_{+} g_{+} g_{+}\right)=0 \\
& \mathcal{A}\left(\mathcal{O} \rightarrow g_{+} g_{+} g_{-}\right)=\frac{\langle 1 b\rangle^{4}[12]^{2}}{\langle a b\rangle\langle a c\rangle\langle c b\rangle} \\
& \mathcal{A}\left(\mathcal{O} \rightarrow g_{+} g_{-} g_{+}\right)=\frac{\langle 1 c\rangle^{4}[12]^{2}}{\langle a b\rangle\langle a c\rangle\langle c b\rangle} \\
& \mathcal{A}\left(\mathcal{O} \rightarrow g_{-} g_{+} g_{+}\right)=\frac{\langle 1 a\rangle^{4}[12]^{2}}{\langle a b\rangle\langle a c\rangle\langle c b\rangle},
\end{aligned}
$$

and the conjugates with $1 \leftrightarrow 2$ for the other four combinations. Applying the reductions (2.26), 2.27), we find the results given in the second line of the table.

The nonzero matrix elements of this operator to $\bar{q} q g$ final states are

$$
\begin{aligned}
\mathcal{A}\left(\mathcal{O} \rightarrow \bar{q}_{+} q_{-} g_{+}\right) & =\frac{\langle 1 c\rangle^{2}[2 b]^{2}}{[a c]} \\
\mathcal{A}\left(\mathcal{O} \rightarrow \bar{q}_{-} q_{+} g_{+}\right) & =\frac{\langle 1 a\rangle^{2}[2 b]^{2}}{[a c]} .
\end{aligned}
$$

The same reduction process gives the results in the fourth line of the table.

\subsection{Spin- $\frac{1}{2}$ and spin- $\frac{3}{2}$ cases}

The cases of quark-gluon antennae can be treated in the same way. There is one additional subtlety. In QCD, quarks are color triplets and gluons are color octets, so a quark-gluon operator carries net color. This means that the matrix element for 
gluon emission from a quark-gluon operator is not gauge-invariant unless we allow the gluon also to be emitted from the initial state. This makes it unclear how to define a quark-gluon antenna.

We resolve this problem with the following prescription: We consider the quarks to be color octet particles like the gluons. Then, as in the previous sections, we extract the color-ordered contribution corresponding to emission from the antenna. In the limit of large $N_{c}$, the various antennae in a process radiate independently. The diagrams contributing to a quark-gluon antenna in this prescription are shown in Fig. 2.4. The third diagram, with an intermediate quark line, does not appear in QCD. However, it does nicely provide the missing piece that makes this sum of diagrams gauge-invariant without radiation from the initial state.

This solution is the same as that found in the earlier work of Gehrmann-De Ridder, Gehrmann, and Glover 29 31]. Those authors computed the quark-gluon antennae by factorizing the amplitudes for the decay of a neutralino into a massless gluino plus $g g$ or $q \bar{q}$. In their calculation, the off-shell color octet fermion is the gluino.

With this understanding, we proceed as in the previous Section. We can generate the $q_{-} g_{-}$antenna using the operator $\bar{q}_{L}(\bar{\sigma} \cdot F)$. The polarization spinor can be built by introducing massless spinors 1 and 2 as above and taking $|2\rangle$ to be this spinor. Then

$$
\mathcal{O}=-i \bar{q}_{L}(\bar{\sigma} \cdot F)|2\rangle
$$

The matrix element of this operator that creates the 2-parton dipole is

$$
\left\langle q_{-} g_{-}|\mathcal{O}| 0\right\rangle=\langle A B\rangle[B 2]
$$

To obtain the correct initial polarizations, we take $1=B, 2=A$.

The matrix elements to the possible 3-parton final states are

$$
\begin{aligned}
& \mathcal{A}\left(\mathcal{O} \rightarrow q_{-} g_{+} g_{+}\right)=0 \\
& \mathcal{A}\left(\mathcal{O} \rightarrow q_{-} g_{-} g_{+}\right)=\frac{\langle a c\rangle^{3}\langle 2 c\rangle}{\langle a b\rangle\langle a c\rangle\langle c b\rangle} \\
& \mathcal{A}\left(\mathcal{O} \rightarrow q_{-} g_{+} g_{-}\right)=\frac{\langle a b\rangle^{3}\langle 2 b\rangle}{\langle a b\rangle\langle a c\rangle\langle c b\rangle}
\end{aligned}
$$




$$
\mathcal{A}\left(\mathcal{O} \rightarrow q_{-} g_{-} g_{-}\right)=\frac{s_{A B}\langle 12\rangle[1 a]}{[a b][a c][c b]}
$$

Applying the reductions (2.26), 2.27), we find the results given in the seventh line of the table.

The nonzero matrix elements of this operator to $q \bar{q} q$ final states are

$$
\begin{aligned}
& \mathcal{A}\left(\mathcal{O} \rightarrow q_{-} \bar{q}_{-} q_{+}\right)=\frac{\langle a c\rangle\langle 2 c\rangle}{\langle c b\rangle} \\
& \mathcal{A}\left(\mathcal{O} \rightarrow q_{-} \bar{q}_{+} q_{-}\right)=-\frac{\langle a b\rangle\langle 2 b\rangle}{\langle c b\rangle} .
\end{aligned}
$$

The same reduction process gives the results in the ninth line of the table.

We generate the $q_{-} g_{+}$antenna using the spin- $\frac{3}{2}$ operator $\bar{q}_{L} \gamma^{m}(\sigma \cdot F)$. This is essentially the supersymmetry current of the system of gluons and color octet fermions. The polarization spinor can be built by introducing massless spinors 1 and 2 as above:

$$
\left.\mathcal{O}=i \bar{q}_{L} \gamma^{m}(\sigma \cdot F) 2\right]\left\langle 1\left|\gamma_{m}\right| 2\right]
$$

The matrix element of this operator that creates the 2-parton dipole is

$$
\left\langle q_{-} g_{+}|\mathcal{O}| 0\right\rangle=\langle 1 A\rangle[2 B]^{2}
$$

To obtain the correct initial polarizations, we again take $1=B, 2=A$.

The matrix elements to the possible 3-parton final states are

$$
\begin{aligned}
& \mathcal{A}\left(\mathcal{O} \rightarrow q_{-} g_{+} g_{+}\right)=\frac{\langle 1 a\rangle^{3}[12]^{2}}{\langle a b\rangle\langle a c\rangle\langle c b\rangle} \\
& \mathcal{A}\left(\mathcal{O} \rightarrow q_{-} g_{-} g_{+}\right)=\frac{\langle a b\rangle[2 b]^{3}\langle 12\rangle}{[a b][a c][c b]} \\
& \mathcal{A}\left(\mathcal{O} \rightarrow q_{-} g_{+} g_{-}\right)=\frac{\langle a c\rangle[2 c]^{3}\langle 12\rangle}{[a b][a c][c b]} \\
& \mathcal{A}\left(\mathcal{O} \rightarrow q_{-} g_{-} g_{-}\right)=0 .
\end{aligned}
$$

Applying the reductions $(2.26), 2.27)$, we find the results given in the eighth line of 
the table.

The nonzero matrix elements of this operator to $q \bar{q} q$ final states are

$$
\begin{aligned}
& \mathcal{A}\left(\mathcal{O} \rightarrow q_{-} \bar{q}_{-} q_{+}\right)=\frac{\langle 1 a\rangle[2 b]^{2}}{[c b]} \\
& \mathcal{A}\left(\mathcal{O} \rightarrow q_{-} \bar{q}_{+} q_{-}\right)=-\frac{\langle 1 a\rangle[2 c]^{2}}{[c b]} .
\end{aligned}
$$

The same reduction process gives the results in the tenth line of the table.

\subsection{Initial-state showers}

The Feynman diagram computations that we have done to find the antenna splitting functions for FF splittings can also be applied, by crossing, to IF and II splittings. The expressions in Table 2.1 are given in terms of invariant quantities that are unchanged under crossing. Thus, we can use the expressions in this table directly in other channels. At worst, a change of the overall sign is required in some cases. In this section, we will clarify this statement by analyzing the kinematics of IF and II splittings in the same variables as those used in Section 2 for FF splittings. In all cases, the kinematics is done for all massless partons only. The kinematic discussion in this section is similar to that presented in [37].

To begin, we will formalize some of the results quoted in Section 2 for the FF region. The cross section for a process $X \rightarrow a c b$ is

$$
\sigma(X \rightarrow a c b)=\frac{1}{\Phi_{X}} \frac{s}{128 \pi^{3}} \int d z_{a} d z_{b}|\mathcal{M}(X \rightarrow a c b)|^{2}
$$

where $\Phi_{X}$ is the flux factor. Polarization and color indices have been suppressed. The left-hand side has been integrated over the orientation of the final state system but is otherwise exact. To write an expression involving the antenna splitting function, we approximate

$$
\mathcal{M}(X \rightarrow a c b) \approx \mathcal{M}(X \rightarrow A B) \cdot g T \cdot \frac{\mathcal{M}(\mathcal{O} \rightarrow a c b)}{\mathcal{M}(\mathcal{O} \rightarrow A B)}
$$


where $\mathcal{O}$ is the operator used in Sections $3-5$ to represent the state $A B$. The factor $g T$ is the QCD coupling and color matrix; after squaring and summing over colors, this becomes $4 \pi \alpha_{s} N_{c}$. The splitting function is defined by (2.13),

$$
\mathcal{S}\left(z_{a}, z_{c}, z_{b}\right)=s_{A B}\left|\frac{\mathcal{M}(\mathcal{O} \rightarrow a c b)}{\mathcal{M}(\mathcal{O} \rightarrow A B)}\right|^{2}
$$

Then

$$
\sigma(X \rightarrow a c b) \approx \sigma(X \rightarrow A B) \cdot \frac{\alpha_{s} N_{c}}{4 \pi} \int d z_{a} d z_{b} \mathcal{S}\left(z_{a}, z_{c}, z_{b}\right) .
$$

It is important to note that, in this formula or in (2.43), the vectors $k_{A}$ and $k_{B}$ are introduced as part of the approximation. They can be defined in any way that is consistent with the requirements that $k_{A}$ and $k_{B}$ are lightlike, $k_{A}+k_{B}=Q$, and $k_{A}$ and $k_{B}$ become parallel to $k_{a}$ and $k_{b}$, respectively, in the soft and collinear limits.

The logic of this derivation extends straightforwardly to the IF and II regions. The major change is that, in these cases, we need to introduce initial hadrons from which the initial partons are extracted.

Consider first the IF case. The cross section for a proton of momentum $P$ to scatter from a color-singlet system $X$ transferring momentum $Q$ to create a 2-parton system $c b$ is

$$
\sigma(p X \rightarrow c b)=\int d x_{a} f\left(x_{a}\right) \frac{1}{\Phi_{a X}} \frac{1}{16 \pi} \int d \cos \theta_{*}|\mathcal{M}(a X \rightarrow c b)|^{2},
$$

where $\cos \theta_{*}$ is the scattering angle in the $c b$ center of mass system. We will approximate this formula using the expression analogous to 2.43

$$
\mathcal{M}(a X \rightarrow c b) \approx \mathcal{M}(A X \rightarrow B) \cdot g T \cdot \frac{\mathcal{M}(a \mathcal{O} \rightarrow c b)}{\mathcal{M}(A \mathcal{O} \rightarrow B)} .
$$

Then the splitting function is defined by the same expression $\mathcal{S}$ as in 2.44, but now analytically continued into the new kinematic region. If a fermion line is crossed from the final to the initial state, an extra factor (-1) should be included. In addition, $s_{A B}$ in (2.44) is negative in this region, giving an extra minus sign.

The decomposition of the amplitude is illustrated in Fig. 2.5(a). The kinematics 

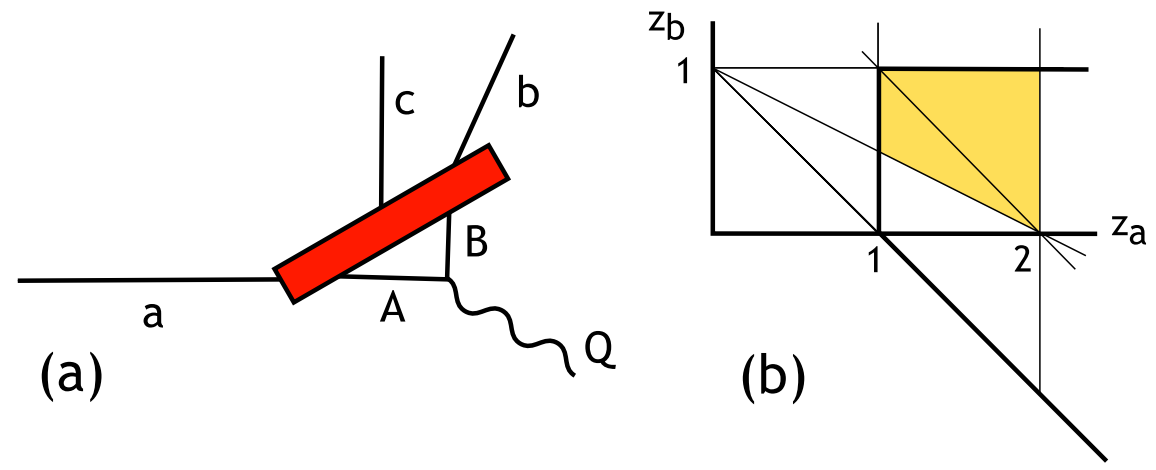

Figure 2.5: (a) Kinematics of $2 \rightarrow 3$ splitting in the initial-final (IF) case. (b) Phase space for $2 \rightarrow 3$ splitting in the IF case. The eight regions corresponding to different orderings of $\left|s_{a b}\right|,\left|s_{a c}\right|, s_{b c},\left|Q^{2}\right|$ are shown. The region that should be well described by an antenna splitting $A B \rightarrow a c b$ is shaded.

can be described by variables $y_{i j}$ and $z_{i}$ obeying the relations (2.1) to (2.4). However, the vectors $k_{A}, k_{a}$ now have negative timelike component, and the vector $Q=k_{A}+$ $k_{B}=k_{a}+k_{b}+k_{c}$ is spacelike, $Q^{2}=s_{A B}<0$. The phase space for this region covers the quadrilateral shown in Fig. 2.5(b). The region of integration is infinite, since $z_{a}$ can become very large, but the integral is cut off at large $z_{a}$ by the parton distribution function. The line $z_{a}>1, z_{b}=1$ corresponds to the region of initial state radiation, $c$ parallel to $a$. The line $z_{a}=1,0<z_{b}<1$ corresponds to the region of final state radiation, $c$ parallel to $b$. The line $z_{a}+z_{b}=1$ corresponds to $b$ parallel to $a$, that is, $b$ as initial state radiation from the primary $a$. An antenna shower should give an accurate description of the dynamics in the two regions $\left|y_{a c}\right|<\left|y_{b c}\right|<1$, $\left|y_{b c}\right|<\left|y_{a c}\right|<\left|y_{a b}\right|$ that are shaded in the figure. The new constraint $\left|y_{b c}\right|<1$ is just $\left|s_{b c}\right|<\left|Q^{2}\right|$, which is stronger than the constraint that this invariant is less than $\left|s_{a b}\right|$.

To decompose 2.46) into an appropriate form, we choose $p_{A}$ and $p_{B}$ and then change variables. Let $p_{A}$ be chosen in the direction of $p_{a}$, so that $p_{a}=z_{a} p_{A}, z_{a}>1$. Then $p_{B}=Q-p_{A}$. We have

$$
p_{a}=x_{a} P, \quad p_{A}=x_{A} P, \quad \text { so } \quad x_{a}=z_{a} x_{A}
$$


with $x_{A}$ having the definite value $x_{A}=-Q^{2} / 2 P \cdot Q$ associated with scattering a massless particle from a local current. For the reaction $a Q \rightarrow b c, s+t+u=Q^{2}$, so $t+u=Q^{2}-s=Q^{2} z_{a}$. Then

$$
t=Q^{2}\left(1-z_{b}\right)=\frac{1}{2} Q^{2} z_{a}\left(1-\cos \theta_{*}\right)
$$

Using these formulae, we can change variables from $\left(x_{a}, \cos \theta_{*}\right)$ to $\left(z_{a}, z_{b}\right)$. The Jacobian of this transformation is

$$
J=\frac{\partial\left(x_{a}, \cos \theta_{*}\right)}{\partial\left(z_{a}, z_{b}\right)}=\frac{2 x_{A}}{z_{a}}
$$

Thus,

$$
\begin{array}{r}
\sigma(p X \rightarrow c b)=\int \frac{d z_{a}}{z_{a}^{2}} \int d z_{b} \int d x_{A} x_{A} f\left(z_{a} x_{A}\right) \delta\left(x_{A}+Q^{2} / 2 P \cdot Q\right) \\
\cdot \frac{1}{\Phi_{A X}} \frac{1}{8 \pi}|\mathcal{M}(a X \rightarrow c b)|^{2}
\end{array}
$$

This is an exact rewriting of (2.46). Now apply the approximation (2.47) and group terms to form

$$
\sigma(A X \rightarrow B)=\frac{1}{\Phi_{A X}} 2 \pi \delta\left(Q^{2}+x_{A} 2 P \cdot Q\right)|\mathcal{M}(A X \rightarrow B)|^{2}
$$

Then

$$
\sigma(p X \rightarrow c b) \approx \int \frac{d z_{a}}{z_{a}^{2}} \int d z_{b} \int d x_{A} f\left(z_{a} x_{A}\right) \sigma(A X \rightarrow B) \cdot \frac{\alpha_{s} N_{c}}{4 \pi} \mathcal{S}\left(z_{a}, z_{c}, z_{b}\right)
$$

As an example, consider using this formula to describe initial-state gluon radiation in deep inelastic scattering from a quark. The total gluon emission is given by the sum of the two spin-dependent splitting functions in the fifth line of Table 2.1, equal to

$$
\sum \mathcal{S}=-\frac{z_{a}^{2}+z_{b}^{2}}{y_{a c} y_{c b}}
$$

The extra minus sign comes from the sign of $s_{A B}$ in (2.44). In the region of initial 
state radiation, $z_{a}=1 / w, z_{b} \approx 1, y_{a c}=-\left(1-z_{b}\right), y_{b c}=(1-1 / w)$. Then, setting

$$
\int d z_{b} \frac{1}{1-z_{b}}=\log \frac{Q^{2}}{\mu^{2}}
$$

we obtain

$$
\sigma(p X \rightarrow c b) \approx \int d x_{A} \int \frac{d w}{w} f\left(\frac{x_{A}}{w}\right) \sigma(A X \rightarrow B) \cdot \frac{\alpha_{s} N_{c}}{4 \pi} \frac{1+w^{2}}{(1-w)} \log \frac{Q^{2}}{\mu^{2}}
$$

which is correct.

This is an appropriate point to discuss again the signs of the expressions in Table 2.1. The antenna splitting functions are probabilities; thus, they should be positive. However, we define the splitting functions in the IF and II regions as analytic continuations of the values in the FF region, so their positivity must be checked explicitly.

As we move from the FF region to the IF region with $A$ and $a$ in the initial state, $y_{b c}$ becomes negative while all other $y_{a b}, y_{a c}, z_{a}, z_{b}$ remain positive. The factor $z_{c}$ can be negative, but $z_{c}$ does not appear in the Table. With the minus sign from $s_{A B}$ in 2.44, the denominator of $\mathcal{S}\left(z_{a}, z_{b}, z_{c}\right)$ is positive, and so we need only check the numerator functions in given in the Table. The numerator functions for $g g \rightarrow g g g$, $q \bar{q} \rightarrow q g \bar{q}$, and $q g \rightarrow q q \bar{q}$ remain positive, while the numerator functions for $g g \rightarrow \bar{q} q g$ become negative. In this last case, a fermion not present in the 2-parton system is crossed from the final to the initial state, so we must supply an extra factor $(-1)$. Then all of the expressions are positive, as required. However, if we then cross from the region $z_{b}>0$ to the region $z_{b}<0$, one $q g \rightarrow q g g$ and one $q g \rightarrow q \bar{q} q$ amplitude changes sign. This sign change is unphysical; presumably, it is due to the simple method of reconstruction in 2.26) and 2.28). We recommend setting these two amplitudes to zero for $z_{b}<0$. The region $z_{b}<0$ is outside the shaded region in Fig. 2.5(b) where we will generally use the parton shower approximation, so most likely this difficulty is not important in practice.

Similarly, for the FI region where $b$ and $B$ and taken to be in the initial state, $y_{a c}<0$. Then the numerators that go negative as we cross into the region are those 

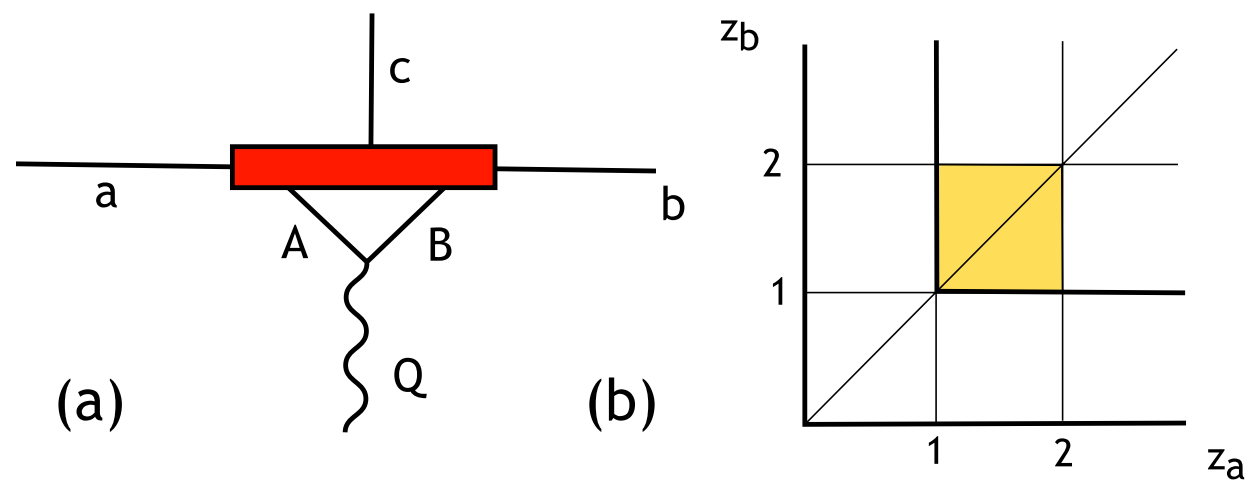

Figure 2.6: (a) Kinematics of $2 \rightarrow 3$ splitting in the initial state (II) case. (b) Phase space for $2 \rightarrow 3$ splitting in the II case. The six regions corresponding to different orderings of $\left|s_{a c}\right|,\left|s_{b c}\right|,\left|Q^{2}\right|$ are shown. The region that should be well described by an antenna splitting $A B \rightarrow a c b$ is shaded.

in the $q g \rightarrow q \bar{q} q$ cases where a fermion is crossed into the initial state. Now there are four amplitudes, one each in the $q g \rightarrow q g g$ cases and both of those in $q_{-} g_{+} \rightarrow q \bar{q} q$, that become negative when $z_{a}<0$. Again, we recommend that these amplitudes be set to zero in this region of unphysical behavior.

In the II region, both $y_{a c}$ and $y_{b c}$ are negative. The denominator of $\mathcal{S}\left(z_{a}, z_{b}, z_{c}\right)$ is positive. The numerator terms that are negative because of the sign changes are compensated by minus signs from crossing. There are no unphysical sign changes. The correct result is always obtained by taking the absolute value of the numerator expression from Table 2.1.

We now discuss the kinematics of the II case. We begin from the formula for two protons of momentum $P_{A}, P_{B}$ to produce a color-singlet system of momentum $Q$ plus a massless parton $c$,

$$
\sigma(p p \rightarrow c X)=\int d x_{a} \int d x_{b} f\left(x_{a}\right) f\left(x_{b}\right) \frac{1}{2 s_{a b}} \frac{1}{16 \pi} \int d \cos \theta_{*} \frac{2 p_{*}}{\sqrt{s_{a b}}}|\mathcal{M}(a b \rightarrow c X)|^{2}
$$

where $\cos \theta_{*}$ and $p_{*}$ are the scattering angle and the momentum in the $c X$ center of mass frame.

The decomposition of the amplitude is illustrated in Fig. 2.6(a). The kinematics 
can again be described by variables $y_{i j}$ and $z_{i}$ obeying the relations (2.1) to (2.4). Now the vectors $k_{A}, k_{a} k_{B}, k_{b}$ have negative timelike component, and the vector $Q=k_{A}+k_{B}=k_{a}+k_{b}+k_{c}$ is also negative timelike, with $Q^{2}>0$. The phase space for this region covers the quadrant shown in Fig. 2.5(b), with $z_{a}, z_{b}>1$. Again, the region of integration is infinite, but the integral is cut off by the behavior of the parton distribution functions. The line $z_{a}>1, z_{b}=1$ corresponds to the region of initial state radiation with $c$ parallel to $a$. The line $z_{a}=1, z_{b}>1$ corresponds to the region of initial state radiation with $c$ parallel to $b$. An antenna shower should give an accurate description of the dynamics in the two regions $\left|y_{a c}\right|<\left|y_{b c}\right|<1$, $\left|y_{b c}\right|<\left|y_{a c}\right|<1$ that are shaded in the figure. Again, the limit 1 here corresponds to constraints $\left|s_{a c}\right|,\left|s_{b c}\right|<\left|Q^{2}\right|$, which are stronger than the constraints that these two invariants are less than $\left|s_{a b}\right|$.

In the $a b \rightarrow c X$ process, the system $X$ must recoil with some nonzero transverse momentum. Thus, it is not possible to choose $k_{A}$ and $k_{B}$ to be parallel to $k_{a}, k_{b}$. The invariants for the $a b \rightarrow c X$ scattering process satisfy $s+t+u=Q^{2}$. Since $t=Q^{2}\left(1-z_{b}\right), u=Q^{2}\left(1-z_{a}\right)$, this means that $s=Q^{2}\left(z_{a}+z_{b}-1\right)$. Alternatively, $s=x_{a} x_{b} \cdot 2 P_{A} \cdot P_{B}$. We would like to choose the longitudinal fractions of $A$ and $B$, $x_{A}$ and $x_{B}$, to satisfy the relation

$$
x_{A} x_{B} \cdot 2 P_{A} \cdot P_{B}=Q^{2} .
$$

To make this possible, we must write

$$
x_{a}=z_{a} x_{A} \mathcal{C}, \quad x_{b}=z_{b} x_{B} \mathcal{C},
$$

with ${ }^{2}$

$$
\mathcal{C}^{2}=\frac{z_{a}+z_{b}-1}{z_{a} z_{b}}
$$

The function $\mathcal{C}\left(z_{a}, z_{b}\right)$ approaches 1 when either $z_{a}$ or $z_{b}$ goes to 1 ; that is $\mathcal{C} \approx 1$ in both collinear regions.

\footnotetext{
${ }^{2}$ Compare eq. (5.15) of 37.
} 
Also, $t+u=Q^{2}\left(2-z_{a}-z_{b}\right)=Q^{2} z_{c}$, so

$$
t=Q^{2}\left(1-z_{b}\right)=\frac{1}{2} Q^{2} z_{c}\left(1-\cos \theta_{*}\right) .
$$

We can now use (2.59) and (2.61) to change variables from $\left(x_{a}, x_{b}, \cos \theta_{*}\right)$ to $\left(x_{A}, z_{a}, z_{b}\right)$, holding $x_{B}$ fixed at the value $x_{B}=Q^{2} / x_{A} 2 P_{A} \cdot P_{B}$. The Jacobian of this transformation is

$$
J=\frac{\partial\left(x_{a}, x_{b}, \cos \theta_{*}\right)}{\partial\left(x_{A}, z_{a}, z_{b}\right)}=\frac{2 x_{B}}{z_{c}}=x_{B} \frac{Q^{2}}{s_{a b}} \frac{\sqrt{s_{a b}}}{p_{*}} .
$$

Then

$$
\begin{gathered}
\sigma(p p \rightarrow c X)=\int \frac{d z_{a}}{z_{a}^{2}} \frac{d z_{b}}{z_{b}^{2}} \frac{1}{\mathcal{C}^{4}} \int d x_{A} d x_{B} f\left(z_{a} x_{A} \mathcal{C}\right) f\left(z_{b} x_{B} \mathcal{C}\right) x_{B} \delta\left(x_{B}-Q^{2} / x_{A} 2 P_{A} \cdot P_{B}\right) \\
\cdot \frac{1}{s_{A B}} \frac{1}{8 \pi}|\mathcal{M}(a X \rightarrow c b)|^{2}
\end{gathered}
$$

This is an exact rewriting of 2.57). Now apply the approximation analogous to 2.43) or 2.47) and group terms to form

$$
\sigma(A B \rightarrow X)=\frac{1}{2 s_{A B}} 2 \pi \delta\left(Q^{2}-x_{A} x_{B} 2 P_{A} \cdot P_{B}\right)|\mathcal{M}(A X \rightarrow B)|^{2} .
$$

This gives, finally,

$\sigma(p p \rightarrow c X) \approx \int \frac{d z_{a}}{z_{a}^{2}} \frac{d z_{b}}{z_{b}^{2}} \frac{1}{\mathcal{C}^{4}} \int d x_{A} d x_{B} f\left(z_{a} x_{A} \mathcal{C}\right) f\left(z_{b} x_{B} \mathcal{C}\right) \sigma(A B \rightarrow X) \cdot \frac{\alpha_{s} N_{c}}{4 \pi} \mathcal{S}\left(z_{a}, z_{c}, z_{b}\right)$

To test this formula, consider the case of $q \bar{q}$ annihilation with the emission of a gluon collinear with the quark $a$. The sum of spin-dependent splitting functions for this case is again (2.54). In the collinear region of interest, $z_{a}=1 / w, z_{b} \approx 1$. Repeating the step that led to 2.56), we find

$$
\sigma(p p \rightarrow c X) \approx \int d x_{A} d x_{B} \int \frac{d w}{w} f\left(\frac{x_{A}}{w}\right) f\left(x_{B}\right) \sigma(A B \rightarrow X) \cdot \frac{\alpha_{s} N_{c}}{4 \pi} \frac{1+w^{2}}{(1-w)} \log \frac{Q^{2}}{\mu^{2}},
$$

which is the correct limit. 


\subsection{Comparison to previous results}

In the Introduction, we made reference to a number of previous definitions of the antenna splitting functions. We noted that these definitions agree, as they must, in the singular soft and collinear limits. However, these prescriptions differ widely away from the boundaries of phase space. In this section, we will compare our prescription to those of ARIADNE [19, 20] and Gehrmann-De Ridder, et al. [29 31].

We will make this comparison over the natural phase space discussed in the previous section-the entire $\left(z_{a}, z_{b}\right)$ plane above the line $z_{a}+z_{b}=1$. In order to describe antenna showers for initial- as well as final-state emissions, the splitting functions should extend into the region $z_{a}, z_{b}>1$. Depending on the details of how the shower is constructed, their use might be restricted to a polygon around $z_{a}=z_{b}=1$, or the expressions might be used for arbitrarily large values of $z_{a}$ and $z_{b}$.

We note again that the IF regions include the lines $z_{a}=0$ and $z_{b}=0$. Expressions for the splitting functions that are well-behaved near $z_{a}=z_{b}=1$ can possibly have a singularity on this line, though such a singularity in the middle of the phase space would be unphysical. We used this criterion in Section 4 to exclude factors of $1 / z_{a}$ and $1 / z_{b}$ from appearing in (2.28). The antenna functions of Duhr and Maltoni [28] are typically singular along this line and so cannot be used in parton shower models in all regions.

The ARIADNE and Gehrmann-De Ridder antenna functions give expressions summed over final polarizations. To compare our splitting functions to these, we must sum over a row in Table 2.1. Our summed expressions are independent of the initial polarization in the soft and collinear limits, but they depend on the polarizations of $A$ and $B$ in the interior of the $\left(z_{a}, z_{b}\right)$ space. The comparison to our expressions thus also reveals where this dependence on polarization is an important effect.

The first antenna splitting functions were put forward by the ARIADNE group [19]. Their approach started from the spin-averaged cross section for the simple splitting process $q \bar{q} \rightarrow q g \bar{q}$ in $e^{+} e^{-}$annihilation. They then guessed the expressions for the $q g \rightarrow q g g$ and $g g \rightarrow g g g$ splittings, so that these would have a similar form to 
the $q \bar{q} \rightarrow q g \bar{q}$ case,

$$
\mathcal{S}=\frac{z_{a}^{n_{a}}+z_{b}^{n_{b}}}{y_{a c} y_{b c}},
$$

where $n_{a}, n_{b}=2$ for emission from a quark and 3 for emission from a gluon.

Our philosophy, explained in Section 2, is that each individual antenna should reproduce the collinear limit predicted by QCD. These expressions are symmetric under interchange of identical particles, while 2.67) does not have this property, so we would obtain the complete splitting function by symmetrizing (2.67). This gives

$$
\begin{aligned}
& q \bar{q} \text { antenna: } \mathcal{S}=\frac{z_{a}^{2}+z_{b}^{2}}{y_{a c} y_{b c}} \\
& g g \text { antenna: } \mathcal{S}=\frac{z_{a}^{3}+z_{b}^{3}}{y_{a c} y_{b c}}+\frac{z_{a}^{3}+z_{c}^{3}}{y_{a b} y_{b c}}+\frac{z_{b}^{3}+z_{c}^{3}}{y_{a b} y_{a c}} \\
& q g \text { antenna: } \mathcal{S}=\frac{z_{a}^{2}+z_{b}^{3}}{y_{a c} y_{b c}}+\frac{z_{a}^{2}+z_{c}^{3}}{y_{a b} y_{b c}}
\end{aligned}
$$

The summed terms are each positive in the FF kinematic region. To obtain the ARIADNE splitting functions in the other regions, we analytically continue these formulae into the regions where $z_{a}$ or $z_{b}$ is greater than 1 .

The analytic continuation of the ARIADNE and, below, the Gehrmann-de Ritter results brings in the issue of the positivity of these expressions, similar to the positivity issue for our splitting functions discussed in Section 6. For the ARIADNE and Gehrmann-De Ridder antenna functions, the expressions given are summed over spins, and the individual pieces are not independent of one another. So, if they become negative, that is a problem for the complete, spin-summed, expression. For the Gehrmann-de Ridder functions, it can be seen that this happens only the regions $z_{a}<0$ and $z_{b}<0$, so this is not a serious problem. However, the ARIADNE function involve $z_{c}^{3}$, which is negative in the whole region $z_{a}+z_{b}>2$. This problem cannot be resolved by replacing $z_{c}$ with $\left|z_{c}\right|$, since this leads to expressions that do not agree with the Altarelli-Parisi factorization along the lines separating the IF regions from the II region. Fortunately, the ARIADNE functions do not become actually become negative until $z_{a}$ or $z_{b}$ becomes very large $\left(z_{a}\right.$ or $\left.z_{b} \sim 12\right)$. However, the idea that the ARIADNE functions are sums of positive and negative terms in the initial-state 
regions goes against the intuition used to propose these expressions.

We are now in a position to compare the ARIADNE function to our proposal. For the $q \bar{q}$ antenna, the expression above coincides with the sum of row 5 of Table 2.1. For the $g g$ and $g q$ cases, the ratio of the above ARIADNE functions to those defined in Table 2.1 are illustrated in Figs. 2.7, 2.8, and 2.9. The notation in the figures is the following: Each figure represents the ratio of the ARIADNE splitting function to our results for a specific initial set of polarized partons, summed over final state polarizations. The ratio goes to 1 on the lines $z_{a}=1$ and $z_{b}=1$, which correspond to the collinear limits. Away from these lines, the contours on which the ratios are $1.2,1.5,2.0,3.0$, and 5.0 (toward the + symbol), and the inverses of these numbers (toward the - symbol) are shown. The $q g$ antenna function are asymmetric between partons $a$ and $b$. The IF region in the lower right is that in which the quark is in the intial state and the gluon is in the final state. The IF region in the upper left is that in which the gluon is in the initial state and the quark remains in the final state.

The ARIADNE authors gave a different interpretation to the formulae (2.68). They took the philosophy that the collinear limit need not result from a single antenna but rather should be the result of summing over the possible antennae that would lead to a specific final state. A three gluon final state could result from any pair of the gluons radiating the third and so should be the sum of three antennae. Then the second line of (2.68) would be interpretated as the sum over these three antennae. This is a reasonable point of view for the FF kinematics considered in [19]. However, in the IF and II regions, at least one of the $z_{i}$ will be negative and so some of the terms in the last two lines of (2.68) will become negative. Such terms cannot be interpreted as independent radiators, each emittting a gluon with positive probability. It is tempting to revise the formula in (2.68) by taking the absolute values of the negative terms. However, one can readily check that no such prescription gives the correct Altarelli-Parisi limit along the lines $z_{a}=1$ and $z_{b}=1$ at the boundaries of the IF and II regions. Thus, we believe, the ARIADNE formulae can be used in the IF and II regions only by using the formulae (2.68) as written and accepting that some negative signs will appear ${ }^{3}$

\footnotetext{
${ }^{3}$ The problematical terms appear to have been simply omitted in the parton shower model of
} 

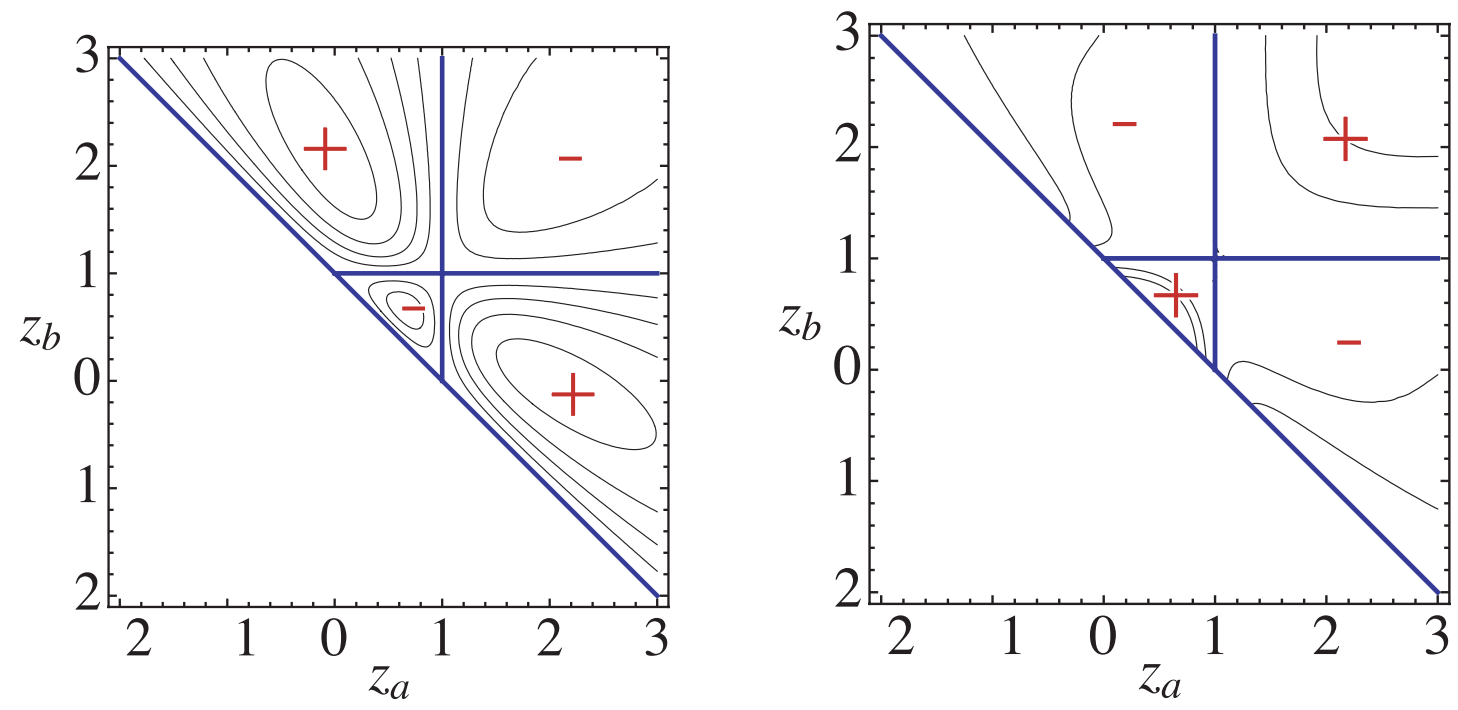

Figure 2.7: Visualization of the ratio of the ARIADNE antenna function to our antenna functions for the processes $g g \rightarrow g g g$. The figures on the left and right are the comparison of the ARIADNE antenna function to our spin-summed antenna functions from row 1 and row 2 in Table 2.1, respectively. The boundaries of phase space for the different kinematic regions are marked in blue. The contours are plotted at ratios of 1.2, 1.5, 2.0, 3.0, and 5.0, with + indicating a region in which the ratio is greater than 1. 


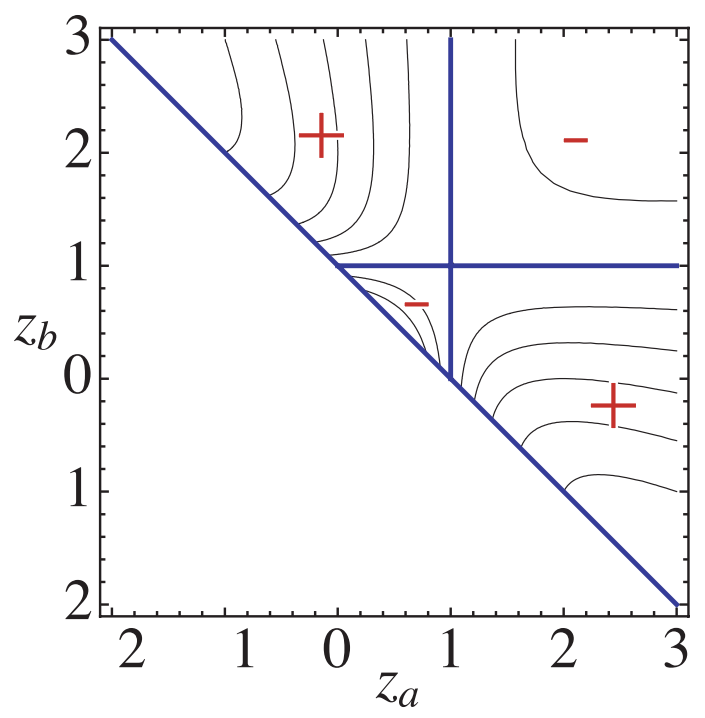

Figure 2.8: Visualization of the ratio of the ARIADNE antenna function to our antenna function for the process $q_{-} \bar{q}_{-} \rightarrow q g \bar{q}$. Our antenna function for the process $q_{-} \bar{q}_{+} \rightarrow q g \bar{q}$ coincides with the ARIADNE result and so is not included. The notation is as in Fig. 2.7.
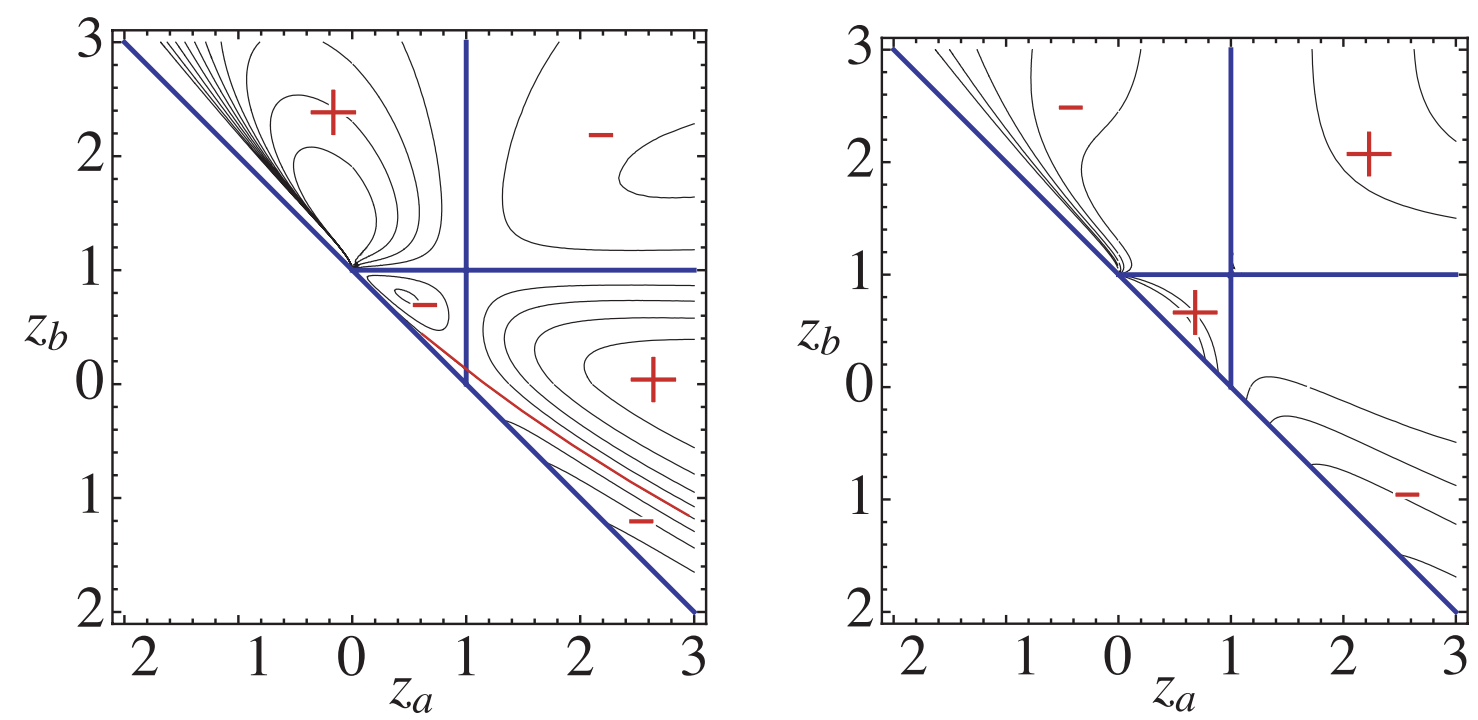

Figure 2.9: Visualization of the ratio of the ARIADNE antenna function to our antenna functions for the processes $q g \rightarrow q g g$. The figures on the left and right are the comparison of the ARIADNE antenna function to our spin-summed antenna functions from row 7 and row 8 in Table 2.1, respectively. The notation is as in Fig. 2.7. 
Gehrmann-De Ridder, Gehrmann and Glover [29 31] studied $2 \rightarrow 3$ splitting from Feynman diagrams to develop an antenna subtraction program for NNLO calculations. In doing so, they were able to extract unpolarized antenna functions for the processes $g g \rightarrow g g g, q g \rightarrow q g g$ and $q g \rightarrow q \bar{q} q$. To calculate the gluon-gluon antenna function, they used the effective Higgs coupling to gluons

$$
\mathcal{L}=-\frac{\lambda}{4} h F^{\mu \nu} F_{\mu \nu}
$$

This is essentially the same procedure that we used in Section 3, and it yields the same result as the sum of row 1 in Table 2.1. In our language, their antenna function for the gluon-gluon dipole is

$$
\mathcal{S}=\frac{y_{a c}^{2}+y_{b c}^{2}+y_{a b}^{2}+y_{a c}^{2} y_{b c}^{2}+y_{a b}^{2} y_{b c}^{2}+y_{a b}^{2} y_{a c}^{2}}{y_{a b} y_{a c} y_{b c}}+4
$$

The comparison of this antenna function to the sum of row 2 of Table 2.1 is illustrated in Fig. 2.10,

This splitting function for $g g \rightarrow g g g$ is, however, not precisely the form of the splitting function that is used in the VINCIA parton shower [21]. They use the 'global' form of the Gehrmann-De Ridder antenna function, which in our language is

$$
\mathcal{S}=\frac{1}{2}\left[\frac{2 y_{a b}^{2}+y_{a b}^{2} y_{a c}^{2}+y_{a b}^{2} y_{b c}^{2}}{y_{a b} y_{a c} y_{b c}}+\frac{8}{3}\right]
$$

To implement this antenna function, a similar procedure is used as with the ARIADNE antenna functions. That is, emissions from overlapping antenna are summed. When the three antennae contributing to $g g \rightarrow g g g$ are summed together, one recovers the result 2.70). This prescription works well in the FF kinematics. However, as in the ARIADNE case, it might require negative contributions in splitting functions for some antennae in the IF and II kinematics.

To construct the antenna functions involving quarks, Gerhmann-De Ridder, et al., 22]. We thank Jan Winter for an extensive discussion of this point.

${ }^{4}$ This expression actually differs from the one given in 29 31 by a factor of $1 / 3$, which comes from allowing any gluon to become collinear with any other gluon. Since we have identified the radiated gluon, we remove this factor. 


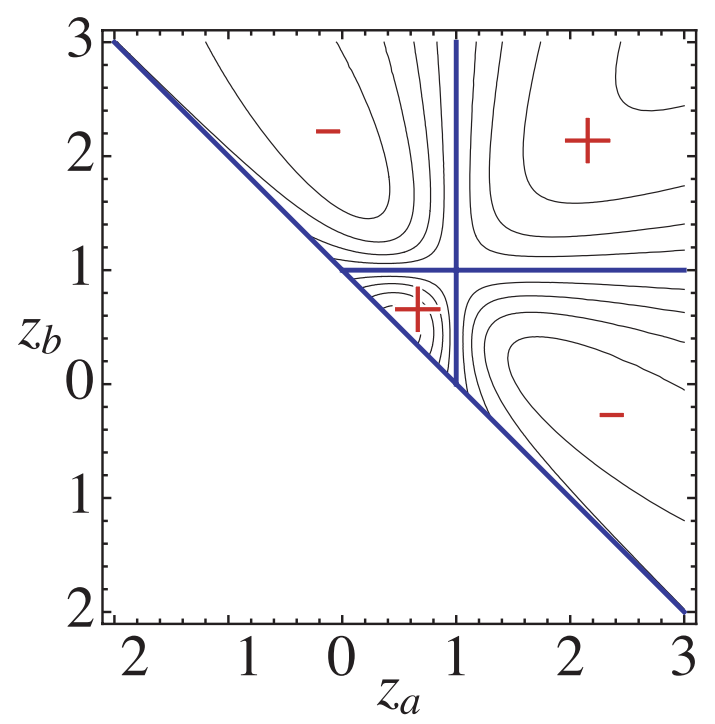

Figure 2.10: Visualization of the ratio of the Gehrmann-De Ridder antenna function to our antenna function for the process $g_{-} g_{+} \rightarrow g g g$. The antenna function for the process $g_{+} g_{+} \rightarrow g g g$ coincides with the Gehrmann-De Ridder result and so is not included. The notation is as in Fig. 2.7.

calculated the decay of a neutralino $\chi$ to a gluon and a gluino $\psi$ through the effective operator

$$
\mathcal{L}=i \eta \bar{\psi} \sigma^{\mu \nu} \chi F_{\mu \nu}+\text { h.c. }
$$

In principle, our results should agree for case of a spin $\frac{1}{2}$ initial state. However, our choices 2.26 and 2.28 for handling ambiguous momentum products, produce some differences. In our language, their antenna functions involving quarks are

$$
\begin{aligned}
& q g \rightarrow q g g: \mathcal{S}=\frac{2 y_{a b}^{2}+2 y_{a c}^{2}+y_{a b} y_{b c}^{2}+y_{a c} y_{b c}^{2}+2 y_{a c}^{2} y_{a b}^{2}}{y_{a b} y_{a c} y_{b c}}+2+2 y_{a c}+2 y_{a b} \\
& q g \rightarrow q \bar{q} q: \mathcal{S}=\frac{\left(y_{a c}+y_{a b}\right)^{2} y_{a c} y_{a b}-2 y_{a c}^{2} y_{a b}^{2}}{y_{a b} y_{a c} y_{b c}}+y_{a b}+y_{a c}
\end{aligned}
$$

The comparison to our antenna functions is illustrated in Figs. 2.11 and 2.12, For $q g \rightarrow q g g$, our result for the spin $\frac{1}{2}$ case is indeed very close to the above expression in the FF region. For $q g \rightarrow q \bar{q} q$, our prescription 2.28) gives us an extra factor of $z_{a}$ near $z_{a}=0$. 

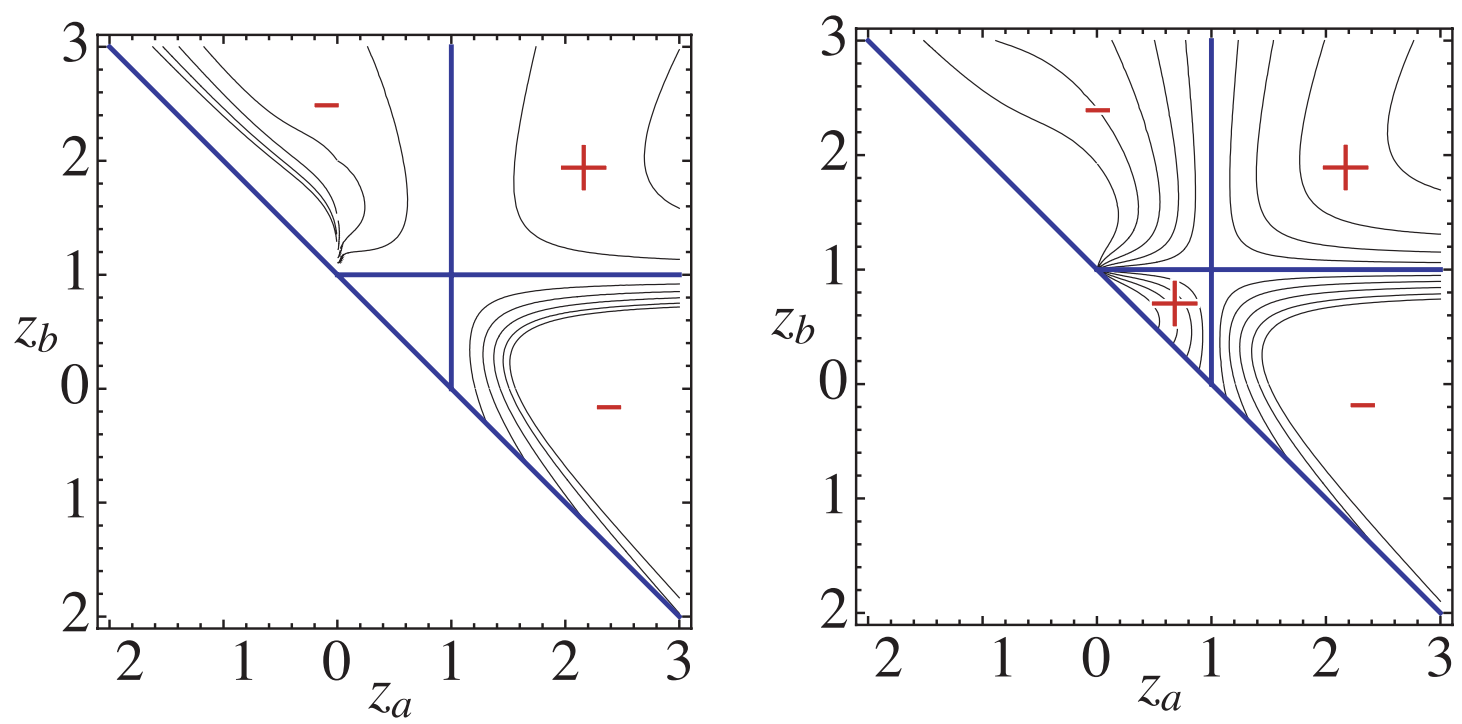

Figure 2.11: Visualization of the ratio of the Gehrmann-De Ridder antenna functions to our antenna functions for the processes $q g \rightarrow q g g$. The figures on the left and right are the comparison of the Gehrmann-De Ridder antenna function to our spin-summed antenna functions from row 7 and row 8 in Table 2.1, respectively. The notation is as in Fig. 2.7.

In summary, we have shown that the antenna splitting functions represented by (2.7) and Table 2.1 give a physically sensible prescription for the construction of antenna showers. These splitting functions can be used with the formulae (2.45), 2.53), 2.65 to generate antenna splittings in all three relevant kinematic regions. We hope that this formalism will provide a firm foundation for the construction of new parton showers based on the antenna concept. 

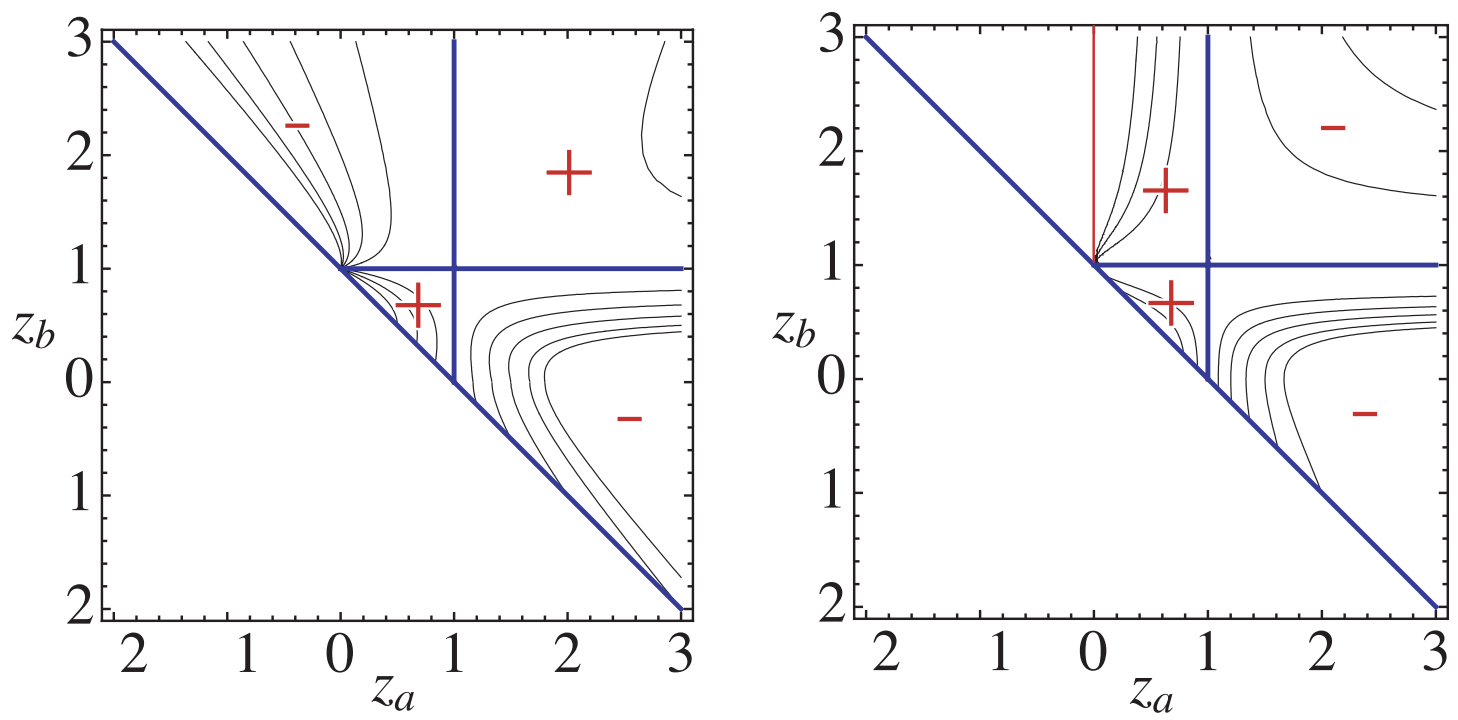

Figure 2.12: Visualization of the ratio of the Gehrmann-De Ridder antenna functions to our antenna functions for the processes $q g \rightarrow q q \bar{q}$. The figures on the left and right are the comparison of the Gehrmann-De Ridder antenna function to our spin-summed antenna functions from row 9 and row 10 in Table 2.1, respectively. The notation is as in Fig. 2.7. 


\section{Chapter 3}

\section{Antenna Splitting Functions for Massive Particles}

\subsection{Introduction}

The modeling of physics at high energy colliders relies heavily on our understanding of QCD. Quarks and gluons - collectively, partons - that are produced in high energy reactions are observed as jets of hadrons. The structure of each jet is determined by the pattern of radiation of additional partons from the original one produced in the central hard scattering reaction. For this reason, much attention has been given the past few years to the development of methods for creating parton showers, systems of partons created with the distributions predicted by QCD.

The traditional approach to the generation of parton showers is based on splitting off partons through a $1 \rightarrow 2$ branching process. This philosophy is incorporated in the widely used event generator programs PYTHIA [14] and HERWIG [15]. The construct of building a shower from $1 \rightarrow 2$ branching, often called a 'dipole shower', omits an important aspect of the physics. The longitudinal momentum distribution in the $1 \rightarrow 2$ splitting is given by the Altarelli-Parisi splitting functions [16]. In QCD, partons are emitted coherently from the two legs of a color dipole. The emission amplitude is then enhanced inside the dipole and, more importantly, cancels outside the dipole. In the 1980's, Marchesini and Webber argued that this effect could be 
incorporated into dipole showers by imposing angular ordering of emissions [18]. Thus, HERWIG is built around an angular-ordered parton shower, and PYTHIA, though it uses a different ordering scheme to choose its branchings, vetos emissions that are out of angular ordering.

Alternatively, one might build up a parton shower directly from the color dipoles, using the $2 \rightarrow 3$ process of emission of a parton by a dipole as the basic branching process. This construct is called an 'antenna shower'. The scheme was realized in the program ARIADNE, by Andersson, Gustafson, Lönnblad, and Pettersson [20] and, more recently, by the program VINCIA, by Giele, Kosower, and Skands [21]. The approach is of interest both in creating new parton shower codes for the purpose of matrix element-parton shower matching and because of its promise to yield a more accurate treatment of color dynamics in parton showers.

Recently, there has been much interest in the tagging of boosted heavy particles such as the top and Higgs observed as exotic jets [10]. Since tagging methods rely heavily on color flow, it is interesting to have a variety of approaches to the simulation of color flow in parton showers in order to test the robustness of these algorithms.

We have been engaged in providing a well-defined foundation for antenna showers, giving explicit calculations of the splitting functions that generate these showers and generalizing previous work to spin-dependent formulae. In a previous paper, we presented the complete set of spin-dependent antenna splitting functions needed to describe quark and gluon parton showers [39]. In this paper, we continue our study of this approach by presenting the spin-dependent antenna splitting functions for showers with massive particles. In constructing a shower for massless particles, spin-dependence is a convenience, especially for matching with full QCD amplitudes. For massive particles, it is more important to preserve spin information, because the decays of heavy particles such as the top quark are spin-dependent and so the experimental acceptance for the heavy particles varies significantly with their longitudinal polarization.

The formalism presented here has the same strengths and weaknesses as our previous work. We will calculate in the kinematics of final-state showers, using effective 
operators of definite spin to represent the 2-particle color dipole state before the splitting. We will work in the limit of a large number of colors in QCD for which the concept of a color dipole is strictly defined. Within this approximation, we will derive formulae for splitting functions with any ratio $m / Q$ between the mass of the particle and the mass of the two-particle system. These formulae will necessarily be less simple than those found in [39] for the massless case. We will see, though, that we can make use of spinor product formalism [33, 40] to write these splitting functions relatively compactly. The simplicity of these expressions is connected to their relation to the Maximally Helicity Violating amplitudes of QCD. This point was originally made for the massless case in [28] and is discussed in some detail in [39].

The formalism of QCD antennae was originally developed as a tool for the subtraction of infrared divergences in higher-order QCD calculations. This approach to QCD calculation was pioneered by by Kosower [25, 41]. Gehrmann-De Ridder, Gehrmann, Glover, and their students have developed this approach into a sophisticated method applicable to NLO and even NNLO computations [31, 37]. Using this formalism, Gehrmann-De Ridder, Gehrmann, and Glover have proposed forms for the spin-summed antenna splitting functions of massless quarks and gluons [29, 30]. Our previous paper reviews this latter work and compares the results from our method to theirs. There is no universal form for antenna splitting functions. The behavior of the splitting functions is prescribed in the soft and collinear limits but, away from those limits, different expressions are possible, depending on the framework used in the derivation. The systematic differences between the different proposals are explored in 39 .

Following the methods of [29, 30], splitting functions for massive, spin summed antennae were constructed in [42 44]. Again, our expressions agree with these in having the correct soft and quasi-collinear behavior but differ away from these limits. The addition of mass greatly complicates both the expressions for the splitting functions and the precise specification of the boundaries of phase space. Because of this, we do not present a detailed comparison to other massive splitting functions here.

The outline of this paper is as follows: In Section 2, we will analyze the case of gluon radiation from an antenna composed of a massive spin- $\frac{1}{2}$ fermion $(Q)$ and 
a massless spin $\frac{1}{2}$ fermion $(q)$ in a configuration of zero helicity. All of the new complications that arise when we deal with massive particles can be illustrated in this context. We will write expressions for the splitting functions in terms of spinor products of lightlike vectors associated with the massive vectors of the particles before and after the splitting. In Section 3, we will discuss the kinematics of these massive splittings and the evaluation of the the spinor product expressions.

With this introduction, we can go systematically through the various cases of antennae composed of massive and massless particles. In Sections 4 and 5 we will analyze in turn the cases of antennae with spin 0 and spin $\frac{1}{2}$ massive particles recoiling against quarks and gluons in which the antennae emits another quark or gluon. In Section 6 , we discuss the analysis of the general case of a pair of massive particles, spin 0 or spin $\frac{1}{2}$, radiating gluons. In Section 7 , we discuss antennae that create a pair of massive particles. Section 8 gives some conclusions. We collect the complete set of massive antenna splitting functions derived in this paper in Appendix A.

\subsection{The spin zero fermion-quark antenna}

The simplest case of a splitting function with massive particles arises in the system of a massive and a massless fermion created by a spin 0 operator. In this section, we will work out the spin-dependent splitting functions for this case following the prescriptions in [39]. We will then discuss the interpretation of these formulae and their comparison to the standard Altarelli-Parisi splitting functions for a massive quark [45.

In [39], each case of a spin-dependent splitting is associated with a gauge-invariant operator that creates the antenna. For this case, the required operator is

$$
\mathcal{O}=\bar{Q}_{L} q_{R}
$$

where $q$ is an ordinary quark whose mass can be ignored and $Q$ is a massive quark. 
This operator creates a 2-particle state

$$
Q_{L} \bar{q}_{L}
$$

with total spin zero about the production axis. Antennae with overall opposite helicity or with antiquarks have the same splitting functions, by the $P$ and $C$ invariance of QCD.

In [39], we wrote the basic formula for final-state antennae splitting of massless particles in the following way: Notate the splitting as $A B \rightarrow a c b$, with

$$
(A+B)^{2}=s_{A B}=Q^{2} .
$$

Throughout this paper, for any 4 -vectors $i, j$, we will define

$$
s_{i j}=(i+j)^{2}=m_{i}^{2}+2 i \cdot j+m_{j}^{2} .
$$

Let $z_{a}, z_{b}, z_{c}$ be the momentum fractions of $a, b$, and $c$ relative to their maximum value,

$$
z_{a}=\frac{2 Q \cdot q}{Q^{2}}, \text { etc. } \quad z_{a}+z_{b}+z_{c}=2 .
$$

Then the probability of a splitting is given by

$$
\int d \operatorname{Prob}=N_{c} \frac{\alpha_{s}}{4 \pi}\left(\frac{Q}{2 K}\right) \int d z_{a} d z_{b} \mathcal{S}\left(z_{a}, z_{b}, z_{c}\right) .
$$

where $N_{c}=3$ is the number of colors in QCD and $K$ is the momentum of the partons in the center of mass system of the original 2-particle antenna. In the massless case, $Q / 2 K=1$. The distribution $\mathcal{S}$ is the splitting function. In [39], we computed this function as the ratio of 3- to 2- body amplitudes of an appropriate local operator,

$$
\mathcal{S}=Q^{2}\left|\frac{\mathcal{M}(\mathcal{O} \rightarrow a c b)}{\mathcal{M}(\mathcal{O} \rightarrow A B)}\right|^{2} .
$$

This formula is still correct for the massive particle antennae discussed in this paper. We will discuss the kinematics of these antennae in more detail in Section 3. 
In the limit in which $c$ becomes collinear with $a$ or $b$, the antenna splitting functions reduce to the Altarelli-Parisi functions $P(z)$ that describe $1 \rightarrow 2$ splittings. For this limit, the formulae are not as simple in the massive case as they are in the all-massless case. We will present the explicit formulae and check them for the spin zero antenna later in this section.

To compute the amplitudes in (3.7), we use the spinor product formalism for massive particles of Schwinn and Weinzierl [46]. For a massless particle, the states of definite helicity are well-defined and Lorentz invariant. For a massive particle, the spin states depend on the frame chosen to evaluate them. In the Schwinn-Weinzierl formalism, a massless reference vector $q$ is used to define that frame. The spinors for an outgoing massive fermion of mass $m$ are written

$$
\bar{u}_{L}(p)=\frac{[q(p+m)}{\left[q p^{b}\right]} \quad \bar{u}_{R}(p)=\frac{\langle q(p+m)}{\left\langle q p^{b}\right\rangle}
$$

where the flatted vector $p^{b}$ is defined by

$$
p^{b}=p-\frac{m^{2}}{2 q \cdot p} q
$$

A particularly useful choice for $q$ is the lightlike vector in the opposite direction from $p$. Rotating coordinates so that

$$
p=(E, 0,0, p) \quad \text { with } E^{2}=p^{2}+m^{2},
$$

let

$$
p^{\sharp}=\frac{1}{2}(E+p)(1,0,0,-1),
$$

Then if we set $q=p^{\sharp}$, the flatted vector is

$$
p^{b}=\frac{1}{2}(E+p)(1,0,0,1) .
$$

This is very convenient. With this choice of $q$, the spinors defined in (3.8) are just the usual spinors of definite helicity. Using the basis of Dirac matrices where $\gamma^{5}$ is 
diagonal, it is easy to see that (3.8) reduces to

$$
\begin{aligned}
& \bar{u}_{L}=\left(\begin{array}{ll}
\sqrt{\frac{E-p}{2}} & \sqrt{\frac{E+p}{2}}
\end{array}\right) \otimes\left(\begin{array}{ll}
0 & 1
\end{array}\right) \\
& \bar{u}_{R}=\left(\begin{array}{ll}
\sqrt{\frac{E+p}{2}} & \sqrt{\frac{E-p}{2}}
\end{array}\right) \otimes\left(\begin{array}{ll}
1 & 0
\end{array}\right) .
\end{aligned}
$$

Using these conventions, we can easily compute the 2 particle matrix elements of the operator (3.1). Denote the momenta of the initial-state heavy quark and light antiquark as $A$ and $B$, respectively. Then

$$
\begin{aligned}
& \mathcal{M}\left(Q_{L} \bar{q}_{L}\right)=\frac{[q A B\rangle}{\left[q A^{b}\right]}=\left\langle A^{b} B\right\rangle \\
& \mathcal{M}\left(Q_{R} \bar{q}_{L}\right)=\frac{m\langle q B\rangle}{\left\langle q A^{b}\right\rangle}
\end{aligned}
$$

The helicity of the $\bar{q}$ must be $L$, but the heavy quark created by (3.1) could be in either spin state. However, with the usual definition of helicity, the production of $Q_{R} \bar{q}_{L}$ from a spin 0 operator would be forbidden by angular momentum. Indeed, when we set $q=A^{\sharp}$,

$$
\mathcal{M}\left(Q_{R} \bar{q}_{L}\right) \sim\left\langle A^{\sharp} B\right\rangle=0,
$$

because $A^{\sharp}$ is a lightlike vector parallel to $B$. The only nonzero matrix element is then

$$
\mathcal{M}\left(Q_{L} \bar{q}_{L}\right)=\left\langle A^{b} B\right\rangle ;
$$

this gives the denominator in (3.6). It is convenient that

$$
\left|\left\langle A^{b} B\right\rangle\right|^{2}=Q^{2}-m^{2}=2 Q K,
$$

with $K$ as in (3.6).

It is straightforward to work out the numerator of (3.6) for the four possible spin states of the 3-particle system $Q g q_{L}$. As in [39], we label the three final-state momenta as $(a, c, b)$, with the emitted particle as $c$. The results, using a general reference vector 
$q$ in $(3.8)$, are

$$
\begin{aligned}
& \mathcal{M}\left(Q_{L} g_{L} \bar{q}_{L}\right)=-\frac{1}{[q c]}\left\{\frac{\left\langle c a^{b}\right\rangle[q Q b\rangle}{s_{a c}-m^{2}}+\frac{\left[q Q a^{b}\right\rangle}{[b c]}\right\} \\
& \mathcal{M}\left(Q_{L} g_{R} \bar{q}_{L}\right)=-\frac{\left\langle a^{b} b\right\rangle[c Q b\rangle}{\langle b c\rangle\left(s_{a c}-m^{2}\right)} \\
& \mathcal{M}\left(Q_{R} g_{L} \bar{q}_{L}\right)=-\frac{m}{\left[a^{b} c\right]\left\langle q a^{b}\right\rangle}\left\{\frac{\langle c q\rangle\left[a^{b} Q b\right\rangle}{s_{a c}-m^{2}}+\frac{\left[a^{b} Q q\right\rangle}{[b c]}\right\} \\
& \mathcal{M}\left(Q_{R} g_{R} \bar{q}_{L}\right)=-\frac{m\langle q b\rangle[c Q b\rangle}{\langle b c\rangle\left\langle q a^{b}\right\rangle\left(s_{a c}-m^{2}\right)}
\end{aligned}
$$

We have omitted the overall factor of $\left(g T^{a}\right)$. When we put $q=a^{\sharp}$, we can recognize the simplification

$$
\left[a^{\sharp} Q a^{b}\right\rangle=\left[a^{b} Q a^{\sharp}\right\rangle=0 .
$$

This follows from the fact that the 4-vector $Q$ is a linear combination of the two lightlike vectors $a^{b}$ and $a^{\sharp}$. Now square these expressions and combine with (3.17) to evaluate (3.7). This gives

$$
\begin{aligned}
\mathcal{S}\left(Q_{L} g_{L} \bar{q}_{L}\right) & =\frac{Q}{2 K}\left|\frac{\left\langle a^{b} c\right\rangle\left[a^{\sharp} Q b\right\rangle}{\left[a^{\sharp} c\right][c a c\rangle}\right|^{2} \\
\mathcal{S}\left(Q_{L} g_{R} \bar{q}_{L}\right) & =\frac{Q}{2 K}\left|\frac{\left\langle a^{b} b\right\rangle[c Q b\rangle}{\langle b c\rangle[c a c\rangle}\right|^{2} \\
\mathcal{S}\left(Q_{R} g_{L} \bar{q}_{L}\right) & =\frac{m^{2} Q}{2 K}\left|\frac{\left\langle a^{\sharp} c\right\rangle\left[a^{b} Q b\right\rangle}{\left\langle a^{\sharp} a^{b}\right\rangle\left[a^{b} c\right][c a c\rangle}\right|^{2} \\
\mathcal{S}\left(Q_{R} g_{R} \bar{q}_{L}\right) & =\frac{m^{2} Q}{2 K}\left|\frac{\left\langle a^{\sharp} b\right\rangle[c Q b\rangle}{\left\langle a^{\sharp} a^{b}\right\rangle\langle b c\rangle[c a c\rangle}\right|^{2}
\end{aligned}
$$

In the all-massless case, we managed to produce antenna splitting functions that were simple rational functions of the $z_{a}$ [39]. Here, the antenna splitting functions are more complicated, but not excessively so. The main complications come from the denominators $\left(s_{a c}-m^{2}\right)=[c a c\rangle$, which do not factorize simply, and from the multiple lightlike vectors needed to characterize the state of the massive quark. In this case, 
it is not so difficult to write the splitting functions in terms of 4-vector products:

$$
\begin{aligned}
\mathcal{S}\left(Q_{L} g_{L} \bar{q}_{L}\right) & =\frac{Q}{K} \frac{s_{a^{b} c}\left(2 a^{\sharp} \cdot Q b \cdot Q-a^{\sharp} \cdot b Q^{2}\right)}{s_{a^{\sharp} c}\left(s_{a c}-m^{2}\right)^{2}} \\
\mathcal{S}\left(Q_{L} g_{R} \bar{q}_{L}\right) & =\frac{Q}{K} \frac{s_{a^{\natural} b}\left(2 b \cdot Q c \cdot Q-b \cdot c Q^{2}\right)}{s_{b c}\left(s_{a c}-m^{2}\right)^{2}} \\
\mathcal{S}\left(Q_{R} g_{L} \bar{q}_{L}\right) & =\frac{m^{2} Q}{K} \frac{s_{a^{\sharp} c}\left(2 a^{b} \cdot Q b \cdot Q-a^{b} \cdot b Q^{2}\right)}{s_{a^{\sharp} a^{b}} s_{a^{b} c}\left(s_{a c}-m^{2}\right)^{2}} \\
\mathcal{S}\left(Q_{R} g_{R} \bar{q}_{L}\right) & =\frac{m^{2} Q}{K} \frac{s_{a^{\sharp} b}\left(2 b \cdot Q c \cdot Q-b \cdot c Q^{2}\right)}{s_{a^{\sharp} a^{b}} s_{b c}\left(s_{a c}-m^{2}\right)^{2}}
\end{aligned}
$$

However, the structure of the expressions is more clearly visible in the form 3.20 .

The expressions (3.20) contain exact tree-level matrix elements for the transition of the operator $\mathcal{O}$ to a three-particle state. They are correctly used in a parton shower for any values of $m / Q$ and $p_{T} / Q$ among the final-state particles, as long as the virtuality at the previous and successive branchings of the shower are well separated from $Q$. In the all-massless case discussed in [39], we made approximations to the splitting functions valid in the soft and collinear limits. It is less obvious here which approximations are appropriate, and, in any case, we did not see how to achieve much further simplification. So we will stop at this point for this set of splitting fuctions and for all of the massive particle splitting functions quoted in this paper.

To evaluate expressions of the type of (3.20), we find it easiest not to convert the expressions in (3.20) into 4-vector products or dimensionless scalars built from these but, rather, to directly evaluate the spinor brackets. We will discuss a strategy to evaluate these brackets in the next section.

Finally, we must discuss the collinear limits and the connection to the the AltarelliParisi splitting functions. For the spin zero antennae, this connection is easiest to discuss for the limit $c \| b$, where only massless particles are involved. We must still take account of the fact that, because $b$ and $c$ recoil against a massive particle, their maximum momentum is limited. To account for this, let

$$
\tilde{z}_{b, c}=\frac{z_{b, c}}{\left(1-m^{2} / Q^{2}\right)} .
$$


so that $\tilde{z}_{b}$ and $\tilde{z}_{c}$ run from 0 to 1 and, in the limit $c \| b, \tilde{z}_{b}+\tilde{z}_{c}=1$. Then, in this collider limit, $\mathcal{S}$ has the singularity

$$
\mathcal{S} \sim \delta_{a, A} \frac{Q^{2}}{s_{b c}} P_{B \rightarrow c}\left(\tilde{z}_{c}\right)
$$

The expressions in (3.20) satisfy this relation. The splitting functions to $Q_{R} g_{L, R}$ must have no collinear singularity. This follows from the fact that $\left[a^{b} Q b\right\rangle$ and $\left\langle a^{\sharp} b\right\rangle$ vanish when $b$ becomes opposite to $a$. The cases of $Q_{L} g_{L, R}$ do have singularities proportional to $s_{a^{\sharp} c}^{-1}$ and $s_{b c}^{-1}$, with the correct coefficients to match 3.22.

In the limit $c \| a$, where the $1 \rightarrow 2$ splitting involves a massive particle, the limit is slightly more complicated. For the splitting of a massive particle, the usual Altarelli-Parisi formula for the collinear splitting is conventionally rewritten as

$$
\int d \operatorname{Prob}=N_{c} \frac{\alpha_{s}}{2 \pi} \int d z \int \frac{d p_{T}^{2}}{\left(p_{T}^{2}+z^{2} m^{2}\right)} P\left(z, p_{T}\right) .
$$

We divide the usual expressions for $P\left(z, p_{T}\right)$ by 2 so that these functions give the contribution from one of the two antennae that contribute to a collinear singularity. Mass-suppressed terms can contain an additional factor of $\left(p_{T}^{2}+z^{2} m^{2}\right)$ in the denominator; this is why we have allowed the Altarelli-Parisi function to depend on $p_{T}$. With this formalism, for $c$ becoming parallel to $a$,

$$
\mathcal{S}\left(z_{a}, z_{b}, z_{c}\right) \rightarrow \frac{Q^{2}}{s_{a c}-m_{A}^{2}} P\left(\tilde{z}_{c}, p_{T}\right)
$$

where $s_{a c}=(a+c)^{2}$. Here again, the parameter $\tilde{z}_{c}$ must be scaled to equal 1 at its maximum value, as in (3.22). For the present case in which the (ac) system recoils against a massless parton, $\tilde{z}_{c}=z_{c}$.

To discuss the limits $c \| a$, we first need to recall the Altarelli-Parisi functions for splitting of a gluon from a massive fermion. The Altarelli-Parisi functions are defined in the limit of not only collinear but also high energy emission. For a particle of energy $E$ splitting to particles with transverse momentum $p_{T}$ and finite masses $m_{i}$, these functions describe the regime $p_{T} \sim m_{i} \ll E$. For a splitting $Q \rightarrow g Q$, as we 
have in this case, the spin-summed splitting function is [45]

$$
P(z)=\frac{1+(1-z)^{2}}{z}-\frac{m^{2}}{a \cdot c}
$$

This expression becomes clearer when it is written as a set of spin-dependent AltarelliParisi functions. In the convention defined by (3.24),

$$
\begin{aligned}
P\left(Q_{L} \rightarrow Q_{L} g_{L}\right) & =\frac{p_{T}^{2}}{p_{T}^{2}+z^{2} m^{2}} \frac{1}{z} \\
P\left(Q_{L} \rightarrow Q_{L} g_{R}\right) & =\frac{p_{T}^{2}}{p_{T}^{2}+z^{2} m^{2}} \frac{(1-z)^{2}}{z} \\
P\left(Q_{L} \rightarrow Q_{R} g_{L}\right) & =\frac{m^{2}}{p_{T}^{2}+z^{2} m^{2}} \frac{z^{4}}{z} \\
P\left(Q_{L} \rightarrow Q_{R} g_{R}\right) & =0
\end{aligned}
$$

The sum of these terms does reproduce (3.26). The placement of the factors of $z$ implements the dead cone in which soft radiation from a massive particle is suppressed within a cone of size $1 / \gamma$, where $\gamma$ is the boost of the heavy particle [47, 48].

We can now compare the $c \| a$ limits of our antenna splitting functions to (3.27). In the collinear limit,

$$
s_{a c}-m^{2}=\frac{p_{T}^{2}+z^{2} m^{2}}{z(1-z)} .
$$

Using this formula and the collinear limits of the spinor products, we find that 3.20 does satisfy (3.25) with (3.27), up to corrections of relative order $m^{2} / Q^{2}$. In particular, in the limit $c \| a, a^{\sharp}$ becomes collinear with $b$. Then the vanishing of $\left\langle a^{\sharp} b\right\rangle$ with no compensatory vanishing in the denominator gives the zero in the last line of (3.27).

The spin-dependent splitting functions in the remaining sections of this paper also satisfy these checks on the collinear limits. For convenience, we list the complete set of mass-dependent, spin-dependent Altarelli-Parisi splitting functions that are needed for these checks in Appendix B. 


\subsection{Kinematics of massive antennae}

The splitting functions computed in the previous section were written in terms of spinor products of massless vectors associated with the massive 4-vectors of the antenna. One should ask, how are these massless vectors computed? A similar question arises in the context of the formula $(3.6)$ for the antenna splitting probability. This equation is easily written down as the ratio of a cross section to produce a 3body final state, integrated over 3-body phase space, to the cross section to produce a 2-body final state, without a radiated parton, integrated over 2-body phase space. In particular, the integral $\int d z_{a} d z_{b}$ is an integral over 3-body phase space. One should ask, what is the boundary of the region of integration for these variables, and how does one sample points in the interior of this region?

For massless antenna, the answers to these questions are straightforward. For antenna with both radiators in the final state (FF antennae in the notation of [39]), the complete phase space region is the triangle

$$
0<z_{a}, z_{b}<1 \quad z_{a}+z_{b}>1
$$

and the region well described by the radiation process $A B \rightarrow a c b$, with $c$ soft, is the smaller region where

$$
0<z_{c}<z_{a}<1 \text { and } 0<z_{c}<z_{b}<1
$$

To create an additional radiated particle in a state with $N$ massless particles, we choose a color-connected pair of particles $A B$, boost so that $A$ and $B$ are of equal length and back-to-back, choose $\left(z_{a}, z_{b}\right)$ as a random point in the region $(3.30)$, replace the 2-particle system $A B$ by the chosen 3-particle system $a c b$, and, finally, reverse the boost to bring $a c b$ back into the original frame. The corresponding phase space regions and algorithms for antennae including initial-state particles are described in [39]. In this paper, however, we will only discuss final-state showers.

We believe that these 4-vector configurations for massless particles provide a good starting point for constructing 4-vector configurations that include massive particles. 
Given a point $\left\{\ell_{i}\right\}$ in the phase space of of $N$ massless particles, one can obtain a point $\left\{k_{i}\right\}$ in the phase space of $N$ massive particles by rescaling

$$
\vec{k}_{i}=\lambda \vec{\ell}_{i}
$$

where $\lambda$ obeys

$$
\sum_{i} \hat{E}_{i}=E_{\mathrm{CM}}, \quad \text { with } \quad \hat{E}_{i}=\left(\left|\lambda \vec{\ell}_{i}\right|^{2}+m_{i}^{2}\right)^{1 / 2}
$$

Conversely, every point of the massive phase space can be constructed uniquely in this way. The scale factor $\lambda$ is close to unity unless one of the massive particles is nonrelativistic. The relation of the phase space measures for the massive and massless variables is [49]

$$
d \Pi_{N}(k)=d \Pi_{N}(\ell) \cdot \lambda^{2 N-4} \prod_{i} \frac{\left|\lambda \vec{\ell}_{i}\right|}{E_{i}} \frac{\sum_{i}\left|\lambda \vec{\ell}_{i}\right|}{\sum_{i}\left|\lambda \vec{\ell}_{i}\right|^{2} / E_{i}} .
$$

We will refer to the massless vectors $\left\{\ell_{i}\right\}$ as the backbone of the massive configuration.

We now have a strategy for the constructing the $N$ particle phase space of a parton shower that involves massive particles. Starting with a system of 2 massless particles, construct a shower of massless vectors according to the procedure described above. In each antenna, let the momentum fractions of the (massless) final particles $a, b$ be $w_{a}, w_{b}$. Rescale within the antenna by $\lambda$ and use the massless vectors and this value of $\lambda$ to compute the splitting probabilities. For example, for the splitting described in the previous section with particle $a$ massive, the equation for $\lambda$ is

$$
E_{a}+\lambda\left(\left|\vec{\ell}_{b}\right|+\left|\vec{\ell}_{c}\right|\right)=Q
$$

The splitting probability is given by

$$
\int d \operatorname{Prob}=N_{c} \frac{\alpha_{s}}{4 \pi}\left(\frac{Q}{2 K}\right) \int d w_{a} d w_{b} \cdot \lambda^{2} \cdot\left(\frac{\lambda w_{a}}{E_{a}}\right) \frac{\lambda}{\left|\lambda w_{a}\right|^{2} Q / 2 E_{a}+\lambda\left(w_{b}+w_{c}\right)} \mathcal{S} .
$$

To evaluate the splitting function $\mathcal{S}$ we need the flatted and sharped vectors $a^{b}$ and 
$a^{\sharp}$. The first of these is given by

$$
a^{b}=\frac{1}{2}\left(E_{a} / \lambda\left|\vec{\ell}_{a}\right|+1\right) \lambda \ell_{a}
$$

and $a^{\sharp}$ is the massless vector of the same length pointing in the opposite direction. Once the configuration is chosen, the three new massless vectors are boosted back to the frame of the shower, and we are ready to generate the next antenna. When the shower is completed, the entire backbone must be rescaled to put the final massive particles on shell. In this prescription, the recoil due to emissions is done locally in each antenna to the extent that the particles are relativistic, but the recoil for nonrelativistic massive particles is distributed over the whole shower.

There is one more complication that should be discussed. For a massless particle, the spin state is determined by the helicity in a way that is independent of frame. For a massive particle, a change of frame can rotate the spin. The helicity is preserved by rotations and by boosts along the direction of motion. Other boosts, at an angle to the direction of motion, change the spin orientation. In the massive particle shower described here, we ignore this effect. In any event, it is unimportant when the massive particles are relativistic, and this accounts for most of the radiation from these particles.

\subsection{Antennae with a massive spin 0 particle}

We are now ready to put together a catalogue of the antenna splitting functions that describe the emission of quarks and gluons in the showering of massive particles. We begin with the case of a spin 0 massive particle $S$ recoiling against a quark or a gluon.

In the quark case, the antenna is described by an operator

$$
\mathcal{O}=S^{\dagger}\langle 2| q_{R}
$$

where $\langle 2|$ is a spin- $\frac{1}{2}$ spurion that controls the quark polarization. Here and in the rest of the paper, we will analyze a subset of the various discrete choices from which 
the rest can be derived using the $P$ and $C$ symmetries of QCD. Here, for example, the two cases

$$
S \bar{q}_{L} \rightarrow S g_{L} \bar{q}_{L} \text { and } S \bar{q}_{L} \rightarrow S g_{R} \bar{q}_{L}
$$

considered below suffice to provide all of the possble spin-dependent splitting functions for $S \bar{q} \rightarrow S g \bar{q}$ and $S q \rightarrow S g q$.

The 2-particle matrix element of the operator $(3.37)$ is

$$
\mathcal{M}\left(\mathcal{O} \rightarrow S \bar{q}_{L}\right)=\langle 2 B\rangle .
$$

Then, for the 2-particle antenna $S \bar{q}_{L}$ with $S$ moving the $\hat{3}$ direction, 2 should be a massless fermion moving parallel to $S$. In the following, we will set $2=A^{b}$. This choice follows the methods used in [39]. In that paper, the polarization vectors associated with operators $\mathcal{O}$ with nonzero spin are built from massless vectors 1 and 2 , chosen in the directions of $B$ and $A$, respectively. With this choice, the denominator of the expression (3.7) for the splitting function is again evaluated as (3.17).

The 3-particle matrix elements of (3.37) are

$$
\begin{aligned}
\mathcal{M}\left(\mathcal{O} \rightarrow S g_{L} \bar{q}_{L}\right) & =\frac{\left\langle A^{b}(b+c) a c\right\rangle}{[c a c\rangle[b c]} \\
\mathcal{M}\left(\mathcal{O} \rightarrow S g_{R} \bar{q}_{L}\right) & =-\frac{\left\langle A^{b} b\right\rangle\langle b a c]}{[c a c\rangle\langle b c\rangle}
\end{aligned}
$$

Here again, we strip off the factors of $g$ and color matrices. The final results are surprisingly compact.

For an antenna containing a massive scalar and gluon, we need to find an operator that defines an antenna whose initial state includes a gluon of a definite polarization. For the antenna with a left-handed gluon, we may choose [39]

$$
\mathcal{O}=\frac{i}{\sqrt{2}} S^{\dagger}\langle 2|\bar{\sigma} \cdot F| 2\rangle
$$

where

$$
\bar{\sigma} \cdot F=\frac{1}{2} \bar{\sigma}^{m} \sigma^{n} F_{m n} .
$$


This operator projects onto anti-self-dual gauge fields or left-handed physical gluons. The corresponding operator $\sigma \cdot F$ can be used to define the antenna with an initial right-handed gluon. The two-particle matrix elements of (3.41) are

$$
\mathcal{M}\left(\mathcal{O} \rightarrow S g_{L}\right)=\langle 2 B\rangle^{2} \quad \mathcal{M}\left(\mathcal{O} \rightarrow S g_{R}\right)=0 .
$$

The zero for a $g_{R}$ is just as one should have expected. As above, we set $2=A^{b}$.

There are two types of 3-particle matrix elements of (3.41). First, the antenna can radiate a gluon. The corresponding matrix elements are

$$
\begin{aligned}
& \mathcal{M}\left(\mathcal{O} \rightarrow S g_{L} g_{L}\right)=\frac{1}{[b c]}\left[\frac{\left\langle A^{b} b\right\rangle^{2}[b a c\rangle}{[c a c\rangle}+2\left\langle A^{b} c\right\rangle\left\langle A^{b} b\right\rangle+\frac{\left\langle A^{b} c\right\rangle^{2}[c a b\rangle}{[b a b\rangle}\right] \\
& \mathcal{M}\left(\mathcal{O} \rightarrow S g_{R} g_{L}\right)=-\frac{\left\langle A^{b} b\right\rangle^{2}\langle b a c]}{[c a c\rangle\langle b c\rangle} \\
& \mathcal{M}\left(\mathcal{O} \rightarrow S g_{L} g_{R}\right)=-\frac{\left\langle A^{b} c\right\rangle^{2}\langle c a b]}{[b a b\rangle\langle b c\rangle} \\
& \mathcal{M}\left(\mathcal{O} \rightarrow S g_{R} g_{R}\right)=0,
\end{aligned}
$$

following the pattern established in 3.40). Second, the gluon may split into a quarkantiquark pair. For this, we need the matrix elements

$$
\begin{aligned}
& \mathcal{M}\left(\mathcal{O} \rightarrow S \bar{q}_{R} q_{L}\right)=-\frac{\left\langle A^{\mathrm{b} b}\right\rangle^{2}}{\langle b c\rangle} \\
& \mathcal{M}\left(\mathcal{O} \rightarrow S \bar{q}_{L} q_{R}\right)=\frac{\left\langle A^{b} c\right\rangle^{2}}{\langle b c\rangle} .
\end{aligned}
$$

The splitting functions derived from these matrix elements using (3.7) are listed systematically in Appendix A.

\subsection{Antennae with a massive spin $\frac{1}{2}$ particle}

In the same way, we can construct operators that correspond to the initial states of antennae involving a massive Dirac fermion $Q$ with a quark or gluon. The massive 
fermion can have helicity $\pm \frac{1}{2}$. Because the $Q$ is massive, an initial left-handed $Q$ can flip over after radiation to a right-handed $Q$, or vice versa. We have seen this already in the special case considered in Section 2. In this section, we will recall the results from Section 2 and compare them to those of the other three possible antennae of this type.

The antennae with an initial state containing $F$ and a quark can be arranged in a state with total spin about the axis of motion $\left|J^{3}\right|$ equal to 0 or 1 . The spin 0 case was considered in Section 2. The appropriate operator $\mathcal{O}$ is

$$
\mathcal{O}=\bar{Q} q_{R}
$$

The matrix elements of this operator between two-particle $F \bar{q}$ states are

$$
\mathcal{M}\left(\mathcal{O} \rightarrow Q_{L} \bar{q}_{L}\right)=\left\langle A^{b} B\right\rangle \quad \mathcal{M}\left(\mathcal{O} \rightarrow Q_{L} \bar{q}_{R}\right)=0
$$

in our convention that $A^{\sharp}$ should be used as the reference vector for $Q$. The threeparticle matrix elements are then readily computed. If we use $a^{\sharp}$ from the beginnning as the reference vector for $Q,(3.18)$ gives

$$
\begin{aligned}
\mathcal{M}\left(Q_{L} g_{L} \bar{q}_{L}\right) & =-\frac{\left\langle c a^{b}\right\rangle\left[a^{\sharp} Q b\right\rangle}{\left[a^{\sharp} c\right][c a c\rangle} \\
\mathcal{M}\left(Q_{L} g_{R} \bar{q}_{L}\right) & =-\frac{\left\langle a^{b} b\right\rangle[c Q b\rangle}{\langle b c\rangle[c a c\rangle} \\
\mathcal{M}\left(Q_{R} g_{L} \bar{q}_{L}\right) & =-m \frac{\left\langle c a^{\sharp}\right\rangle\left[a^{b} Q b\right\rangle}{\left[a^{b} c\right]\left\langle a^{\sharp} a^{b}\right\rangle[c a c\rangle} \\
\mathcal{M}\left(Q_{R} g_{R} \bar{q}_{L}\right) & =-m \frac{\left\langle a^{\sharp} b\right\rangle[c Q b\rangle}{\langle b c\rangle\left\langle a^{\sharp} a^{b}\right\rangle[c a c\rangle} .
\end{aligned}
$$

The antenna splitting function can be constructed from these elements in the manner described in Section 2.

The spin 1 case can be treated in the same way. As described in [39] and at the beginning of Section 4, we introduce lightlike vectors 1 and 2 in the direction of $B$ 
and $A$, respectively. Then an appropriate operator to define this antenna is

$$
\mathcal{O}=\bar{Q} 1\rangle\left[2 q_{L}\right.
$$

The two-particle matrix elements of this operator are

$$
\mathcal{M}\left(\mathcal{O} \rightarrow Q_{L} \bar{q}_{R}\right)=\left\langle A^{b} 1\right\rangle\langle 2 B\rangle, \quad \mathcal{M}\left(\mathcal{O} \rightarrow Q_{R} \bar{q}_{R}\right)=0,
$$

Thus, this operator does correctly represent the initial situation. We will set $2=A^{b}$ and $1=B$ in the following expressions.

The splitting function for the antenna to radiate a gluon is computed from the three-particle matrix elements of this operator to $F g \bar{q}$ final states. These are

$$
\begin{aligned}
\mathcal{M}\left(Q_{L} g_{R} \bar{q}_{R}\right) & =-\frac{\left\langle a^{b} B\right\rangle\left[A^{b}(b+c) a c\right]}{\langle c a c]\langle b c\rangle} \\
\mathcal{M}\left(Q_{L} g_{L} \bar{q}_{R}\right) & =-\frac{\left[A^{b} b\right]}{\langle c a c][b c]\left[a^{\sharp} a^{b}\right]}\left\{\left[a^{\sharp} a c\right\rangle[b Q B\rangle+m^{2}\langle c B\rangle\left[a^{\sharp} b\right]\right\} \\
\mathcal{M}\left(Q_{R} g_{R} \bar{q}_{R}\right) & =\frac{m\left\langle a^{\sharp} B\right\rangle\left[A^{b}(b+c) a c\right]}{\left\langle a^{\sharp} a^{b}\right\rangle\langle c a c]\langle b c\rangle} \\
\mathcal{M}\left(Q_{R} g_{L} \bar{q}_{R}\right) & =\frac{m\left[A^{b} b\right]}{\left\langle a^{\sharp} a^{b}\right\rangle\langle c a c]\langle b c\rangle}\left\{\left\langle a^{\sharp} B\right\rangle\langle c a b]+\left\langle a^{\sharp} c\right\rangle\langle B c b]\right\} .
\end{aligned}
$$

The splitting functions derived from these formulae and those in (3.48) are catalogued in Appendix A.

For the antennae with $Q$ and a gluon, we again use the operator $\bar{\sigma} \cdot F$ to define the initial state as containing a gluon of definite left-handed polarization. There are two cases, with total spin $\frac{1}{2}$ and $\frac{3}{2}$. For the spin $\frac{1}{2}$ case, the appropriate operator is

$$
\mathcal{O}=-\frac{i}{\sqrt{2}} \bar{Q} \bar{\sigma} \cdot F|2\rangle
$$

The dominant two-particle matrix element of this operator is

$$
\mathcal{M}\left(\mathcal{O} \rightarrow Q_{L} \bar{g}_{L}\right)=\left\langle A^{b} B\right\rangle\langle 2 B\rangle
$$




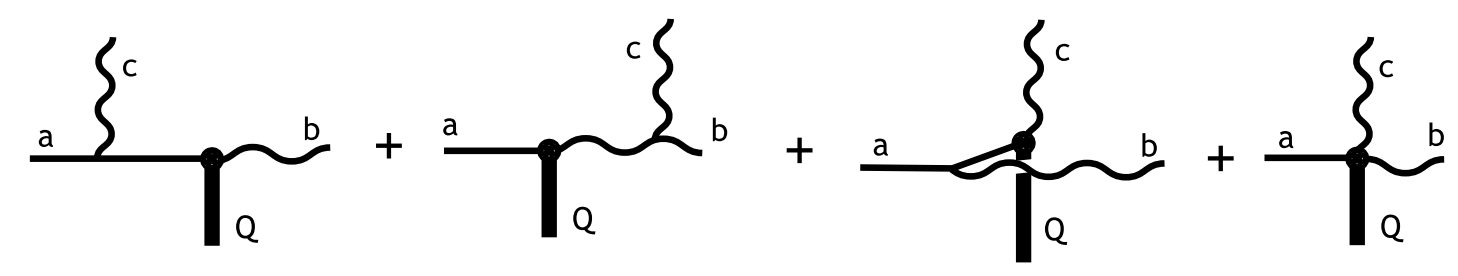

Figure 3.1: Feynman diagrams for the computation of the $F g \rightarrow F g g$ splitting functions [39].

If we recall that the vector 2 is identified with $A^{\text {b }}$, we see that this puts the initial $Q$ and $g$ into just the correct orientation. The matrix elements to $Q_{L} g_{R}, Q_{R} g_{L}$, and $Q_{R} g_{R}$ all vanish if 1 is taken parallel to $B$.

The splitting functions for the radiation of a gluon from this antenna are given by the matrix elements of (3.53) to Qgg final states. As in [30] and in [39], these matrix elements are given by the computation of the set of diagrams shown in Fig. 3.1. The last diagram in the figure comes from the two-gluon vertex of the operator $\bar{\sigma} \cdot F$. The third diagram is required to make the computation gauge-invariant. Its origin is most easily seen by thinking of the $Q$ as a color octet. Then this diagram is obviously an essential contribution to the radiation from the $Q g$ dipole.

With this observation, we find for the three particle matrix elements of (3.53)

$$
\begin{aligned}
& \mathcal{M}\left(Q_{L} g_{L} g_{L}\right)=\frac{1}{[b c]\left[a^{\sharp} a^{b}\right]}\left\{\frac{\left\langle A^{b} b\right\rangle}{\langle c a c]}\left(Q^{2}\left[a^{\sharp} a c\right\rangle-m^{2}\left[a^{\sharp} Q c\right\rangle\right)+\frac{\left\langle A^{b} c\right\rangle}{\langle b a b]}\left(Q^{2}\left[a^{\sharp} a b\right\rangle-m^{2}\left[a^{\sharp} Q b\right\rangle\right)\right\} \\
& \mathcal{M}\left(Q_{L} g_{R} g_{L}\right)=\frac{\left\langle a^{b} b\right\rangle\left\langle A^{b} b\right\rangle\langle b a c]}{\langle c a c]\langle b c\rangle} \\
& \mathcal{M}\left(Q_{L} g_{L} g_{R}\right)=\frac{\left\langle a^{b} c\right\rangle\left\langle A^{b} c\right\rangle\langle c a b]}{\langle b a b]\langle b c\rangle} \\
& \mathcal{M}\left(Q_{L} g_{R} g_{R}\right)=0 \\
& \mathcal{M}\left(Q_{R} g_{L} g_{L}\right)=\frac{m}{\left\langle a^{\sharp} a^{b}\right\rangle[b c]}\left\{\frac{\left\langle A^{b} b\right\rangle}{\langle c a c]}\left(\left\langle a^{\sharp} a Q c\right\rangle-Q^{2}\left\langle a^{\sharp} c\right\rangle\right)+\frac{\left\langle A^{b} c\right\rangle}{\langle b a b]}\left(\left\langle a^{\sharp} a Q b\right\rangle-Q^{2}\left\langle a^{\sharp} b\right\rangle\right)\right\} \\
& \mathcal{M}\left(Q_{R} g_{R} g_{L}\right)=\frac{m\left\langle a^{\sharp} b\right\rangle\left\langle A^{b} b\right\rangle\langle b a c]}{\left\langle a^{\sharp} a^{b}\right\rangle\langle c a c]\langle b c\rangle} \\
& \mathcal{M}\left(Q_{R} g_{L} g_{R}\right)=\frac{m\left\langle a^{\sharp} c\right\rangle\left\langle A^{b} c\right\rangle\langle c a b]}{\left\langle a^{\sharp} a^{b}\right\rangle\langle b a b]\langle b c\rangle}
\end{aligned}
$$


$\mathcal{M}\left(Q_{R} g_{R} g_{R}\right)=0$.

The case of a $Q g$ antennae in the spin $\frac{3}{2}$ state is treated similarly. The operator that defines the initial state is

$$
\left.\mathcal{O}=-\frac{i}{\sqrt{2}} \bar{Q} 1\right]\langle 2|\bar{\sigma} \cdot F| 2\rangle
$$

The two-particle matrix elements of this operator are

$$
\mathcal{M}\left(\mathcal{O} \rightarrow Q_{R} \bar{g}_{L}\right)=\left\langle 1 A^{b}\right\rangle\langle 2 B\rangle^{2}
$$

and all other matrix elements are equal to zero for the choice of 1 parallel to $B$. We will set $2=A^{b}$ and $1=B$ in the expressions that follow.

The three-particle matrix elements of (3.55) to $Q g g$ final states are

$$
\begin{aligned}
& \mathcal{M}\left(Q_{R} g_{L} \bar{g}_{L}\right)=-\frac{\left[a^{b} B\right]}{[b c]}\left\{\frac{\left\langle A^{b} b\right\rangle\left\langle A^{b}(b+c) a c\right\rangle}{\langle c a c]}+\frac{\left\langle A^{b} c\right\rangle\left\langle A^{b}(b+c) a b\right\rangle}{\langle b a b]}\right\} \\
& \mathcal{M}\left(Q_{R} g_{R} \bar{g}_{L}\right)=-\frac{\left\langle A^{b} b\right\rangle^{2}}{\langle c a c]\langle b c\rangle}\left\{\left[a^{b} c\right]\langle b Q B]+m^{2} \frac{\left\langle a^{\sharp} b\right\rangle}{\left\langle a^{\sharp} a^{b}\right\rangle}[c B]\right\} \\
& \mathcal{M}\left(Q_{R} g_{L} \bar{g}_{R}\right)=-\frac{\left\langle A^{b} c\right\rangle^{2}}{\langle b a b]\langle b c\rangle}\left\{\left[a^{b} b\right]\langle c Q B]+m^{2} \frac{\left\langle a^{\sharp} c\right\rangle}{\left\langle a^{\sharp} a^{b}\right\rangle}[b B]\right\} \\
& \mathcal{M}\left(Q_{R} g_{R} \bar{g}_{R}\right)=0 \\
& \mathcal{M}\left(Q_{L} g_{L} \bar{g}_{L}\right)=-\frac{m\left[a^{\sharp} B\right]}{\left\langle a^{\sharp} a^{b}\right\rangle[b c]}\left\{\frac{\left\langle A^{b} b\right\rangle\left\langle A^{b}(b+c) a c\right\rangle}{\langle c a c]}+\frac{\left\langle A^{b} c\right\rangle\left\langle A^{b}(b+c) a b\right\rangle}{\langle b a b]}\right\} \\
& \mathcal{M}\left(Q_{L} g_{R} \bar{g}_{L}\right)=-\frac{m\left\langle A^{b} b\right\rangle^{2}}{\left[a^{\sharp} a^{b}\right]} \frac{\left(\left[a^{\sharp} B\right]\langle b a c]+\left[a^{\sharp} c\right]\langle b c B]\right)}{\langle c a c]\langle b c\rangle} \\
& \mathcal{M}\left(Q_{L} g_{L} \bar{g}_{R}\right)=-\frac{m\left\langle A^{b} c\right\rangle^{2}}{\left[a^{\sharp} a^{b}\right]} \frac{\left(\left[a^{\sharp} B\right]\langle c a b]+\left[a^{\sharp} b\right]\langle c b B]\right)}{\langle b a b]\langle b c\rangle} \\
& \mathcal{M}\left(Q_{L} g_{R} \bar{g}_{R}\right)=0 .
\end{aligned}
$$

The splitting functions for $Q g \rightarrow Q g g$ that are derived from these expressions and those in (3.54) are catalogued in Appendix A. 
The $Q g$ antennae can also radiate by gluon splitting to a pair of quarks. For the spin $\frac{1}{2}$ case, the relevant matrix elements are

$$
\begin{aligned}
& \mathcal{M}\left(Q_{L} \bar{q}_{R} q_{L}\right)=\frac{\left\langle a^{b} b\right\rangle\left\langle A^{b} b\right\rangle}{\langle b c\rangle} \\
& \mathcal{M}\left(Q_{L} \bar{q}_{L} q_{R}\right)=-\frac{\left\langle a^{b} c\right\rangle\left\langle A^{b} c\right\rangle}{\langle b c\rangle} \\
& \mathcal{M}\left(Q_{R} \bar{q}_{R} q_{L}\right)=\frac{m\left\langle a^{\sharp} b\right\rangle\left\langle A^{b} b\right\rangle}{\left\langle a^{\sharp} a^{b}\right\rangle\langle b c\rangle} \\
& \mathcal{M}\left(Q_{R} \bar{q}_{L} q_{R}\right)=-\frac{m\left\langle a^{\sharp} c\right\rangle\left\langle A^{b} c\right\rangle}{\left\langle a^{\sharp} a^{b}\right\rangle\langle b c\rangle}
\end{aligned}
$$

For the spin $\frac{3}{2}$ case, the matrix elements are

$$
\begin{aligned}
& \mathcal{M}\left(Q_{R} \bar{q}_{R} q_{L}\right)=\frac{\left[a^{b} B\right]\left\langle A^{b} b\right\rangle^{2}}{\langle b c\rangle} \\
& \mathcal{M}\left(Q_{R} \bar{q}_{L} q_{R}\right)=-\frac{\left[a^{b} B\right]\left\langle A^{b} c\right\rangle^{2}}{\langle b c\rangle} \\
& \mathcal{M}\left(Q_{L} \bar{q}_{R} q_{L}\right)=\frac{m\left[a^{\sharp} B\right]\left\langle A^{b} b\right\rangle^{2}}{\left[a^{\sharp} a^{b}\right]\langle b c\rangle} \\
& \mathcal{M}\left(Q_{L} \bar{q}_{L} q_{R}\right)=-\frac{m\left[a^{\sharp} B\right]\left\langle A^{b} c\right\rangle^{2}}{\left[a^{\sharp} a^{b}\right]\langle b c\rangle}
\end{aligned}
$$

The splitting functions for $Q g \rightarrow Q g g$ that are derived from these expressions are catalogued in Appendix A.

\subsection{Antennae of a pair of massive particles}

After a pair of massive scalars or fermions are produced, their first emission of a gluon is described by an antenna in which the two massive particles both appear. For a complete description, we need the splitting functions for these antenna as well. These formulae are somewhat more complicated than those derived above, since some of the simplifications that are possible when the particle $b$ is massless no longer apply. 
There is little additional complexity in the cases in which the two massive particles have different masses, so we will write the formulae for that more general situation.

The case of a pair of scalars is relatively straightforward. The scalar particles themselves are spinless, so there is only one case, described by the spin- 0 operator

$$
\mathcal{O}=S_{1}^{\dagger} S_{2}
$$

The matrix element of this operator to create the state $S_{1} \bar{S}_{2}$ is simply 1 . The matrix elements for gluon emission are

$$
\begin{aligned}
& \mathcal{M}\left(S_{1} g_{L} \bar{S}_{2}\right)=\frac{1}{\left[a^{b} c\right]}\left\{\frac{\left[a^{b} a c\right\rangle}{[c a c\rangle}-\frac{\left\langle a^{b} b c\right]}{\langle c b c]}\right\} \\
& \mathcal{M}\left(S_{1} g_{R} \bar{S}_{2}\right)=-\frac{1}{\left\langle a^{b} c\right\rangle}\left\{\frac{\left\langle a^{b} a c\right]}{\langle c a c]}-\frac{\left\langle a^{b} b c\right]}{\langle c b c]}\right\} .
\end{aligned}
$$

Each expression can be brought down to one term using the Schouten identity

$$
\langle c a f]\langle d b g]-\langle d a f]\langle c b g]=-\langle c d\rangle[f a b g] .
$$

This identity is valid when $a$ and $b$ are massive vectors, possibly with different masses; $c, d, f$, and $g$ must be massless. To prove the identity, write $a$ as a linear combination of $a^{b}$ and $a^{\sharp}$. Using 3.62,

$$
\begin{aligned}
\mathcal{M}\left(S_{1} g_{L} \bar{S}_{2}\right) & =-\frac{\langle c a b c\rangle}{\langle c a c][c b c\rangle} \\
\mathcal{M}\left(S_{1} g_{L} \bar{S}_{2}\right) & =\frac{[c a b c]}{[c a c\rangle\langle c b c]}
\end{aligned}
$$

The splitting functions are readily assembled from these expressions.

For the antenna of a massive fermion and a massive scalar, the general case is described by the spin $\frac{1}{2}$ operator

$$
\left.\mathcal{O}=\bar{Q}_{1} 1\right\rangle S_{2}
$$


The two-body matrix elements of this operator are

$$
\mathcal{M}\left(Q_{1 L} \bar{S}_{2}\right)=\left\langle A^{b} 1\right\rangle
$$

and zero for $Q_{1 R}$. If we take $1=B^{\mathrm{b}}$ following the prescriptions above,

$$
\left|\left\langle A^{b} 1\right\rangle\right|^{2}=\left(E_{1}+K\right)\left(E_{2}+K\right)
$$

where $E_{1}, E_{2}$, and $K$ are the two energies and the momentum in the antenna center of mass frame.

The matrix elements for the operator (3.64) to create $Q g \bar{S}$ states is given by the expression

$$
\left.\left.\mathcal{M}=-\frac{g T^{a}}{\sqrt{2}} \bar{u}(a)\left[\frac{\phi(c)(\phi+\phi+m)}{[c a c\rangle} 1\right\rangle-1\right\rangle \frac{2 b \cdot \epsilon(c)}{[c b c\rangle}\right],
$$

where $\epsilon(c)$ is the polarization vector of the gluon. A convenient way to treat this is to manipulate

$$
\phi(c)(\phi+\phi+m)=2 a \cdot \epsilon(c)+\phi(c) \phi
$$

plus a term proportional to $(\not h-m)$ that gives zero when applied to $\bar{u}(a)$. The first term in (3.68) combines with the last term in (3.67) to give an amplitude proportional that of the scalar-scalar case, (3.61) or (3.63) above. The term with $\phi(c)$ vanishes for $g_{R}$ and gives a simple but nonzero term for $g_{L}$. The final results for the two amplitudes, after dropping the factor of $\left(g T^{a}\right)$, are

$$
\begin{aligned}
\mathcal{M}\left(Q_{L} g_{R} \bar{S}\right) & =\frac{\left\langle a^{b} 1\right\rangle\langle c a b c\rangle}{\langle c a c][c b c\rangle} \\
\mathcal{M}\left(Q_{R} g_{R} \bar{S}\right) & =\frac{m_{1}\left\langle a^{\sharp} 1\right\rangle\langle c a b c\rangle}{\left\langle a^{\sharp} a^{b}\right\rangle\langle c a c][c b c\rangle} \\
\mathcal{M}\left(Q_{L} g_{L} \bar{S}\right) & =-\frac{\left\langle a^{b} 1\right\rangle[c a b c]}{[c a c\rangle\langle c b c]}+\frac{\left\langle a^{b} c\right\rangle\langle c 1\rangle}{[c a c\rangle} \\
\mathcal{M}\left(Q_{R} g_{L} \bar{S}\right) & =-\frac{m_{1}}{\left\langle a^{\sharp} a^{b}\right\rangle}\left[\frac{\left\langle a^{\sharp} 1\right\rangle[c a b c]}{[c a c\rangle\langle c b c]}-\frac{\left\langle a^{\sharp} c\right\rangle\langle c 1\rangle}{[c a c\rangle}\right] .
\end{aligned}
$$

Here $m_{1}$ is the mass of the fermion $Q_{1}$. The formulae apply for any values of the 
masses of the fermion and scalar, as long as the 4-vectors $a$ and $b$ are properly on mass shell.

The decomposition of the gluon coupling to a massive fermion given in 3.68 is equivalent the representation of this coupling by the second-order Dirac equation, in which the fermion is replaced by a field with a scalar-type coupling and a magnetic moment coupling. The single-gluon magnetic moment coupling has a chiral structure and vanishes for specific combinations of the fermion and gluon spin. This secondorder Dirac formalism is discussed in more detail in [50].

For massive fermions, there are two cases, corresponding to total spin 0 and 1 along the antenna axis. For the spin 0 case, we could use the operator $\bar{Q}_{L} Q_{L}$ to create the antenna, similarly to the choices in Sections 2 and 5 . However, in the case in which both fermions are massive, that operator creates both $Q_{L} \bar{Q}_{L}$ and $Q_{R} \bar{Q}_{R}$ states. We will avoid that problem here by taking the operator that creates an initial state of $Q_{L} \bar{Q}_{L}$ to be

$$
\left.\mathcal{O}=\bar{Q}_{1} 1\right\rangle\left\langle 2 Q_{2}\right.
$$

The two-body matrix elements of this operator are

$$
\mathcal{M}\left(Q_{1 L} \bar{Q}_{2 L}\right)=\left\langle A^{b} 1\right\rangle\left\langle 2 B^{b}\right\rangle
$$

and zero for the other three helicity states. Similarly, for the spin 1 case, we will use the operator

$$
\left.\mathcal{O}=\bar{Q}_{1} 1\right\rangle\left[2 Q_{2}\right.
$$

to create an initial state of $Q_{L} \bar{Q}_{R}$. The two-body matrix elements of this operator are

$$
\mathcal{M}\left(Q_{1 L} \bar{Q}_{2 R}\right)=\left\langle A^{b} 1\right\rangle\left[2 B^{b}\right]
$$

and zero for the other three helicity states. The $Q g \bar{Q}$ matrix elements of these operators are easily computed using the methods presented earlier in this section. The results for the splitting functions are tabulated in Appendix A. 


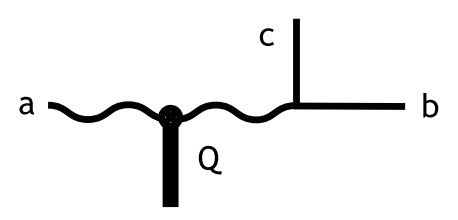

Figure 3.2: The single Feynman diagram for the computation of the $g g \rightarrow g F \bar{F}$ splitting function [39].

\subsection{Antennae with massive particle production}

There is one more situation that we must consider. At very high energies, massive particles can be produced by gluon splitting. At the LHC, for example, parton-parton scattering can give quark-gluon and gluon-gluon collisions with center of mass energies well above $1 \mathrm{TeV}$. Final state gluon antennae in these collsions can produce pairs of top quarks. The pair production amplitudes are relatively simple, since each requires only one Feynman diagram, as shown in Fig. 3.2 for the $g g \rightarrow g \bar{t} t$ case. The final pair of heavy particles must have equal mass and equal spin. However, there are a large number of cases to enumerate. The massive scalar or fermion pair can be formed from a spin $\frac{1}{2}$ or a spin $\frac{3}{2} q g$ antenna or from a spin 0 or spin $2 g g$ antenna.

For scalar pair production, the formalism is actually quite simple. The spin $\frac{1}{2}$ and spin $\frac{3}{2} q g$ antennae can be represented by the operators

$$
\begin{aligned}
\mathcal{O}_{1 / 2} & =-\frac{i}{\sqrt{2}} \bar{q} \bar{\sigma} \cdot F|2\rangle \\
\mathcal{O}_{3 / 2} & \left.=-\frac{i}{\sqrt{2}} \bar{q} 1\right]\langle 2|\bar{\sigma} \cdot F| 2\rangle .
\end{aligned}
$$

If the gluon splits to a pair of scalars, both cases involve the operator $\bar{\sigma} \cdot F$ dotted with the $g S \bar{S}$ vertex. This product is

$$
\frac{1}{2}\left[(\not b+\phi) \gamma^{\mu}-\gamma^{\mu}(\not b+\phi)\right](b-c)_{\mu} \equiv[b, c]
$$


so that the relevant three-particle matrix elements are

$$
\mathcal{M}\left(q_{L} \bar{S} S\right)=-\frac{\langle a[b, c] 2\rangle}{s_{b c}}
$$

for the spin $\frac{1}{2}$ case and

$$
\mathcal{M}\left(q_{R} \bar{S} S\right)=\frac{[a 1]\langle 2[b, c] 2\rangle}{s_{b c}}
$$

for the spin $\frac{3}{2}$ case.

Similarly, the spin 0 and spin $2 g g$ antennae, corresponding to the $g_{L} g_{L}$ and $g_{R} g_{L}$ initial states, can be represented by the operators

$$
\begin{aligned}
& \mathcal{O}_{0}=\frac{1}{2} \operatorname{tr}\left[(\bar{\sigma} \cdot F)^{2}\right] \\
& \mathcal{O}_{2}=[1|\sigma \cdot F| 1]\langle 2|\bar{\sigma} \cdot F| 2\rangle .
\end{aligned}
$$

The manipulation (3.75) again gives a simple form for the three-particle matrix elements. The corresponding splitting functions are given in Appendix A.

For the case of massive fermion pair production, this formalism is necessarily more complex. With the choice of helicity states that we have used throughout this paper, the vertex to create a pair of massive fermions is a Dirac matrix. For the case of a final-state $\bar{Q}_{R} Q_{L}$, for example, this matrix has the form

$$
\left.V=c^{b}\right]\left\langle b^{b}-\frac{m^{2}}{\left\langle c^{\sharp} c^{b}\right\rangle\left[b^{b} b^{\sharp}\right]} b^{\sharp}\right]\left\langle c^{\sharp}\right.
$$

Then the matrix element of $\bar{\sigma} \cdot F$ contains the structure

$$
\left\langle R_{1}[(b+c), V] R_{2}\right\rangle
$$

with a commutator bracketed between reference vectors $R_{1}$ and $R_{2}$. However, the frame-dependent choice of the vectors $b^{\sharp}, c^{\sharp}$ makes it difficult to simplify this expression further. It is true that $(b+c)=Q-a$, where $a$ is now massless. In some cases, we have $R_{1}=a$, in which case the $a$ term cancels. In other cases, we have $R_{1}=2=A^{b}$, so that the $a$ term vanishes if $a$ is collinear with $A$. We list the full expressions for 
these splitting functions in Appendix A.

\subsection{Conclusion}

In this paper, we have provided new materials for the construction of parton showers that include massive spin 0 and spin $\frac{1}{2}$ particles. We hope that this formalism we have presented will be useful in describing the QCD dynamics of the top quark and other heavy particles at LHC. 


\section{Part II}

New Observables for Jet Structure 


\section{Chapter 4}

\section{Angular Scaling in Jets}

\subsection{Introduction}

Over the past years, experimental and theoretical advances have made it possible to ask increasingly detailed questions about jets. Recently, there has been considerable interest in jet substructure [10-12]. Much work has focused on identifying jets initiated by boosted heavy objects such as the Higgs boson and the top quark. A variety of techniques have been proposed to identify and characterize substructure on a jet-by-jet basis. It is natural to draw upon these techniques to motivate interesting observables for the study of QCD. In this paper we explore an observable defined on ensembles of jets.

A natural candidate for such an observable is the ensemble average of the angular structure function introduced in Ref. [13]. We will see that this ensemble average has a clear physical interpretation in terms of an average scaling exponent. Its leading order behavior can be found from a napkin-sized computation. Below we will argue that this ensemble average provides an interesting observable for at least three different reasons. First, it is an infrared and collinear safe observable that, in the perturbative regime, measures the extent to which QCD jet dynamics is scale invariant. Second, because it is formulated in terms of two-particle correlations, it asks a particularly detailed question about jet substructure. We find that different Monte Carlo event 
generators give significantly different predictions. Consequently measuring this ensemble average could give valuable feedback on the performance of the Monte Carlo, with any disagreements pointing towards the need for additional tuning or improvement of the physics modeling. Finally, the ensemble average has a simple dependence on uncorrelated radiation, such as might be expected from underlying event (UE) or pile-up (PU) contributions to jets. This suggests that measurements of the ensemble average could yield useful information about the average contribution of the underlying event and pile-up to hard perturbative jets. More broadly, contributions to the ensemble average with different scaling behaviors will be more or less important at smaller or larger angular scales. In this sense the ensemble average exhibits a characteristic sensitivity to both perturbative and non-perturbative physics, with the former dominating at small angular scales and the latter becoming important at large angular scales.

The outline of this paper is as follows. In Sec. 4.2 we review the definition of the angular structure function and introduce its ensemble average. In Sec. 4.3 we compute the leading order behavior of the ensemble average in the collinear approximation and discuss expectations for corrections to the leading order result. In Sec. 4.4 we investigate the sensitivity of the ensemble average to the underlying event and pile-up, formulating a procedure for measuring the average density of uncorrelated radiation for a given ensemble of jets. In Sec. 4.5 we gain additional insight into the physics of the ensemble average by considering ensembles of soft radiation in the transverse regions of the detector. In Sec. 4.6 we discuss our results and present our conclusions.

\subsection{Average angular structure function}

It has long been appreciated that jets have a fractal-like structure. This point of view emerges naturally from the description of the parton shower as a probabilistic Markov chain. The authors of Ref. [51] have computed the fractal dimension of a jet, while Ref. [52] advocates the use of the fractal phase space introduced in Ref. [53] as a useful diagnostic tool for complex events.

Correlation functions provide a convenient language for studying fractal systems. 
In particular they can be used to define fractal dimensions through their limiting behavior at small scales. With this in mind, let us review the pair of correlation functions introduced in Ref. [13]. The first is the 'angular correlation function,' defined as 1

$$
\mathcal{G}(R) \equiv \sum_{i \neq j} p_{T i} p_{T j} \Delta R_{i j}^{2} \Theta\left(R-\Delta R_{i j}\right)
$$

where the sum runs over all pairs of constituents of a given jet and $\Theta(x)$ is the Heaviside step function. Here $p_{T i}$ is the transverse momentum of constituent $i$, and $\Delta R_{i j}$ is the Euclidean distance between $i$ and $j$ in the pseudorapidity $(\eta)$ and azimuthal angle $(\phi)$ plane: $\Delta R_{i j}^{2}=\left(\eta_{i}-\eta_{j}\right)^{2}+\left(\phi_{i}-\phi_{j}\right)^{2}$. Infrared and collinear safety and $z$-boost invariance fix this as the unique form for a two-particle angular correlation function defined on the constituents of a jet, with the only remaining freedom being that the exponent of $\Delta R_{i j}$ in Eq. 4.1 is arbitrary so long as it is positive. $\mathcal{G}(R)$ is the contribution to a jet's mass from constituents separated by an angular distance of $R$ or less. It is worth emphasizing that $R$ does not mark the distance with respect to any fixed center.

In the context of fractals, a correlation function $c(R)$ gives rise to a corresponding correlation dimension $\mathcal{D}_{c}$ defined as [54]:

$$
\mathcal{D}_{c} \equiv \lim _{R \rightarrow 0} \frac{\log c(R)}{\log R}
$$

There is of course an immediate obstacle to using Eq. 4.1 and Eq. 4.2 to define the correlation dimension of a jet: the finite resolution of the detector makes the small $R$ limit inaccessible. In addition, the fractal-like structure of a jet does not continue down to arbitrarily small scales, since, for a jet with transverse momentum $p_{T}$, the parton shower is cutoff at an angular scale $R_{\min } \gtrsim \Lambda_{\mathrm{QCD}} / p_{T}$. A sensible alternative to Eq. 4.2 is to instead define an 'angular structure function' $\Delta \mathcal{G}(R)$ via a logarithmic

\footnotetext{
${ }^{1}$ In Ref. $13 \mathcal{G}(R)$ is normalized so that $\mathcal{G}(R) \rightarrow 1$ at large $R$; however, for the purposes of this paper it is convenient to leave $\mathcal{G}(R)$ unnormalized with dimensions of mass squared.
} 
derivative:

$$
\Delta \mathcal{G} \equiv \frac{d \log \mathcal{G}}{d \log R}=\frac{R}{\mathcal{G}} \frac{d \mathcal{G}}{d R}=R \frac{\sum_{i \neq j} p_{T i} p_{T j} \Delta R_{i j}^{2} \delta\left(R-\Delta R_{i j}\right)}{\sum_{i \neq j} p_{T i} p_{T j} \Delta R_{i j}^{2} \Theta\left(R-\Delta R_{i j}\right)}
$$

For a jet with a finite number of constituents, the $\delta$-function in Eq. 4.3 results in a noisy function of $R$. A convenient way to obtain a smooth version of $\Delta \mathcal{G}(R)$ is to replace the $\delta$-function by a gaussian with a fixed width $d R$ :

$$
\delta(x) \rightarrow \delta_{\mathrm{dR}}(x)=\frac{\exp \left(-x^{2} / 2 d R^{2}\right)}{\sqrt{2 \pi} d R}
$$

In order to maintain the leftmost equivalence in Eq. 4.3 the $\Theta$-function must also be replaced by an error function $\Theta_{\mathrm{dR}}$ with the same width $d R$.

The angular structure function $\Delta \mathcal{G}(R)$ encodes the scaling of the angular correlation function at a particular value of $R$. In particular if $\mathcal{G}(R) \sim R^{\beta}$ then $\Delta \mathcal{G}(R)=\beta$. In this sense $\Delta \mathcal{G}(R)$ recovers a scaling exponent analogous to the correlation dimension in Eq. 4.2. On a jet-by-jet basis $\Delta \mathcal{G}(R)$ exhibits dramatic peaks at prominent angular scales corresponding to separations between hard substructure in the jet. This property of $\Delta \mathcal{G}(R)$ is exploited in Ref. [13] to construct an efficient top tagging algorithm. In order to clearly observe scaling exponents, however, we will need to average over large ensembles of jets, since the number of final state particles in a single jet is too few to clearly observe fractal structure. Such an ensemble average will be the subject of the rest of this paper.

We use the angular correlation function as our basic object, defining its ensemble average as

$$
\langle\mathcal{G}(R)\rangle \equiv \frac{1}{N} \sum_{k=1}^{N} \mathcal{G}(R)_{k}
$$

where $N$ is the size of the ensemble and $\mathcal{G}(R)_{k}$ is the angular correlation function of the $k$ th jet. From this average, we define the average angular structure function:

$$
\langle\Delta \mathcal{G}(R)\rangle \equiv \frac{R}{\langle\mathcal{G}(R)\rangle} \frac{d}{d R}\langle\mathcal{G}(R)\rangle
$$




$$
\begin{aligned}
& =R \frac{\sum_{k=1}^{N} \mathcal{G}^{\prime}(R)_{k}}{\sum_{k=1}^{N} \mathcal{G}(R)_{k}} \\
& =R \frac{\sum_{k=1}^{N} \sum_{i \neq j} p_{T k, i} p_{T k, j} \Delta R_{i j}^{2} \delta_{\mathrm{dR}}\left(R-\Delta R_{i j}\right)}{\sum_{k=1}^{N} \sum_{i \neq j} p_{T k, i} p_{T k, j} \Delta R_{i j}^{2} \Theta_{\mathrm{dR}}\left(R-\Delta R_{i j}\right)}
\end{aligned}
$$

where $\delta_{\mathrm{dR}}(R)$ and $\Theta_{\mathrm{dR}}(R)$ are the gaussian and error functions with width $d R$, respectively. Note that the ensemble average is not defined as an average over $N$ angular structure functions $\Delta \mathcal{G}(R)_{k}$ :

$$
\langle\Delta \mathcal{G}(R)\rangle \neq \frac{1}{N} \sum_{k=1}^{N} \Delta \mathcal{G}(R)_{k}
$$

We make this choice for at least two reasons. First, the definition in Eq. 4.6 lends itself more easily to analytical computation and makes possible its interpretation as an average scaling exponent. Second, on an event-by-event basis, the angular structure function is quite noisy. Consequently, the ensemble average in Eq. 4.7 is significantly noisier than that in Eq. 4.6 .

Throughout this paper we will set $d R=0.04$. Although nonzero $d R$ sculpts the ensemble averages somewhat, especially near $R=0$, for $d R=0.04$ the effect is small enough that we need not consider it when calculating $\langle\mathcal{G}(R)\rangle$ analytically.

\subsection{Calculating the average}

As we will now show, a striking property of $\langle\Delta \mathcal{G}(R)\rangle$ is that its leading order behavior can be understood from a simple computation. This is in contrast to, e.g., the integrated jet shape $\Psi(R)$ [55], which requires a detailed calculation even for

its leading order behavior [56]. This section is organized as follows. In Sec. 4.3.1 we compute the leading order behavior of $\langle\Delta \mathcal{G}(R)\rangle$ in the collinear approximation. While it would be rewarding to perform a NLO computation of $\langle\Delta \mathcal{G}(R)\rangle$, in the subsequent sections we limit ourselves to exploring some of the features we expect to emerge from a more complete calculation. This task will be made easier thanks to the 
clear physical interpretation of $\langle\Delta \mathcal{G}(R)\rangle$ as a scaling exponent. First, in Sec. 4.3 .2 we discuss the qualitative effect of the running of the strong coupling. Second, in Sec. 4.3 .3 we explore higher order effects with an emphasis on the expected difference between quark and gluon jets. Finally, in Sec. 4.3.4 we briefly touch upon whether $\langle\Delta \mathcal{G}(R)\rangle$ could be amenable to factorization.

\subsubsection{Collinear approximation}

To begin we compute the average value of the angular correlation function $\langle\mathcal{G}(R)\rangle$ in the collinear approximation. To first order in $\alpha_{s},\langle\mathcal{G}(R)\rangle$ can be computed from

$$
\langle\mathcal{G}(R)\rangle \simeq \frac{\alpha_{s}}{2 \pi} p_{T}^{2} \int_{0}^{R_{0}^{2}} \frac{d \theta^{2}}{\theta^{2}} \int_{0}^{1} d z P(z) z(1-z) \theta^{2} \Theta(R-\theta)
$$

where $R_{0}$ is the radius of the jet algorithm and $P(z)$ is the appropriate Altarelli-Parisi splitting function. Notice that, as discussed at the end of Sec. 4.2 , for the purposes of this section it is enough to set $d R=0$, although $d R>0$ will be needed for any actual measurement. Since we are interested in the interior of the jet, in all of the following expressions we will assume that $R<R_{0}$. This prevents us from making predictions about edge effects in $\langle\mathcal{G}(R)\rangle$, but we do not expect the collinear approximation to be a good approximation at larger $R$ anyway. From Eq. 4.8 we find:

$$
\langle\mathcal{G}(R)\rangle=\frac{\alpha_{s}}{2 \pi} p_{T}^{2} R^{2} \begin{cases}\frac{3}{4} C_{F} & \text { quark jets } \\ \frac{7}{10} C_{A}+\frac{1}{10} n_{F} T_{R} & \text { gluon jets }\end{cases}
$$

We thus have the leading order result that the angular correlation function for QCD jets goes like $R^{2}$. Consequently, for both quark and gluon jets, we have that $\langle\Delta \mathcal{G}(R)\rangle=2$. Note that this holds for any jet algorithm.

Some interpretation of this result is in order. The average angular structure function $\langle\Delta \mathcal{G}(R)\rangle$ is a measure of how energy is distributed within a jet. A typical QCD jet has a hard core with the structure of emissions in and around the core controlled by the soft and collinear singularities. The fact that for QCD jets $\langle\Delta \mathcal{G}(R)\rangle=2$ at leading order tells us that QCD has a collinear singularity of strength $d \Theta^{2} / \Theta^{2}$. By 
contrast, if the energy were distributed uniformly over the entire cone of the jet, we would expect $\langle\mathcal{G}(R)\rangle \sim R^{4}$ and $\langle\Delta \mathcal{G}(R)\rangle \simeq 4$, at least up to edge effects.

\subsubsection{Running coupling}

The qualitative effects of including a running coupling are straightforward to understand. We proceed by evaluating the strong coupling at a characteristic energy scale $Q^{2}=p_{T}^{2} \theta^{2} g(z)$, where $g(z)$ is a function of the momentum fraction $z$ that is regular for $0 \leq z \leq 1$. Typically, $Q$ is taken to be the jet mass $(g(z)=z(1-z))$ or transverse momentum of the emitted gluon $(g(z)=1-z)$, but for our purposes what is most important is that $Q^{2}$ is proportional to $\theta^{2}$. This implies that at small angles the strong coupling increases resulting in more radiation in the small angle region of

phase space. Effectively, this increases the strength of the collinear singularity with respect to the fixed coupling expectation. Thus, we expect that the effect of a running coupling is to lower the value of $\langle\Delta \mathcal{G}(R)\rangle$ with respect to the fixed coupling result.

A simple calculation confirms this picture. Now including a running coupling we have:

$$
\langle\mathcal{G}(R)\rangle \simeq p_{T}^{2} \int \frac{d \theta^{2}}{\theta^{2}} \int d z P(z) z(1-z) \frac{\alpha_{s}\left(p_{T}^{2} \theta^{2} g(z)\right)}{2 \pi} \theta^{2} \Theta(R-\theta)
$$

To lowest order the running coupling is

$$
\alpha_{s}\left(Q^{2}\right)=\frac{\alpha_{0}}{\log \left(\frac{p_{T}^{2} \theta^{2} g(z)}{\Lambda_{\mathrm{QCD}}^{2}}\right)}
$$

where $\alpha_{0}=2 \pi / \beta_{0}$ and $\beta_{0}$ is the leading coefficient of the QCD beta function. In the limit that $p_{T} R \gg \Lambda_{\mathrm{QCD}}$, the precise forms of $g(z)$ and $P(z)$ are irrelevant, and we find that

$$
\langle\mathcal{G}(R)\rangle \propto \frac{R^{2}}{\log \left(\frac{p_{T} R}{\Lambda_{\mathrm{QCD}}}\right)}
$$

with the result that

$$
\langle\Delta \mathcal{G}(R)\rangle \simeq 2-\frac{1}{\log \left(\frac{p_{T} R}{\Lambda_{\mathrm{QCD}}}\right)}
$$


As expected, including the running coupling decreases the average angular structure function. To first order in $1 / \log \left(\frac{p_{T} R}{\Lambda_{\mathrm{QCD}}}\right)$, Eq. 4.13 is true for both quark and gluon jets. This effect is not negligible. For example, for $p_{T}=200 \mathrm{GeV}, R=1.0$, and $\Lambda_{\mathrm{QCD}}=300 \mathrm{MeV}$, we have that $1 / \log \left(\frac{p_{T} R}{\Lambda_{\mathrm{QCD}}}\right) \simeq 0.15$.

\subsubsection{Higher order effects}

We can get an idea of the nature of the higher order corrections to the $\mathcal{O}\left(\alpha_{s}\right)$ fixed coupling result by continuing the calculation of Sec. 4.3.1 to $\mathcal{O}\left(\alpha_{s}^{2}\right)$. The kinematic identifications of the three parton final state are illustrated in Fig. 4.1, which depicts the longitudinal momentum fractions and angles associated to each splitting. We impose angular ordering so that $\theta_{1} \geq \theta_{2}$. The expression for the $\mathcal{O}\left(\alpha_{s}^{2}\right)$ contribution to $\langle\mathcal{G}(R)\rangle$ is then given by:

$$
\begin{aligned}
\langle\mathcal{G}(R)\rangle_{\alpha_{s}^{2}} \simeq & \left(\frac{\alpha_{s}}{2 \pi}\right)^{2} p_{T}^{2} \int_{0}^{R_{0}^{2}} \frac{d \theta_{1}^{2}}{\theta_{1}^{2}} \int_{0}^{\theta_{1}^{2}} \frac{d \theta_{2}^{2}}{\theta_{2}^{2}} \int_{0}^{1} d z \int_{0}^{1} d y P_{1}(z) P_{2}(y) \\
& \left\{z^{2} y(1-y) \theta_{2}^{2} \Theta\left(R-\theta_{2}\right)\right. \\
+ & z(1-z) y \int_{0}^{2 \pi} \frac{d \phi}{2 \pi}\left[\left((1-y)^{2} \theta_{2}^{2}+\theta_{1}^{2}-2(1-y) \theta_{1} \theta_{2} \cos \phi\right)\right. \\
& \times \Theta\left(R-\sqrt{\left.(1-y)^{2} \theta_{2}^{2}+\theta_{1}^{2}-2(1-y) \theta_{1} \theta_{2} \cos \phi\right)}\right] \\
+ & z(1-z)(1-y) \int_{0}^{2 \pi} \frac{d \phi}{2 \pi}\left[\left(\left(y^{2} \theta_{2}^{2}+\theta_{1}^{2}+2 y \theta_{1} \theta_{2} \cos \phi\right)\right.\right. \\
& \left.\left.\times \Theta\left(R-\sqrt{y^{2} \theta_{2}^{2}+\theta_{1}^{2}+2 y \theta_{1} \theta_{2} \cos \phi}\right)\right]\right\}
\end{aligned}
$$

Using unpolarized splitting functions and setting $\cos \phi=0$ results in a simple analytic formula given by

$\langle\mathcal{G}(R)\rangle_{q}=\frac{\alpha_{s}}{2 \pi} p_{T}^{2} R^{2}\left\{\frac{3}{4} C_{F}+\frac{\alpha_{s}}{2 \pi}\left(-\frac{25}{16} C_{F}^{2}+\frac{49}{120} C_{A} C_{F}+\frac{7}{120} n_{F} T_{R} C_{F}\right)\left(1+\log \frac{R_{0}^{2}}{R^{2}}\right)\right\}$

for quark jets and

$$
\langle\mathcal{G}(R)\rangle_{g}=\frac{\alpha_{s}}{2 \pi} p_{T}^{2} R^{2}\left\{\frac{7}{10} C_{A}+\frac{1}{10} n_{F} T_{R}\right.
$$




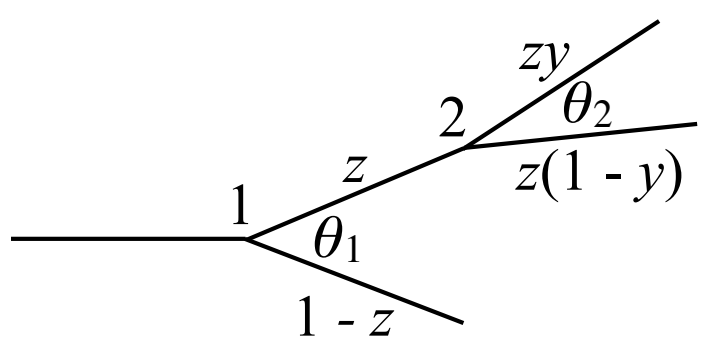

Figure 4.1: Splitting diagram for the $\mathcal{O}\left(\alpha_{s}^{2}\right)$ contribution to $\langle\mathcal{G}(R)\rangle$.

$$
\left.+\frac{\alpha_{s}}{2 \pi}\left(-\frac{49}{100} C_{A}^{2}-\frac{91}{300} C_{A} n_{F} T_{R}-\frac{1}{30} n_{F}^{2} T_{R}^{2}+\frac{7}{20} C_{F} n_{F} T_{R}\right)\left(1+\log \frac{R_{0}^{2}}{R^{2}}\right)\right\}
$$

for gluon jets. From these expressions we can calculate $\langle\Delta \mathcal{G}(R)\rangle$ to $\mathcal{O}\left(\alpha_{s}\right)$, finding

$$
\begin{aligned}
\langle\Delta \mathcal{G}(R)\rangle_{q} & \simeq 2-\frac{\alpha_{s}}{\pi}\left(-\frac{25}{12} C_{F}+\frac{49}{90} C_{A}+\frac{7}{90} n_{F} T_{R}\right)+\mathcal{O}\left(\alpha_{s}^{2}\right) \\
& \simeq 2+0.95 \frac{\alpha_{s}}{\pi}
\end{aligned}
$$

for quark jets and

$$
\begin{aligned}
\langle\Delta \mathcal{G}(R)\rangle_{g} & \simeq 2-\frac{\alpha_{s}}{\pi}\left(\frac{-\frac{49}{10} C_{A}^{2}-\frac{91}{30} C_{A} n_{F} T_{R}-\frac{1}{3} n_{F}^{2} T_{R}^{2}+\frac{7}{2} C_{F} n_{F} T_{R}}{7 C_{A}+n_{F} T_{R}}\right)+\mathcal{O}\left(\alpha_{s}^{2}\right) \\
& \simeq 2+2.44 \frac{\alpha_{s}}{\pi}
\end{aligned}
$$

for gluon jets, where we have set $n_{F}=5$ in evaluating the color factors. Notice that, since

$$
\frac{d}{d \log R} \log \left[R^{2}\left(1+\epsilon \log \left(R^{2}\right)\right)\right]=2+2 \epsilon+\mathcal{O}\left(\epsilon^{2}\right)
$$

$\langle\Delta \mathcal{G}(R)\rangle_{q / g}$ remain flat in $R$ to this order in $\alpha_{s}$. In particular Eq. 4.19 implies that the $\alpha_{s}^{2} R^{2}$ terms in Eq. 4.15 and Eq. 4.16 do not contribute to Eq. 4.17 and Eq. 4.18 at $\mathcal{O}\left(\alpha_{s}\right)$. These terms do contribute to the normalization of the angular correlation function, but we do not expect them to be correctly given by the collinear approximation. We suspect, however, that the $R^{2} \log \frac{R_{0}^{2}}{R^{2}}$ terms are robust as far as the scaling exponent is concerned. 


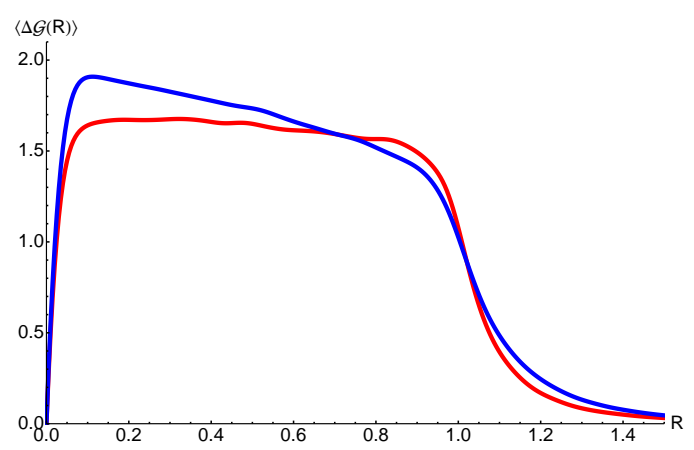

(a) Pythia8

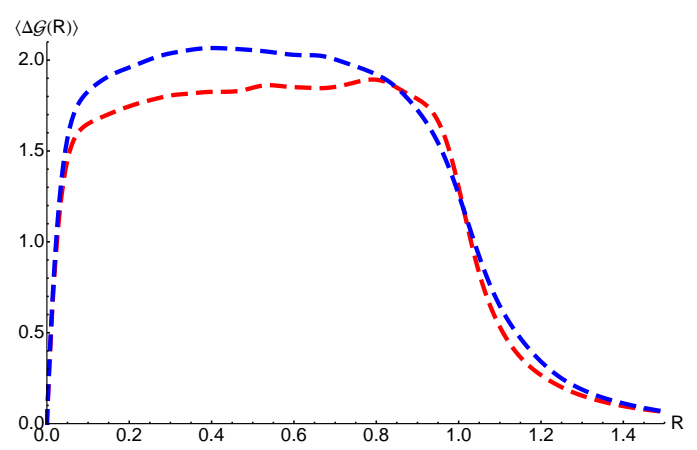

(b) Herwig ++

Figure 4.2: Average angular structure functions for ensembles of jets with $p_{T}>200 \mathrm{GeV}$ and no underlying event or initial state radiation. Red curves denote quark jets and blue curves denote gluon jets. These are anti-kT jets with jet radius $R_{0}=1.0$ as generated with Pythia8 (left) and Herwig++ (right). See Appendix C for more details about the Monte Carlo.

The increase of $\langle\Delta \mathcal{G}(R)\rangle$ with respect to the leading order result can be loosely interpreted as resulting from a relative increase in the amount of energy radiated away from the center of the jet. This increase is largest for gluon jets as a result of the large associated color factors.

Putting together the results from Sec. 4.3.1, Sec. 4.3.2, and Sec. 4.3.3 our expectations for the form of $\langle\Delta \mathcal{G}(R)\rangle$ for quark and gluon jets are as follows. Apart from edge effects, we expect both $\langle\Delta \mathcal{G}(R)\rangle_{q}$ and $\langle\Delta \mathcal{G}(R)\rangle_{g}$ to be approximately flat in $R$ as in the leading order result. Furthermore, if the effects of a running coupling are dominant then we also expect that $\langle\Delta \mathcal{G}(R)\rangle_{q / g} \lesssim 2$. In addition we expect that $\langle\Delta \mathcal{G}(R)\rangle_{q}\left\langle\langle\Delta \mathcal{G}(R)\rangle_{g}\right.$. Note that, even in the case when $\langle\Delta \mathcal{G}(R)\rangle_{q / g}$ are separately flat, an ensemble average over an admixture of quark and glue jets will in general not be flat.

In Fig. 4.2 and Fig. 4.3 we plot $\langle\Delta \mathcal{G}(R)\rangle$ for both quark and gluon jets as obtained from Pythia8 and Herwig ++ dijet events. The ensembles are composed of anti-kT jets with $R_{0}=1.0$ and $p_{T}>200 \mathrm{GeV}$. Initial state radiation (ISR) and the underlying event have been turned off. The Monte Carlo is roughly in accord with our expectations. Not surprisingly, our expectations are more in line with the angular ordered shower in Herwig ++ , where the expected difference between quark 


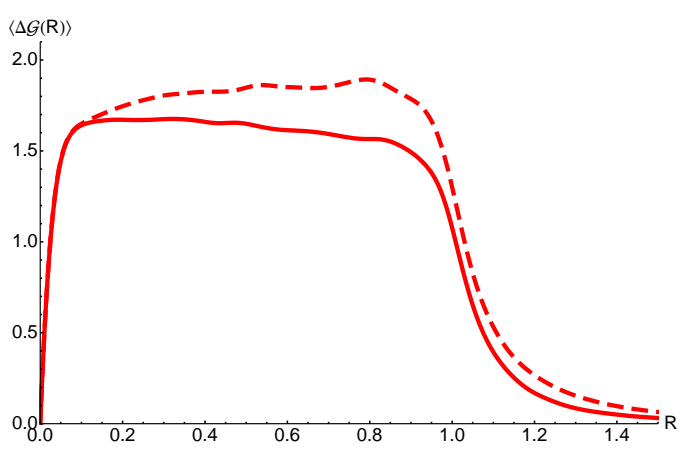

(a) Quark jets

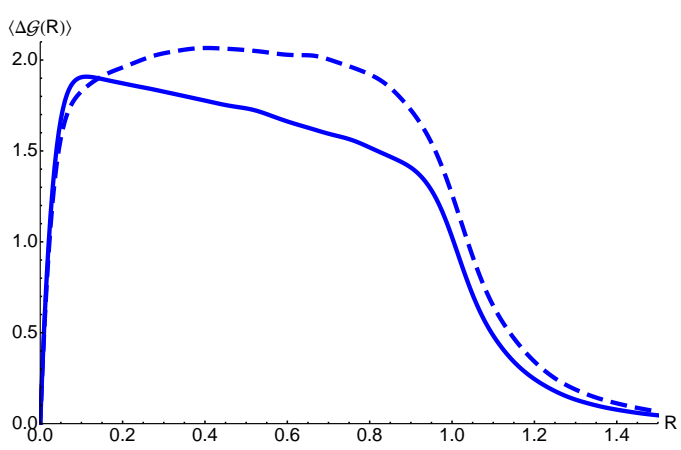

(b) Gluon jets

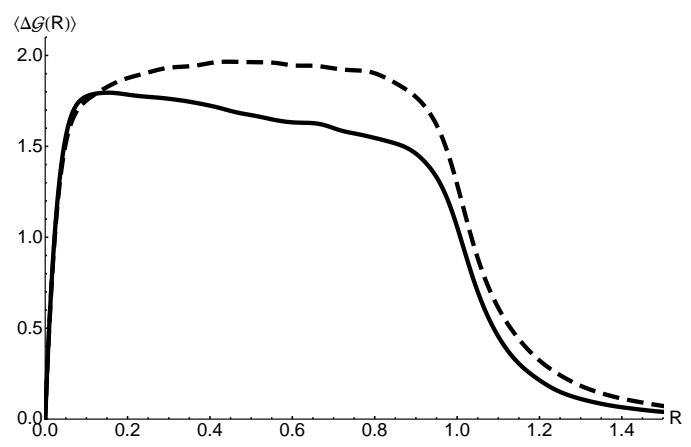

(c) Jets

Figure 4.3: Average angular structure functions for three different ensembles of jets with $p_{T}>200 \mathrm{GeV}$ and no underlying event or initial state radiation. These are anti-kT jets with jet radius $R_{0}=1.0$ as generated with Pythia8 (solid) and Herwig ++ (dashed). See Appendix $\mathrm{C}$ for more details about the Monte Carlo.

jets and glue jets, i.e. $\langle\Delta \mathcal{G}(R)\rangle_{q}<\langle\Delta \mathcal{G}(R)\rangle_{g}$, is unambiguous. The differences between the predictions for $\langle\Delta \mathcal{G}(R)\rangle$ given by Pythia8 and Herwig ++ are striking (see Fig. 4.3), with $\langle\Delta \mathcal{G}(R)\rangle$ being substantially higher for Herwig++ over a large range in $R$. This difference ${ }^{2}$, which persists when the underlying event and initial state radiation are included (see Fig. 4.4(c)), should be measurable and motivates making the measurement. It would be interesting to have a detailed understanding of how this qualitatively different behavior emerges from the two codes; doing so, however, lies outside the scope of this paper.

${ }^{2}$ We have also performed Monte Carlo calculations of $\langle\Delta \mathcal{G}(R)\rangle$ for an ensemble of jets produced in the process $e^{+} e^{-} \rightarrow q \bar{q}$. In this case the differences between Pythia8, Herwig ++ , and Pythia8+Vincia (showering done with Vincia 1.0.26 [57]) are small compared to the differences in Fig. $4.3(\mathrm{a})$ 


\subsubsection{Factorization}

Before moving on we would like to draw a connection to a recent analysis on the factorizability of jet substructure observables in soft-collinear effective theory (SCET) [58]. Walsh and Zuberi determined necessary conditions for jet substructure observables to be factorizable in the sense that any such observable can be computed as a direct product of universal terms up to power corrections. A central result of their analysis is that in order for factorization to hold an observable must not demand that soft modes individually resolve collinear modes. Because the angular correlation function is a two-particle correlation function, one might worry that it mixes soft and collinear modes in a way that upsets factorization. However, to leading power soft modes contributing to $\langle\mathcal{G}(R)\rangle$ do not resolve individual collinear modes:

$$
\sum_{C, S} p_{T C} p_{T S} \Delta R_{C S}^{2} \Theta\left(R-\Delta R_{C S}\right)=p_{T \mathrm{~J}} \sum_{S} p_{T S} \Delta R_{S \mathrm{~J}}^{2} \Theta\left(R-\Delta R_{S \mathrm{~J}}\right)
$$

Here the sum runs over collinear $(C)$ and soft $(S)$ modes and $J$ refers to the jet. This suggests that the ensemble average of the angular correlation function should be factorizable in SCET.

\subsection{Effect of uncorrelated radiation}

In the previous section we discussed the shape of $\langle\Delta \mathcal{G}(R)\rangle$ as determined by the perturbative final state shower. At a hadron collider the colored initial state means that the dynamics of jets cannot be understood separately from the underlying event

and initial state radiation. For convenience in the following we will often collectively refer to any radiation that is not associated with the hard, perturbative final state as the "underlying event." In particular what we have in mind is comparably soft radiation that is uncorrelated with the hard scatter and which is approximately uniformly distributed in pseudorapidity and azimuth with transverse momentum density $\Lambda_{\mathrm{UE}}$. 


\subsubsection{Background}

We would like to ask how this UE affects $\langle\Delta \mathcal{G}(R)\rangle$ for an ensemble of jets of a given $p_{T}$. Since by assumption $\Lambda_{\mathrm{UE}} \ll p_{T}$, we can neglect correlations of order $\Lambda_{\mathrm{UE}}^{2}$. Furthermore, since most of the energy of the final state shower is localized in a hard core at the center of the jet, we can write the $\mathcal{O}\left(\Lambda_{\mathrm{UE}}\right)$ contribution to $\langle\mathcal{G}(R)\rangle$ as

$$
\langle\mathcal{G}(R)\rangle_{\Lambda_{\mathrm{UE}}}=p_{T} \Lambda_{\mathrm{UE}} \int_{0}^{2 \pi} d \phi \int_{0}^{R} R^{\prime} d R^{\prime} R^{\prime 2}=\frac{\pi}{2} p_{T} \Lambda_{\mathrm{UE}} R^{4}
$$

This ansatz neglects edge effects due to the finite size of the jet, but these are expected to be small away from $R=R_{0}$ for the approximately circular ${ }^{3}$ anti-kT jets used throughout this paper. The range of $R$ for which this ansatz is valid will be smaller for other sequential jet algorithms (e.g. Cambridge/Aachen and $k_{T}$ ) that yield more irregularly shaped jets. Thus we have:

$$
\langle\mathcal{G}(R)\rangle=\langle\mathcal{G}(R)\rangle_{\mathrm{pert}}+\frac{\pi}{2} p_{T} \Lambda_{\mathrm{UE}} R^{4}
$$

Since the perturbative piece goes approximately like $R^{2}$, at large $R$ the UE contribution is increasingly important. In the absence of the perturbative piece we would have $\langle\Delta \mathcal{G}(R)\rangle \simeq 4$. Thus the inclusion of UE has the effect of increasing $\langle\Delta \mathcal{G}(R)\rangle$ from its perturbative value of near 2 at small $R$ towards the value of 4 characteristic of uniform radiation at large $R$. This behavior is evident in Fig. 4.4. Going to the average angular structure function, we can rewrite Eq. 4.22 as:

$$
\langle\Delta \mathcal{G}(R)\rangle_{\mathrm{pert}}=\frac{R \frac{d}{d R}\langle\mathcal{G}(R)\rangle-2 \pi p_{T} \Lambda_{\mathrm{UE}} R^{4}}{\langle\mathcal{G}(R)\rangle-\frac{\pi}{2} p_{T} \Lambda_{\mathrm{UE}} R^{4}}
$$

Provided that we have some sort of estimate of the perturbative piece $\langle\Delta \mathcal{G}(R)\rangle_{\text {pert }}$, the distinctive $R$-dependence of Eq. 4.23 can be used to fit for $\Lambda_{\mathrm{UE}}$. In the remainder of this section we will investigate a procedure for doing so.

\footnotetext{
${ }^{3}$ See, e.g., Fig. 7 in Ref. 59 for 'typical' jet shapes as generated by different algorithms.
} 


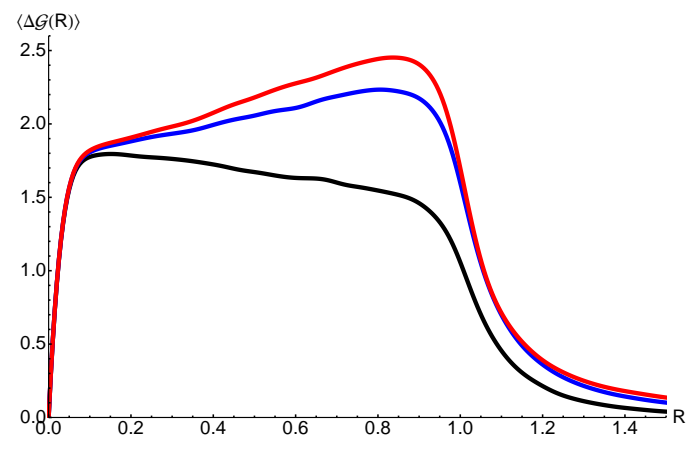

(a) Pythia8

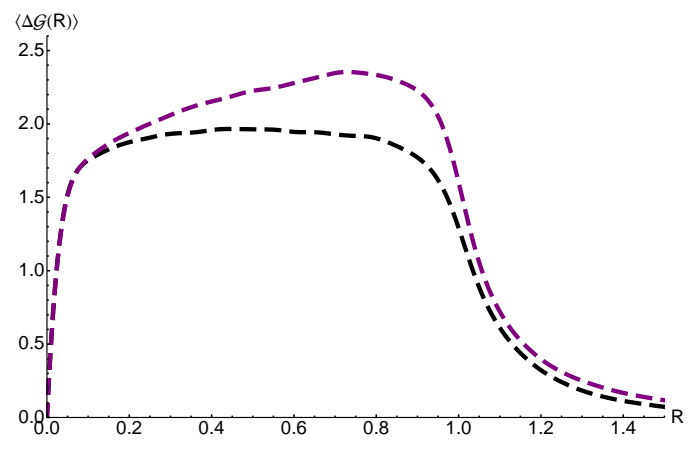

(b) Herwig ++

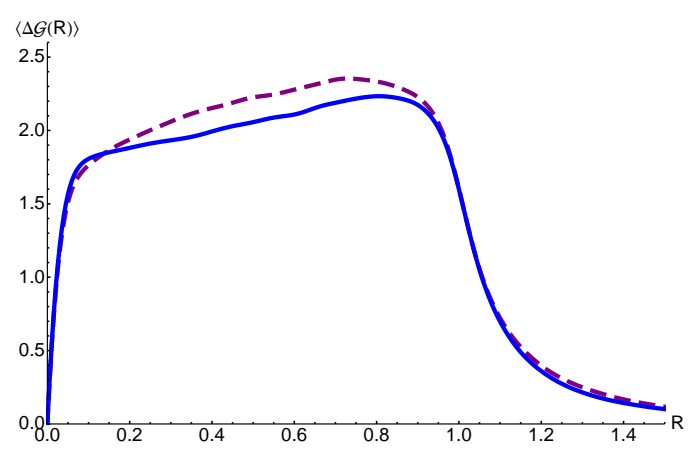

(c) Pythia8 vs. Herwig ++

Figure 4.4: Average angular structure functions for ensembles of jets with $p_{T}>200 \mathrm{GeV}$. The black curves have no underlying event nor any ISR. In contrast to the previous section, here underlying event and ISR are turned on for the colored curves. On the LHS, the Pythia8 samples make use of tune $4 \mathrm{C}$, with the red curve having twice as much UE activity as the blue curve. On the RHS, the purple curve corresponds to Herwig++ tune LHC-UE72. For comparison, the bottom figure overlays the Pythia8 and Herwig ++ curves. These are anti-kT jets with jet radius $R_{0}=1.0$. See Appendix C for more details about the Monte Carlo. 


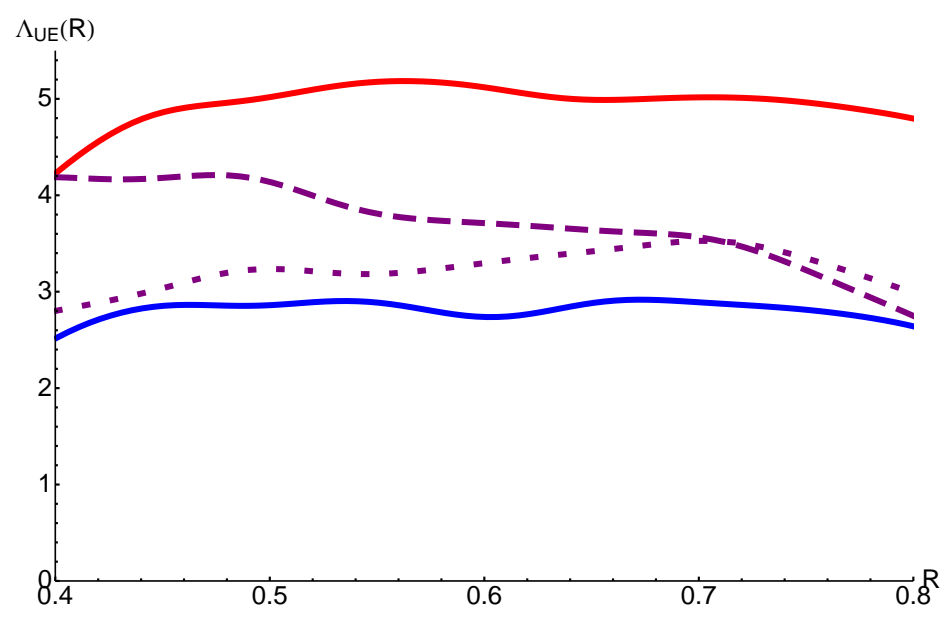

Figure 4.5: $\Lambda_{\mathrm{UE}}(R)$ curves as extracted via the procedure detailed in Sec. 4.4.2. The solid curves correspond to the Pythia8 samples in Fig. 4.4, while the dashed curve corresponds to the Herwig ++ sample. The dotted curve corresponds to the same Herwig ++ sample as the dashed curve, the difference being that the power law of the underlying event ansatz is changed from $R^{4}$ to $R^{3.3}$. The matching was done at $R_{\min }=0.25$.

\subsubsection{Procedure}

One possible procedure for extracting $\Lambda_{\mathrm{UE}}$ for a given ensemble of jets works from the assumption that $\langle\Delta \mathcal{G}(R)\rangle_{\text {pert }}$ is approximately flat in $R$. We have seen some tentative evidence for this hypothesis in Sec. 4.3. We can estimate $\langle\Delta \mathcal{G}(R)\rangle_{\text {pert }}$ by measuring $\langle\Delta \mathcal{G}(R)\rangle$ at small $R$. Specifically we assume that the perturbative piece is exactly flat and given as

$$
\langle\Delta \mathcal{G}(R)\rangle_{\text {pert }}=\left\langle\Delta \mathcal{G}\left(R_{\min }\right)\right\rangle \equiv C
$$

where $R_{\min } \ll 1$. We choose $R_{\min }=0.25$. With this ansatz, we can invert Eq. 4.23 to solve for $\Lambda_{\mathrm{UE}}$ as a function of $R$ :

$$
\Lambda_{\mathrm{UE}}(R)=\frac{2\langle\mathcal{G}(R)\rangle}{\pi p_{T} R^{4}} \frac{\langle\Delta \mathcal{G}(R)\rangle-C}{4-C}
$$

The flatness of $\Lambda_{\mathrm{UE}}(R)$ over a wide range of $R$ can then justify the ansatz a posteriori, and $\Lambda_{\mathrm{UE}}(R)$ can be averaged over a range in $R$ to obtain $\bar{\Lambda}_{\mathrm{UE}}$. Summarizing, the procedure would be: 
1. Measure $C \equiv\left\langle\Delta \mathcal{G}\left(R_{\min }\right)\right\rangle$ and take this as an ansatz for the perturbative contribution to $\langle\Delta \mathcal{G}(R)\rangle$ for all $R$.

2. Construct Eq. 4.25 for a range of $R$ with $R>R_{\min }$.

3. Then $\bar{\Lambda}_{\mathrm{UE}}$, the average value of $\Lambda_{\mathrm{UE}}(R)$ over a range in $R$, gives a measure of the average density of UE in the ensemble of jets.

An additional sanity check on the extracted scales could be to measure $\bar{\Lambda}_{\mathrm{UE}}$ as a function of the number of primary vertices $n_{\mathrm{PV}}$. If the above procedure makes sense, $\bar{\Lambda}_{\mathrm{UE}}$ should exhibit a clear linear dependence on $n_{\mathrm{PV}}$, since pile-up will contribute uncorrelated radiation to the ensemble of jets in an amount proportional to $n_{\mathrm{PV}}-1$.

\subsubsection{Results}

Curves $\Lambda_{\mathrm{UE}}(R)$ as extracted from Monte Carlo data via this procedure are plotted in Fig. 4.5. For Pythia8, $\Lambda_{\mathrm{UE}}(R)$ is approximately flat over a broad range in $R$, justifying the ansatz in Eq. 4.22. Note that the scale of the red curve is about twice that of the blue curve. This is as expected, since the red sample has twice as much UE activity as the blue sample (but the same amount of ISR). The dashed Herwig++ curve, by contrast, is not flat and has an unambiguous downward slope. Changing the UE power law ansatz from $R^{4}$ to $R^{3.3}$ results in a $\Lambda_{\mathrm{UE}}(R)$ curve that is much flatter, though still at a similar scale. That is to say that for Herwig ++ the underlying event is not uniformly distributed, instead being clustered somewhat around the center of the hard, perturbative jet. This points to the possibility of fitting for the exponent in the UE ansatz of Eq. 4.22, but we do not explore this possibility any further.

The average UE densities $\bar{\Lambda}_{\mathrm{UE}}$ defined from the extracted curves $\Lambda_{\mathrm{UE}}(R)$ are listed in Table 4.1. These are defined by averaging $\Lambda_{\mathrm{UE}}(R)$ in the range from $R=0.4$ to $R=0.8$, with the perturbative contribution to $\langle\Delta \mathcal{G}(R)\rangle$ matched at $R_{\min }=0.25$. Also listed are the average transverse momentum densities $\Lambda_{\text {Trans }}$ in the transverse region (for the definition of which see Sec. 4.5). For all the samples we see that $\bar{\Lambda}_{\mathrm{UE}}$ tracks $\Lambda_{\text {Trans }}$ as expected, although it tends to be somewhat larger. For the three Pythia8 samples we see how, as we scale the amount of UE activity, $\Lambda_{\text {Trans }}$ and $\bar{\Lambda}_{\mathrm{UE}}$ rise linearly and in tandem. 


\begin{tabular}{l|c|c} 
Monte Carlo Sample & $\Lambda_{\text {Trans }}$ & $\bar{\Lambda}_{\mathrm{UE}}$ \\
\hline \hline Pythia8 Tune 4C & $3.2 \mathrm{GeV}$ & $2.8 \pm 0.3 \mathrm{GeV}$ \\
Pythia8 Tune 4C' & $4.6 \mathrm{GeV}$ & $5.0 \pm 0.7 \mathrm{GeV}$ \\
Pythia8 Tune 4C' & $6.0 \mathrm{GeV}$ & $7.2 \pm 1.1 \mathrm{GeV}$ \\
Herwig++ Tune LHC-UE7-2 & $3.3 \mathrm{GeV}$ & $3.7 \pm 1.0 \mathrm{GeV}$ \\
Herwig++ Tune LHC-UE7-2 $\left(R^{3.3}\right.$ ansatz $)$ & $3.3 \mathrm{GeV}$ & $3.2 \pm 0.4 \mathrm{GeV}$
\end{tabular}

Table 4.1: Table of extracted UE densities. $\Lambda_{\text {Trans }}$ is the average $p_{T}$ density in the transverse region and $\bar{\Lambda}_{\mathrm{UE}}$ is extracted from $\langle\Delta \mathcal{G}(R)\rangle$. The 'error' bars quoted for $\bar{\Lambda}_{\mathrm{UE}}$ are the maximum difference of the function $\Lambda_{\mathrm{UE}}(R)$ from its average, which is computed in the range from $R=0.4$ to $R=0.8$. Tunes $4 \mathrm{C}^{\prime}$ and $4 \mathrm{C}^{\prime \prime}$ differ from tune $4 \mathrm{C}$ in that they have twice and thrice as much UE activity, respectively. See Appendix $\mathrm{C}$ for more details about the Monte Carlo.

We can ask how sensitive the extraction of $\bar{\Lambda}_{\mathrm{UE}}$ is to deviations from the flatness assumption. The largest dependence on the perturbative ansatz $C$ in Eq. 4.25 is through the term $\langle\Delta \mathcal{G}(R)\rangle-C$. A large overestimation (underestimation) of $C$ gives a large underestimation (overestimation) of the average UE density. For example, if for Pythia8 Tune $4 \mathrm{C}$ we let $C$ be given by the $\langle\Delta \mathcal{G}(R)\rangle$ obtained from Pythia8 in the absence of UE/ISR, we find an $\mathcal{O}(100 \%)$ correction to $\bar{\Lambda}_{\mathrm{UE}}$. In contrast the corresponding correction for Herwig ++ is only $\mathcal{O}(20 \%)$.

The validity of the procedure explored in this section depends on a number of assumptions, and an assessment of its usefulness would require a detailed experimental study with a particular emphasis on the systematic uncertainties involved. In addition, a better theoretical understanding of the perturbative contribution to $\langle\Delta \mathcal{G}(R)\rangle$ would be needed to determine the degree to which the flatness assumption is warranted. Nevertheless, as the luminosity (and eventually the center of mass energy) continues to rise at the LHC, the increasing importance of UE and PU motivates the study of additional experimental handles on the impact of UE and PU on hadronic final state reconstruction. While something like the "jet-area/median" method introduced in Ref. [60] is much more ambitious, since it provides a handle on fluctuations in $\mathrm{UE}$ and $\mathrm{PU}$, the procedure proposed here might be a useful complement to this and other methods. 


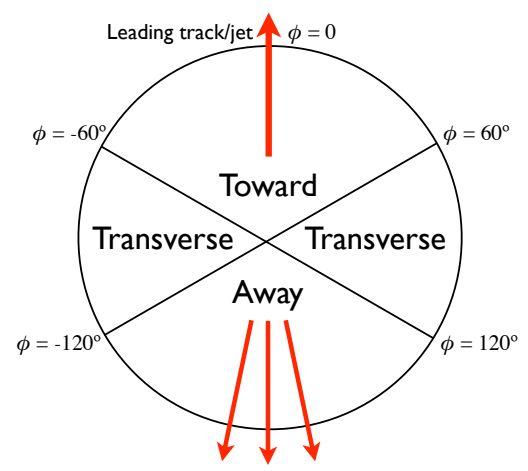

Figure 4.6: Definition of the transverse region.

\subsection{Angular correlations in the transverse region}

To gain more intuition for the scaling information encoded in the average angular structure function, it is interesting to consider a qualitatively different ensemble of jets. Unlike many traditional jet shape observables, $\langle\Delta \mathcal{G}(R)\rangle$ is a meaningful observable for regions of the detector populated with soft, diffuse radiation. In the following we will focus on the transverse region of dijet events, as traditionally employed in underlying event studies. We will explore three different models for the underlying event: (i) the analytically tractable Feynman-Wilson gas; (ii) a toy Monte Carlo that describes uniformly distributed mini-jets; and (iii) full Pythia8 simulation.

The transverse region is defined with respect to the direction of the hardest jet or hardest charged track $(\phi \equiv 0)$, with its two halves extending between $\phi= \pm \pi / 3$ and $\phi= \pm 2 \pi / 3$ and $\eta=-\eta_{\max }$ and $\eta=\eta_{\max }$ (see Fig. 4.6) [61]. We choose to define the transverse region with respect to the hardest jet and set $\eta_{\max }=2.0$. The two transverse regions are further distinguished by their total activity. The region with the largest scalar sum $p_{T}$ is denoted as the trans-max region and the other is the trans-min region. Historically, underlying event studies have often focused on these two regions, since they are less sensitive to contamination from the hard perturbative scattering [62]. In the following ensemble averages will always be defined on charged tracks in the trans-min region, although other variations are possible.

The most commonly used models of the underlying event are based on a picture of hadronic collisions in which multiple parton interactions (MPI) contribute to the 
soft activity in each event [63]. These models proceed by extrapolating the $2 \rightarrow 2$ QCD matrix element to semi-perturbative energies. Spectator partons that are not involved in the hard collision are allowed to scatter, resulting in an approximately uniform population of low $p_{T}$ jets, at least up to moderate pseudorapidities.

\subsubsection{Feynman-Wilson Gas}

Following the MPI hypothesis, we will make a number of simplifying assumptions and see what sort of $\langle\Delta \mathcal{G}(R)\rangle$ we would expect to measure in the trans-min region. To begin with consider a model for the underlying event along the lines of the Feynman-Wilson gas [64]. We assume that the number of underlying event particles is distributed with a probability distribution $\rho(n)$ that depends only on the number of particles $n$. Typically this is taken to be Poissonian. Also, we will assume that the transverse momentum of the underlying event particles has a distribution $p_{T}(n)$ that is uniform in $\eta$ and $\phi$.

In this case the average angular correlation function can be calculated from

$$
\langle\mathcal{G}(R)\rangle=\sum_{n=2}^{\infty} \rho(n) \sum_{i<j \leq n} p_{T}(n) p_{T}(n) \Delta R_{i j}^{2} \Theta_{\mathrm{dR}}\left(R-\Delta R_{i j}\right)
$$

For fixed $n$, the sum over the pairs of constituents breaks up into $\left(\begin{array}{l}n \\ 2\end{array}\right)$ identical terms so that we can write

$$
\langle\mathcal{G}(R)\rangle=\sum_{n=2}^{\infty}\left(\begin{array}{l}
n \\
2
\end{array}\right) \rho(n) p_{T}(n) p_{T}(n) \int[d \eta d \phi] \Delta R_{12}^{2} \Theta_{\mathrm{dR}}\left(R-\Delta R_{12}\right)
$$

where $[d \eta d \phi] \equiv d \eta_{1} d \eta_{2} d \phi_{1} d \phi_{2}$. Since all the $R$ dependence is in the integral, taking the logarithmic derivative yields the simple expression:

$$
\langle\Delta \mathcal{G}(R)\rangle \equiv \frac{d \log \langle\mathcal{G}(R)\rangle}{d \log R}=R \frac{\int[d \eta d \phi] \Delta R_{12}^{2} \delta_{\mathrm{dR}}\left(R-\Delta R_{12}\right)}{\int[d \eta d \phi] \Delta R_{12}^{2} \Theta_{\mathrm{dR}}\left(R-\Delta R_{12}\right)}
$$

That is, assuming that the underlying event is uniformly distributed, we can compute $\langle\Delta \mathcal{G}(R)\rangle$ without knowing the $p_{T}$ or particle number distributions. The average angular structure function becomes purely geometric. 


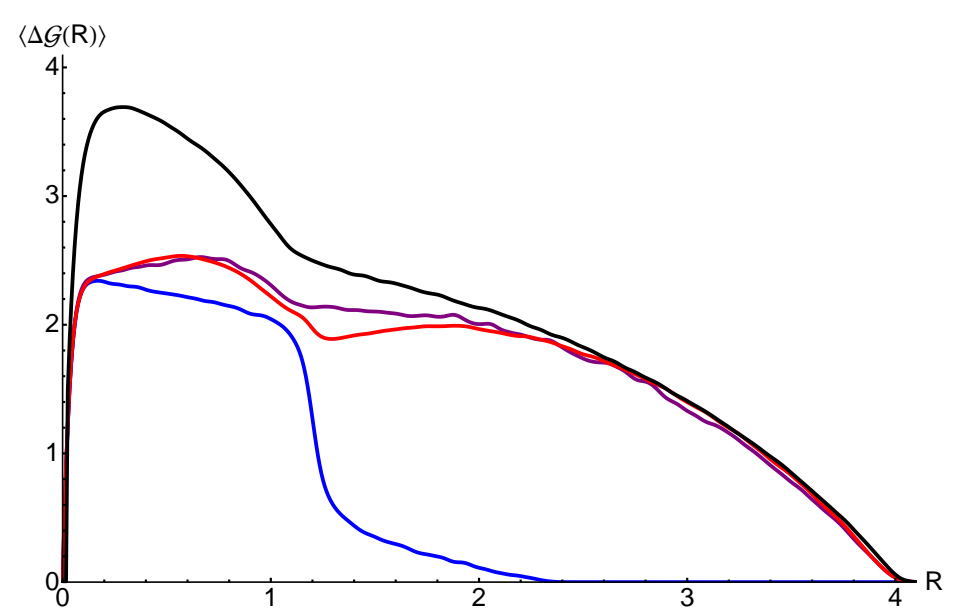

Figure 4.7: $\langle\Delta \mathcal{G}(R)\rangle$ in the trans-min region. The black curve corresponds to the FeynmanWilson gas. The purple curve corresponds to Pythia8 dijet events with $p_{T \text { jet }}>200 \mathrm{GeV}$. The red curve corresponds to the toy Monte Carlo, described below. Finally, the blue curve corresponds to an ensemble of single "DLA mini-jets" as employed in the toy Monte Carlo. See Appendix C for more details about the Monte Carlo.

For a rectangle of size $\Delta \eta \times \Delta \phi,\langle\Delta \mathcal{G}(R)\rangle$ can be computed for $d R=0$. For example, for $R<\min (\Delta \eta, \Delta \phi)$ we have:

$$
\langle\Delta \mathcal{G}(R)\rangle=\frac{2 \pi \Delta \eta \Delta \phi-4(\Delta \eta+\Delta \phi) R+2 R^{2}}{\frac{\pi}{2} \Delta \eta \Delta \phi-\frac{4}{5}(\Delta \eta+\Delta \phi) R+\frac{1}{3} R^{2}}=4-\frac{8}{5 \pi} \frac{\Delta \eta+\Delta \phi}{\Delta \eta \Delta \phi} R+\mathcal{O}\left(R^{2}\right)
$$

The corresponding $\langle\Delta \mathcal{G}(R)\rangle$ over the full range of $R$ with $d R=0.04, \Delta \phi=\pi / 3$, and $\Delta \eta=4.0$ is plotted as the black curve in Fig. 4.7. Edge effects quickly bring $\langle\Delta \mathcal{G}(R)\rangle$ down to zero for $R \gtrsim 1$. At small but nonzero $R,\langle\Delta \mathcal{G}(R)\rangle$ approaches the value of 4 that is obtained in the infinite-plane limit $\Delta \eta, \Delta \phi \rightarrow \infty$. As $R \rightarrow 0$ the smoothing pulls $\langle\Delta \mathcal{G}(R)\rangle$ down from near 4 , as would be the case for $d R=0$ (see Eq. 4.29).

\subsubsection{Toy Monte Carlo}

With Monte Carlo we can explore the $\langle\Delta \mathcal{G}(R)\rangle$ that results from underlying event models more complicated than the simplest Feynman-Wilson gas. In particular we would like to understand the $\langle\Delta \mathcal{G}(R)\rangle$ that results from MPI models. We use the following toy Monte Carlo to test whether we understand the key physics ingredients 


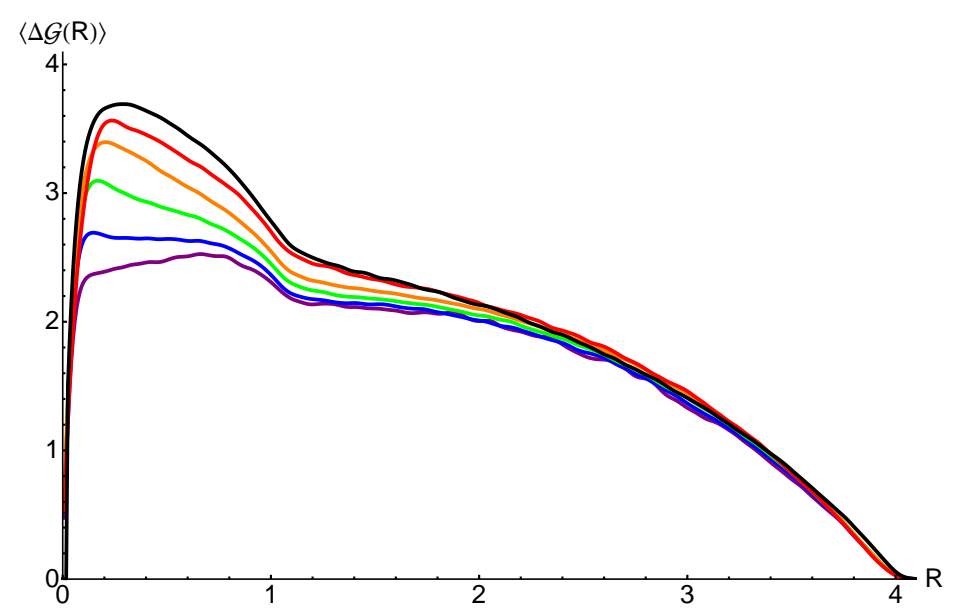

Figure 4.8: $\langle\Delta \mathcal{G}(R)\rangle$ for ensembles excluding events where the $p_{T}$ of the hardest charged particle in the trans-min region exceeds a given $p_{T \max } \cdot\langle\Delta \mathcal{G}(R)\rangle$ for the Feynman-Wilson gas is shown in black, while the colored curves correspond to Pythia8 dijet events with $p_{T \text { jet }}>200 \mathrm{GeV}$. The purple curve has no $p_{T}$ cut, while the blue, green, orange and red curves correspond to $p_{T \max }$ of $16 \mathrm{GeV}, 8 \mathrm{GeV}, 4 \mathrm{GeV}$ and $1 \mathrm{GeV}$, respectively. See Appendix C] for more details about the Monte Carlo.

entering into the form of $\langle\Delta \mathcal{G}(R)\rangle$ obtained from Pythia8. First, we generate $n$ minijets, where $n$ is selected from a Poisson distribution with mean $\langle n\rangle=0.30$ per unit area of azimuth/pseudorapidity. We choose the transverse momenta of the mini-jets from the distribution

$$
\rho\left(p_{T}\right) \sim \frac{d p_{T}^{2}}{p_{T}^{4}} \Theta\left(p_{T}^{2}-\lambda^{2}\right)
$$

where $\lambda=1 \mathrm{GeV}$. To generate the emissions that make up each mini-jet we employ a sort of double logarithmic approximation (DLA) scheme that mimics the parton shower. That is, for each mini-jet we generate a fixed number of emissions, $N_{g}=10$, where each "gluon" is generated according to the distribution

$$
w(\theta, z, \phi) \sim \frac{d \theta}{\theta} \frac{d z}{z} \frac{d \phi}{2 \pi}
$$

where $\theta$ is the angular separation between the gluon and the center of the mini-jet, $z$ is the energy fraction of the gluon, and $\phi$ is the azimuthal angle around the mini-jet axis. The $\theta$ and $z$ distributions are cut off at finite values, with $0.1<z<1.0$ and $0.01<\theta<1.2$. 
The $\langle\Delta \mathcal{G}(R)\rangle$ for an ensemble of single "DLA mini-jets" is plotted as the blue curve in Fig. 4.7. By construction, for $R<\theta_{\max }=1.2$ DLA mini-jets have an average angular structure function near the perturbative value of 2 . Since MPI models are dominated by two scaling behaviors, (i) the perturbative $R^{2}$ scaling of the substructure of the mini-jets at small $R$ and (ii) the Feynman-Wilson scaling at large $R$, we expect our toy model to yield a $\langle\Delta \mathcal{G}(R)\rangle$ between the limiting $\langle\Delta \mathcal{G}(R)\rangle$ curves of (i) and (ii). This is what we see in Fig. 4.7, where the red curve interpolates between the blue and black curves, with a smooth transition between the two regimes at intermediate $R$. Furthermore, we see that the toy Monte Carlo does a good job of describing the average angular structure function obtained from Pythia8.

\subsubsection{Emergence of jets}

In Fig. 4.8 we illustrate the effect on $\langle\Delta \mathcal{G}(R)\rangle$ when the ensemble is defined to exclude events where the $p_{T}$ of the hardest charged particle in the trans-min region exceeds a given $p_{T \max }$. The Feynman-Wilson curve is shown for comparison in black. As the cut is decreased from infinity, $\langle\Delta \mathcal{G}(R)\rangle$ approaches the Feynman-Wilson curve at small $R$. This is because the $p_{T}$ cut has the effect of removing events with harder (and therefore jettier) structure in the transverse region. The soft events that remain are composed of soft mini-jets whose substructure is less jetty due to the smaller dynamical range of the parton shower. This effect should be measurable in data.

\subsection{Discussion and conclusions}

The angular correlation function provides a direct probe into the scaling behavior of QCD. Because it is formulated in terms of two-particle correlations, it asks a particularly detailed question about jet substructure. Many jet shape observables (the integrated jet shape, angularities, etc.) are linear in the momenta of the jet constituents and are explicitly defined with respect to the jet center, i.e. they are radial moments of one kind or another. In this sense they can be thought of as accessing one-particle correlations, and the measurement of $\langle\Delta \mathcal{G}(R)\rangle$ would be expected to provide orthogonal information about the substructure of jets. 
This comment is especially relevant to the observed differences in $\langle\Delta \mathcal{G}(R)\rangle$ between Herwig ++ and Pythia8. If these event generators are being tuned to experimental data for observables like the integrated jet shape, then it is not surprising that they should give different predictions for two-particle correlations. Understanding these differences will be important if measurements of $\langle\Delta \mathcal{G}(R)\rangle$ are to be used for improving Monte Carlo tunes. For example, the evolution variable of the parton shower affects both the structure of emissions in the jet as well as the scale at which the running coupling is evaluated. In addition, numerous other effects such as hadronization, color reconnections, and the details of the UE model will enter into the prediction for $\langle\Delta \mathcal{G}(R)\rangle$. One would also like to understand how matched samples of jets, with their different treatment of hard, wide-angle radiation, affect the form of $\langle\Delta \mathcal{G}(R)\rangle$.

An especially interesting property of $\langle\Delta \mathcal{G}(R)\rangle$ is that it can be interpreted as an average scaling exponent. This makes the leading order result $\langle\Delta \mathcal{G}(R)\rangle=2$ particularly simple to understand and provides useful intuition for the higher order effects explored in Sec. 4.3. It also forms the basis of the two applications of $\langle\Delta \mathcal{G}(R)\rangle$ examined in Sec. 4.4 and Sec. 4.5. In the first case, reliable extraction of $\bar{\Lambda}_{\mathrm{UE}}$ will require a better theoretical understanding of the perturbative contributions to $\langle\Delta \mathcal{G}(R)\rangle$. In particular it remains to determine to what degree the flatness assumption made in Sec. 4.4.2 is warranted. In the second case, forming the ensemble average in the transverse region provides additional insight into the physics encoded in $\langle\Delta \mathcal{G}(R)\rangle$. We find that MPI models predict a quasi-universal form for $\langle\Delta \mathcal{G}(R)\rangle$ with $\langle\Delta \mathcal{G}(R)\rangle \simeq 2$ at small $R$ and the large $R$ form following the Feynman-Wilson gas. Although the jetty nature of UE is already well established, a measurement of $\langle\Delta \mathcal{G}(R)\rangle$ in the transverse region has the nice property that it exhibits both the perturbative substructure and the uniform distribution of mini-jets.

In this paper we have argued that the ensemble average of the angular structure function makes for a particularly interesting jet shape observable. From a theoretical point of view its interpretation as a scaling exponent is especially compelling. From an experimental point of view the possibility of measuring the average contribution of the underlying event and pile-up to hard perturbative jets is intriguing. To go further 
will require experimental input, and we hope that $\langle\Delta \mathcal{G}(R)\rangle$ might be measured at the LHC. 


\section{Appendix A}

\section{Catalogue of massive antenna splitting functions}

In this appendix, we catalogue the various antenna splitting functions for massless particles derived in this paper. Antenna splitting functions not listed are equal to cases listed below that are related by the $P$ and $C$ symmetries of QCD.

\section{A.0.1 Splitting functions with one massive scalar}

A.0.1.1 Spin $\frac{1}{2}$ antenna: initial dipole $S \bar{q}_{L}$

$$
\begin{aligned}
& \mathcal{S}\left(S g_{L} \bar{q}_{L}\right)=\frac{Q}{2 K}\left|\frac{\left\langle A^{b}(b+c) a c\right\rangle}{[c a c\rangle[b c]}\right|^{2} \\
& \mathcal{S}\left(S g_{R} \bar{q}_{L}\right)=\frac{Q}{2 K}\left|\frac{\left\langle A^{b} b\right\rangle\langle b a c]}{[c a c\rangle\langle b c\rangle}\right|^{2}
\end{aligned}
$$

A.0.1.2 Spin 1 antenna: initial dipole $S g_{L}$

$$
\mathcal{S}\left(S g_{L} g_{L}\right)=\frac{1}{(2 K)^{2}}\left|\frac{1}{[b c]}\left[\frac{\left\langle A^{b} b\right\rangle^{2}[b a c\rangle}{[c a c\rangle}+2\left\langle A^{b} c\right\rangle\left\langle A^{b} b\right\rangle+\frac{\left\langle A^{b} c\right\rangle^{2}[c a b\rangle}{[b a b\rangle}\right]\right|^{2}
$$




$$
\begin{aligned}
& \mathcal{S}\left(S g_{R} g_{L}\right)=\frac{1}{(2 K)^{2}}\left|\frac{\left\langle A^{b} b\right\rangle^{2}\langle b a c]}{[c a c\rangle\langle b c\rangle}\right|^{2} \\
& \mathcal{S}\left(S g_{L} g_{R}\right)=\frac{1}{(2 K)^{2}}\left|\frac{\left\langle A^{b} c\right\rangle^{2}\langle c a b]}{[b a b\rangle\langle b c\rangle}\right|^{2} \\
& \mathcal{S}\left(S g_{R} g_{R}\right)=0 \\
& \mathcal{S}\left(S \bar{q}_{R} q_{L}\right)=\frac{1}{(2 K)^{2}} \frac{\left[b A^{b} b\right\rangle^{2}}{[b c b\rangle} \\
& \mathcal{S}\left(S \bar{q}_{L} q_{R}\right)=\frac{1}{(2 K)^{2}} \frac{\left[c A^{b} c\right\rangle^{2}}{[b c b\rangle}
\end{aligned}
$$

Note that the last two expressions are already squared and evaluate to values that are real and positive for the case of a final-state antenna. For example,

$$
\mathcal{S}\left(S \bar{q}_{R} q_{L}\right)=\frac{1}{(2 K)^{2}} \frac{\left(2 b \cdot A^{b}\right)^{2}}{2 b \cdot c} .
$$

\section{A.0.2 Splitting functions with one massive fermion}

A.0.2.1 Spin 0 antenna: initial dipole $Q_{L} \bar{q}_{L}$

$$
\begin{aligned}
& \mathcal{S}\left(Q_{L} g_{L} \bar{q}_{L}\right)=\frac{Q}{2 K}\left|\frac{\left\langle a^{b} c\right\rangle\left[a^{\sharp} Q b\right\rangle}{\left[a^{\sharp} c\right][c a c\rangle}\right|^{2} \\
& \mathcal{S}\left(Q_{L} g_{R} \bar{q}_{L}\right)=\frac{Q}{2 K}\left|\frac{\left\langle a^{b} b\right\rangle[c Q b\rangle}{\langle b c\rangle[c a c\rangle}\right|^{2} \\
& \mathcal{S}\left(Q_{R} g_{L} \bar{q}_{L}\right)=\frac{m^{2} Q}{2 K}\left|\frac{\left\langle a^{\sharp} c\right\rangle\left[a^{b} Q b\right\rangle}{\left\langle a^{\sharp} a^{b}\right\rangle\left[a^{b} c\right][c a c\rangle}\right|^{2} \\
& \mathcal{S}\left(Q_{R} g_{R} \bar{q}_{L}\right)=\frac{m^{2} Q}{2 K}\left|\frac{\left\langle a^{\sharp} b\right\rangle[c Q b\rangle}{\left\langle a^{\sharp} a^{b}\right\rangle\langle b c\rangle[c a c\rangle}\right|^{2}
\end{aligned}
$$

A.0.2.2 Spin $\frac{1}{2}$ antenna: initial dipole $Q_{L} g_{L}$

$$
\mathcal{S}\left(Q_{L} g_{L} g_{L}\right)=\frac{1}{(2 K)^{2}}\left|\frac{\left\langle A^{b} b\right\rangle\left(Q^{2}\left[a^{\sharp} a c\right\rangle-m^{2}\left[a^{\sharp} Q c\right\rangle\right)}{\left[a^{\sharp} a^{b}\right]\langle c a c][b c]}+\frac{\left\langle A^{b} c\right\rangle\left(Q^{2}\left[a^{\sharp} a b\right\rangle-m^{2}\left[a^{\sharp} Q b\right\rangle\right)}{\left[a^{\sharp} a^{b}\right]\langle b a b][b c]}\right|^{2}
$$




$$
\begin{aligned}
& \mathcal{S}\left(Q_{L} g_{R} g_{L}\right)=\frac{1}{(2 K)^{2}}\left|\frac{\left\langle a^{b} b\right\rangle\left\langle A^{b} b\right\rangle\langle b a c]}{\langle c a c]\langle b c\rangle}\right|^{2} \\
& \mathcal{S}\left(Q_{L} g_{L} g_{R}\right)=\frac{1}{(2 K)^{2}}\left|\frac{\left\langle a^{b} c\right\rangle\left\langle A^{b} c\right\rangle\langle c a b]}{\langle b a b]\langle b c\rangle}\right|^{2} \\
& \mathcal{S}\left(Q_{L} g_{R} g_{R}\right)=0 \\
& \mathcal{S}\left(Q_{R} g_{L} g_{L}\right)=\frac{m^{2}}{(2 K)^{2}}\left|\frac{\left\langle A^{b} b\right\rangle\left(\left\langle a^{\sharp} a Q c\right\rangle-Q^{2}\left\langle a^{\sharp} c\right\rangle\right)}{\left\langle a^{\sharp} a^{b}\right\rangle\langle c a c][b c]}+\frac{\left\langle A^{b} c\right\rangle\left(\left\langle a^{\sharp} a Q b\right\rangle-Q^{2}\left\langle a^{\sharp} b\right\rangle\right)}{\left\langle a^{\sharp} a^{b}\right\rangle\langle b a b][b c]}\right|^{2} \\
& \mathcal{S}\left(Q_{R} g_{R} g_{L}\right)=\frac{m^{2}}{(2 K)^{2}}\left|\frac{\left\langle a^{\sharp} b\right\rangle\left\langle A^{b} b\right\rangle\langle b a c]}{\left\langle a^{\sharp} a^{b}\right\rangle\langle c a c]\langle b c\rangle}\right|^{2} \\
& \mathcal{S}\left(Q_{R} g_{L} g_{R}\right)=\frac{m^{2}}{(2 K)^{2}}\left|\frac{\left\langle a^{\sharp} c\right\rangle\left\langle A^{b} c\right\rangle\langle c a b]}{\left\langle a^{\sharp} a^{b}\right\rangle\langle b a b]\langle b c\rangle}\right|^{2} \\
& \mathcal{S}\left(Q_{R} g_{R} g_{R}\right)=0 \\
& \mathcal{S}\left(Q_{L} \bar{q}_{R} q_{L}\right)=\frac{1}{(2 K)^{2}} \frac{\left[b a^{b} b\right\rangle\left[b A^{b} b\right\rangle}{[b c b\rangle} \\
& \mathcal{S}\left(Q_{L} \bar{q}_{L} q_{R}\right)=\frac{1}{(2 K)^{2}} \frac{\left[c a^{b} c\right\rangle\left[c A^{b} c\right\rangle}{[b c b\rangle} \\
& \mathcal{S}\left(Q_{R} \bar{q}_{R} q_{L}\right)=\frac{m^{2}}{(2 K)^{2}} \frac{\left[b a^{\sharp} b\right\rangle\left[b A^{b} b\right\rangle}{\left[a^{\sharp} a a^{\sharp}\right\rangle[b c b\rangle} \\
& \mathcal{S}\left(Q_{R} \bar{q}_{L} q_{R}\right)=\frac{m^{2}}{(2 K)^{2}} \frac{\left[c a^{\sharp} c\right\rangle\left[c A^{b} c\right\rangle}{\left[a^{\sharp} a a^{\sharp}\right\rangle[b c b\rangle}
\end{aligned}
$$

As in A.1.2, the last four expressions here are already squared and evaluate to real, positive values.

A.0.2.3 Spin 1 antenna: initial dipole $Q_{L} \bar{q}_{R}$

$$
\begin{aligned}
& \mathcal{S}\left(Q_{L} g_{R} \bar{q}_{R}\right)=\frac{1}{(2 K)^{2}}\left|\frac{\left\langle a^{b} B\right\rangle\left[A^{b}(b+c) a c\right]}{\langle c a c]\langle b c\rangle}\right|^{2} \\
& \mathcal{S}\left(Q_{L} g_{L} \bar{q}_{R}\right)=\frac{1}{(2 K)^{2}}\left|\frac{\left[A^{b} b\right]\left(\left[a^{\sharp} a c\right\rangle[b Q B\rangle+m^{2}\langle c B\rangle\left[a^{\sharp} b\right]\right)}{\left[a^{\sharp} a^{b}\right]\langle c a c][b c]}\right|^{2} \\
& \mathcal{S}\left(Q_{R} g_{R} \bar{q}_{R}\right)=\frac{m^{2}}{(2 K)^{2}}\left|\frac{\left\langle a^{\sharp} B\right\rangle\left[A^{b}(b+c) a c\right]}{\left\langle a^{\sharp} a^{b}\right\rangle\langle c a c]\langle b c\rangle}\right|^{2} \\
& \mathcal{S}\left(Q_{R} g_{L} \bar{q}_{R}\right)=\frac{m^{2}}{(2 K)^{2}}\left|\frac{\left[A^{b} b\right]\left(\left\langle a^{\sharp} B\right\rangle\langle c a b]+\left\langle a^{\sharp} c\right\rangle\langle B c b]\right)}{\left\langle a^{\sharp} a^{b}\right\rangle\langle c a c]\langle b c\rangle}\right|^{2}
\end{aligned}
$$


A.0.2.4 Spin $\frac{3}{2}$ antenna: initial dipole $Q_{R} g_{L}$

$$
\begin{aligned}
& \mathcal{S}\left(Q_{L} g_{L} g_{L}\right)=\frac{m^{2} Q^{2}}{(2 Q K)^{3}}\left|\frac{\left[a^{\sharp} B\right]}{\left[a^{\sharp} a^{b}\right][b c]}\left\{\frac{\left\langle A^{b} b\right\rangle\left\langle A^{b}(b+c) a c\right\rangle}{\langle c a c]}+\frac{\left\langle A^{b} c\right\rangle\left\langle A^{b}(b+c) a b\right\rangle}{\langle b a b]}\right\}\right|^{2} \\
& \mathcal{S}\left(Q_{L} g_{R} g_{L}\right)=\frac{m^{2} Q^{2}}{(2 Q K)^{3}}\left|\frac{\left\langle A^{b} b\right\rangle^{2}\left(\left[a^{\sharp} B\right]\langle b a c]+\left[a^{\sharp} c\right]\langle b c B]\right)}{\left[a^{\sharp} a^{b}\right]\langle c a c]\langle b c\rangle}\right|^{2} \\
& \mathcal{S}\left(Q_{L} g_{L} g_{R}\right)=\frac{m^{2} Q^{2}}{(2 Q K)^{3}}\left|\frac{\left\langle A^{b} c\right\rangle^{2}\left(\left[a^{\sharp} B\right]\langle c a b]+\left[a^{\sharp} b\right]\langle c b B]\right)}{\left[a^{\sharp} a^{b}\right]\langle b a b]\langle b c\rangle}\right|^{2} \\
& \mathcal{S}\left(Q_{L} g_{R} g_{R}\right)=0 \\
& \mathcal{S}\left(Q_{R} g_{L} g_{L}\right)=\frac{Q^{2}}{(2 Q K)^{3}}\left|\frac{\left[a^{b} B\right]}{[b c]}\left\{\frac{\left\langle A^{b} b\right\rangle\left\langle A^{b}(b+c) a c\right\rangle}{\langle c a c]}+\frac{\left\langle A^{b} c\right\rangle\left\langle A^{b}(b+c) a b\right\rangle}{\langle b a b]}\right\}\right|^{2} \\
& \mathcal{S}\left(Q_{R} g_{R} g_{L}\right)=\frac{Q^{2}}{(2 Q K)^{3}}\left|\frac{\left\langle A^{b} b\right\rangle^{2}}{\langle c a c]\langle b c\rangle}\left\{\left[a^{b} c\right]\langle b Q B]+m^{2} \frac{\left\langle a^{\sharp} b\right\rangle}{\left\langle a^{\sharp} a^{b}\right\rangle}[c B]\right\}\right|^{2} \\
& \mathcal{S}\left(Q_{R} g_{L} g_{R}\right)=\frac{Q^{2}}{(2 Q K)^{3}}\left|\frac{\left\langle A^{b} c\right\rangle^{2}}{\langle b a b]\langle b c\rangle}\left\{\left[a^{b} b\right]\langle c Q B]+m^{2} \frac{\left\langle a^{\sharp} c\right\rangle}{\left\langle a^{\sharp} a^{b}\right\rangle}[b B]\right\}\right|^{2} \\
& \mathcal{S}\left(Q_{R} g_{R} g_{R}\right)=0 \\
& \mathcal{S}\left(Q_{L} \bar{q}_{R} q_{L}\right)=\frac{m^{2} Q^{2}}{(2 Q K)^{3}} \frac{\left[B a^{\sharp} B\right\rangle\left[b A^{b} b\right\rangle^{2}}{\left[a^{\sharp} a a^{\sharp}\right\rangle[b c b\rangle} \\
& \mathcal{S}\left(Q_{L} \bar{q}_{L} q_{R}\right)=\frac{m^{2} Q^{2}}{(2 Q K)^{3}} \frac{\left[B a^{\sharp} B\right\rangle\left[c A^{b} c\right\rangle^{2}}{\left[a^{\sharp} a a^{\sharp}\right\rangle[b c b\rangle} \\
& \mathcal{S}\left(Q_{R} \bar{q}_{R} q_{L}\right)=\frac{Q^{2}}{(2 Q K)^{3}} \frac{\left[B a^{b} B\right\rangle\left[b A^{b} b\right\rangle^{2}}{[b c b\rangle} \\
& \mathcal{S}\left(Q_{R} \bar{q}_{L} q_{R}\right)=\frac{Q^{2}}{(2 Q K)^{3}} \frac{\left[B a^{b} B\right\rangle\left[c A^{b} c\right\rangle^{2}}{[b c b\rangle}
\end{aligned}
$$

As in A.1.2, the last four expressions here are already squared and evaluate to real, positive values. 


\section{A.0.3 Splitting functions with two massive scalars}

A.0.3.1 Spin 0 antenna: initial dipole $S_{1} \bar{S}_{2}$

$$
\begin{aligned}
& \mathcal{S}\left(S_{1} g_{L} \bar{S}_{2}\right)=Q^{2}\left|\frac{\langle c a b c\rangle}{\langle c a c][c b c\rangle}\right|^{2} \\
& \mathcal{S}\left(S_{1} g_{R} \bar{S}_{2}\right)=Q^{2}\left|\frac{[c a b c]}{[c a c\rangle\langle c b c]}\right|^{2}
\end{aligned}
$$

\section{A.0.4 Splitting functions with a massive fermion and a massive scalar}

A.0.4.1 Spin $\frac{1}{2}$ antenna: initial dipole $Q_{1} \bar{S}_{2}$

$$
\begin{aligned}
& \mathcal{S}\left(Q_{1 L} g_{L} \bar{S}_{2}\right)=\frac{Q^{2}}{\left(E_{1}+K\right)\left(E_{2}+K\right)}\left|\frac{\left\langle a^{b} B^{b}\right\rangle\langle c a b c\rangle}{\langle c a c][c b c\rangle}-\frac{\left\langle a^{b} c\right\rangle\left\langle c B^{b}\right\rangle}{\langle c a c]}\right|^{2} \\
& \mathcal{S}\left(Q_{1 R} g_{L} \bar{S}_{2}\right)=\frac{m_{1}^{2} Q^{2}}{\left(E_{1}+K\right)\left(E_{2}+K\right)}\left|\frac{1}{\left\langle a^{\sharp} a^{b}\right\rangle}\left\{\frac{\left\langle a^{\sharp} B^{b}\right\rangle\langle c a b c\rangle}{\langle c a c][c b c\rangle}-\frac{\left\langle a^{\sharp} c\right\rangle\left\langle c B^{b}\right\rangle}{\langle c a c]}\right\}\right|^{2} \\
& \mathcal{S}\left(Q_{1 L} g_{R} \bar{S}_{2}\right)=\frac{\left\langle a^{2} B^{b}\right\rangle[c a b c]}{\left(E_{1}+K\right)\left(E_{2}+K\right)}\left|\frac{m_{1}^{2} Q^{2}}{[c a c\rangle\langle c b c]}\right|^{2} \\
& \mathcal{S}\left(Q_{1 L} g_{R} \bar{S}_{2}\right)=\frac{1}{\left(E_{1}+K\right)\left(E_{2}+K\right)}\left|\frac{\left\langle a^{\sharp} B^{b}\right\rangle[c a b c]}{\left\langle a^{\sharp} a^{b}\right\rangle}\right|^{2}
\end{aligned}
$$

\section{A.0.5 Splitting functions with two massive fermions}

A.0.5.1 Spin 0 antenna: initial dipole $Q_{1 L} \bar{Q}_{2 L}$

$$
\begin{aligned}
\mathcal{S}\left(Q_{1 L} g_{L} \bar{Q}_{2 L}\right)=\frac{Q^{2}}{\left(\left(E_{1}+K\right)\left(E_{2}+K\right)\right)^{2}} \mid \frac{\left\langle a^{b} B^{b}\right\rangle\langle c a b c\rangle\left\langle A^{b} b^{b}\right\rangle}{\langle c a c][c b c\rangle} & -\frac{\left\langle a^{b} c\right\rangle\left\langle c B^{b}\right\rangle\left\langle A^{b} b^{b}\right\rangle}{\langle c a c]}-\left.\frac{\left\langle a^{b} B^{b}\right\rangle\left\langle A^{b} c\right\rangle\left\langle c b^{b}\right\rangle}{[c b c\rangle}\right|^{2} \\
\mathcal{S}\left(Q_{1 L} g_{L} \bar{Q}_{2 R}\right)= & \frac{m_{2}^{2} Q^{2}}{\left(\left(E_{1}+K\right)\left(E_{2}+K\right)\right)^{2}} \mid \frac{1}{\left\langle b^{b} b^{\sharp}\right\rangle}\left\{\frac{\left\langle a^{b} B\right\rangle^{b}\langle c a b c\rangle\left\langle A^{b} b^{\sharp}\right\rangle}{\langle c a c][c b c\rangle}\right.
\end{aligned}
$$




$$
\begin{aligned}
& \left.-\frac{\left\langle a^{b} c\right\rangle\left\langle c B^{b}\right\rangle\left\langle A^{b} b^{\sharp}\right\rangle}{\langle c a c]}-\frac{\left\langle a^{b} B^{b}\right\rangle\left\langle A^{b} c\right\rangle\left\langle c b^{\sharp}\right\rangle}{[c b c\rangle}\right\}\left.\right|^{2} \\
& \mathcal{S}\left(Q_{1 R} g_{L} \bar{Q}_{2 L}\right)=\frac{m_{1}^{2} Q^{2}}{\left(\left(E_{1}+K\right)\left(E_{2}+K\right)\right)^{2}} \mid \frac{1}{\left\langle a^{\sharp} a^{b}\right\rangle}\left\{\frac{\left\langle a^{\sharp} B\right\rangle^{b}\langle c a b c\rangle\left\langle A^{b} b^{b}\right\rangle}{\langle c a c][c b c\rangle}\right. \\
& \left.-\frac{\left\langle a^{\sharp} c\right\rangle\left\langle c B^{b}\right\rangle\left\langle A^{b} b^{b}\right\rangle}{\langle c a c]}-\frac{\left\langle a^{\sharp} B^{b}\right\rangle\left\langle A^{b} c\right\rangle\left\langle c b^{b}\right\rangle}{[c b c\rangle}\right\}\left.\right|^{2} \\
& \mathcal{S}\left(Q_{1 R} g_{L} \bar{Q}_{2 R}\right)=\frac{m_{1}^{2} m_{2}^{2} Q^{2}}{\left(\left(E_{1}+K\right)\left(E_{2}+K\right)\right)^{2}} \mid \frac{1}{\left\langle a^{\sharp} a^{b}\right\rangle\left\langle b^{\sharp} b^{b}\right\rangle}\left\{\frac{\left\langle a^{\sharp} B^{b}\right\rangle\langle c a b c\rangle\left\langle A^{b} b^{\sharp}\right\rangle}{\langle c a c][c b c\rangle}\right. \\
& \left.-\frac{\left\langle a^{\sharp} c\right\rangle\left\langle c B^{b}\right\rangle\left\langle A^{b} b^{\sharp}\right\rangle}{\langle c a c]}-\frac{\left\langle a^{\sharp} B^{b}\right\rangle\left\langle A^{b} c\right\rangle\left\langle c b^{\sharp}\right\rangle}{[c b c\rangle}\right\}\left.\right|^{2} \\
& \left.\mathcal{S}\left(Q_{1 L} g_{R} \bar{Q}_{2 L}\right)=\frac{Q^{2}}{\left(\left(E_{1}+K\right)\left(E_{2}+K\right)\right)^{2}} \mid \frac{\left\langle a^{b} B^{b}\right\rangle[c a b c]\left\langle A^{b} b^{b}\right\rangle}{\langle c a c][c b c\rangle}\right\}\left.\right|^{2} \\
& \mathcal{S}\left(Q_{1 L} g_{R} \bar{Q}_{2 R}\right)=\frac{m_{2}^{2} Q^{2}}{\left(\left(E_{1}+K\right)\left(E_{2}+K\right)\right)^{2}}\left|\frac{1}{\left\langle b^{b} b^{\sharp}\right\rangle} \frac{\left\langle a^{b} B^{b}\right\rangle[c a b c]\left\langle A^{b} b^{\sharp}\right\rangle}{\langle c a c][c b c\rangle}\right|^{2} \\
& \mathcal{S}\left(Q_{1 R} g_{R} \bar{Q}_{2 L}\right)=\frac{m_{1}^{2} Q^{2}}{\left(\left(E_{1}+K\right)\left(E_{2}+K\right)\right)^{2}}\left|\frac{1}{\left\langle a^{\sharp} a^{b}\right\rangle} \frac{\left\langle a^{\sharp} B\right\rangle^{b}[c a b c]\left\langle A^{b} b^{b}\right\rangle}{\langle c a c][c b c\rangle}\right|^{2} \\
& \mathcal{S}\left(Q_{1 R} g_{R} \bar{Q}_{2 R}\right)=\frac{m_{1}^{2} m_{2}^{2} Q^{2}}{\left(\left(E_{1}+K\right)\left(E_{2}+K\right)\right)^{2}}\left|\frac{1}{\left\langle a^{\sharp} a^{b}\right\rangle\left\langle b^{\sharp} b^{b}\right\rangle} \frac{\left\langle a^{\sharp} B^{b}\right\rangle[c a b c]\left\langle A^{b} b^{\sharp}\right\rangle}{\langle c a c][c b c\rangle}\right|^{2}
\end{aligned}
$$

\section{A.0.5.2 Spin 1 antenna: initial dipole $Q_{1 L} \bar{Q}_{2 R}$}

$$
\begin{aligned}
& \mathcal{S}\left(Q_{1 L} g_{L} \bar{Q}_{2 L}\right)=\frac{m_{2}^{2} Q^{2}}{\left(\left(E_{1}+K\right)\left(E_{2}+K\right)\right)^{2}}\left|\frac{1}{\left\langle b^{b} b^{\sharp}\right\rangle}\left\{\frac{\left\langle a^{b} B^{b}\right\rangle\langle c a b c\rangle\left[A^{b} b^{\sharp}\right]}{\langle c a c][c b c\rangle}-\frac{\left\langle a^{b} c\right\rangle\left\langle c B^{b}\right\rangle\left[A^{b} b^{\sharp}\right]}{\langle c a c]}\right\}\right|^{2} \\
& \left.\mathcal{S}\left(Q_{1 L} g_{L} \bar{Q}_{2 R}\right)=\frac{Q^{2}}{\left(\left(E_{1}+K\right)\left(E_{2}+K\right)\right)^{2}} \mid \frac{\left\langle a^{b} B^{b}\right\rangle\langle c a b c\rangle\left[A^{b} b^{b}\right]}{\langle c a c][c b c\rangle}-\frac{\left\langle a^{b} c\right\rangle\left\langle c B^{b}\right\rangle\left[A^{b} b^{b}\right]}{\langle c a c]}\right\}\left.\right|^{2} \\
& \mathcal{S}\left(Q_{1 R} g_{L} \bar{Q}_{2 L}\right)=\frac{m_{1}^{2} m_{2}^{2} Q^{2}}{\left(\left(E_{1}+K\right)\left(E_{2}+K\right)\right)^{2}} \mid \frac{1}{\left\langle a^{\sharp} a^{b}\right\rangle\left[b^{b} b^{\sharp}\right]}\left\{\frac{\left\langle a^{\sharp} B^{b}\right\rangle\langle c a b c\rangle\left[A^{b} b^{\sharp}\right]}{\langle c a c][c b c\rangle}-\left.\frac{\left\langle a^{\sharp} c\right\rangle\left\langle c B^{b}\right\rangle\left[A^{b} b^{\sharp}\right]}{\langle c a c]}\right|^{2}\right. \\
& \mathcal{S}\left(Q_{1 R} g_{L} \bar{Q}_{2 R}\right)=\frac{m_{1}^{2} Q^{2}}{\left(\left(E_{1}+K\right)\left(E_{2}+K\right)\right)^{2}}\left|\frac{1}{\left\langle a^{\sharp} a^{b}\right\rangle}\left\{\frac{\left\langle a^{\sharp} B^{b}\right\rangle\langle c a b c\rangle\left[A^{b} b^{b}\right]}{\langle c a c][c b c\rangle}-\frac{\left\langle a^{\sharp} c\right\rangle\left\langle c B^{b}\right\rangle\left\langle A^{b} b^{b}\right\rangle}{\langle c a c]}\right\}\right|^{2} \\
& \mathcal{S}\left(Q_{1 L} g_{R} \bar{Q}_{2 L}\right)=\frac{m_{2}^{2} Q^{2}}{\left(\left(E_{1}+K\right)\left(E_{2}+K\right)\right)^{2}} \mid \frac{1}{\left[b^{b} b^{\sharp}\right]}\left\{\frac{\left\langle a^{b} B^{b}\right\rangle[c a b c]\left[A^{b} b^{\sharp}\right]}{\langle c a c][c b c\rangle}-\left.\frac{\left\langle a^{b} B^{b}\right\rangle\left[A^{b} c\right]\left[c b^{\sharp}\right]}{[c b c\rangle}\right|^{2}\right. \\
& \mathcal{S}\left(Q_{1 L} g_{R} \bar{Q}_{2 R}\right)=\frac{Q^{2}}{\left(\left(E_{1}+K\right)\left(E_{2}+K\right)\right)^{2}}\left|\frac{\left\langle a^{b} B^{b}\right\rangle[c a b c]\left[A^{b} b^{b}\right]}{\langle c a c][c b c\rangle}-\frac{\left\langle a^{b} B^{b}\right\rangle\left[A^{b} c\right]\left[c b^{b}\right]}{[c b c\rangle}\right|^{2}
\end{aligned}
$$


$\mathcal{S}\left(Q_{1 R} g_{R} \bar{Q}_{2 L}\right)=\frac{m_{1}^{2} m_{2}^{2} Q^{2}}{\left(\left(E_{1}+K\right)\left(E_{2}+K\right)\right)^{2}}\left|\frac{1}{\left\langle a^{\sharp} a^{b}\right\rangle\left[b^{b} b^{\sharp}\right]}\left\{\frac{\left\langle a^{\sharp} B^{b}\right\rangle[c a b c]\left[A^{b} b^{b}\right]}{\langle c a c][c b c\rangle}-\frac{\left\langle a^{b} B^{b}\right\rangle\left[A^{b} c\right]\left[c b^{\sharp}\right]}{[c b c\rangle}\right\}\right|^{2}$
$\mathcal{S}\left(Q_{1 R} g_{R} \bar{Q}_{2 R}\right)=\frac{m_{1}^{2} Q^{2}}{\left(\left(E_{1}+K\right)\left(E_{2}+K\right)\right)^{2}}\left|\frac{1}{\left\langle a^{\sharp} a^{b}\right\rangle}\left\{\frac{\left\langle a^{\sharp} B^{b}\right\rangle[c a b c]\left[A^{b} b^{b}\right]}{\langle c a c][c b c\rangle}-\frac{\left\langle a^{b} B^{b}\right\rangle\left[A^{b} c\right]\left[c b^{b}\right]}{[c b c\rangle}\right\}\right|^{2}(\mathrm{~A} .10)$

\section{A.0.6 Splitting functions with pair production of scalars}

A.0.6.1 Spin 0 antenna: initial dipole $g_{L} g_{L}$

$$
\mathcal{S}\left(g_{L} \bar{S} S\right)=\frac{1}{Q^{2}}\left|\frac{\langle a[b, c] a\rangle}{s_{b c}}\right|^{2}
$$

A.0.6.2 Spin $\frac{1}{2}$ antenna: initial dipole $q_{L} g_{L}$

$$
\mathcal{S}\left(q_{L} \bar{S} S\right)=\frac{1}{Q^{2}}\left|\frac{\langle a[b, c] A\rangle}{s_{b c}}\right|^{2}
$$

A.0.6.3 Spin $\frac{3}{2}$ antenna: initial dipole $q_{R} g_{L}$

$$
\mathcal{S}\left(q_{R} \bar{S} S\right)=\frac{1}{Q^{4}}\left|\frac{[a B]\langle A[b, c] A\rangle}{s_{b c}}\right|^{2}
$$

A.0.6.4 Spin 2 antenna: initial dipole $g_{R} g_{L}$

$$
\mathcal{S}\left(g_{R} \bar{S} S\right)=\frac{1}{Q^{6}}\left|\frac{[a B]^{2}\langle A[b, c] A\rangle}{s_{b c}}\right|^{2}
$$

\section{A.0.7 Splitting functions with pair production of fermions}

A.0.7.1 Spin 0 antenna: initial dipole $g_{L} g_{L}$

$$
\begin{aligned}
& \mathcal{S}\left(g_{L} \bar{Q}_{L} Q_{L}\right)=\frac{m^{2}}{Q^{2} s_{b c}^{2}}\left|\frac{\left\langle a Q b^{\sharp}\right]\left\langle c^{b} a\right\rangle}{\left[b^{b} b^{\sharp}\right]}+\frac{\left\langle a Q c^{\sharp}\right]\left\langle b^{b} a\right\rangle}{\left[c^{b} c^{\sharp}\right]}\right|^{2} \\
& \mathcal{S}\left(g_{L} \bar{Q}_{L} Q_{R}\right)=\frac{1}{Q^{2} s_{b c}^{2}}\left|\left\langle a Q c^{b}\right]\left\langle b^{b} a\right\rangle+\frac{m^{2}}{\left[b^{b} b^{\sharp}\right]\left\langle c^{b} c^{\sharp}\right\rangle}\left\langle a Q b^{\sharp}\right]\left\langle c^{\sharp} a\right\rangle\right|^{2} \\
& \mathcal{S}\left(g_{L} \bar{Q}_{R} Q_{L}\right)=\frac{1}{Q^{2} s_{b c}^{2}}\left|\left\langle a Q b^{b}\right]\left\langle c^{b} a\right\rangle+\frac{m^{2}}{\left\langle b^{b} b^{\sharp}\right\rangle\left[c^{b} c^{\sharp}\right]}\left\langle a Q c^{\sharp}\right]\left\langle b^{\sharp} a\right\rangle\right|^{2}
\end{aligned}
$$




$$
\mathcal{S}\left(g_{L} \bar{Q}_{R} Q_{R}\right)=\frac{m^{2}}{Q^{2} s_{b c}^{2}}\left|\frac{\left\langle a Q c^{b}\right]\left\langle b^{\sharp} a\right\rangle}{\left[b^{b} b^{\sharp}\right]}+\frac{\left\langle a Q b^{b}\right]\left\langle c^{\sharp} a\right\rangle}{\left[c^{b} c^{\sharp}\right]}\right|^{2}
$$

A.0.7.2 Spin $\frac{1}{2}$ antenna: initial dipole $q_{L} g_{L}$

$$
\begin{aligned}
& \mathcal{S}\left(q_{L} \bar{Q}_{L} Q_{L}\right)= \frac{m^{2}}{4 Q^{2} s_{b c}^{2}} \mid \frac{\left\langle a Q b^{\sharp}\right]\left\langle c^{b} A\right\rangle}{\left[b^{b} b^{\sharp}\right]}+\frac{\left\langle a Q c^{\sharp}\right]\left\langle b^{b} A\right\rangle}{\left[c^{b} c^{\sharp}\right]} \\
&+\frac{\left\langle A(Q-a) b^{\sharp}\right]\left\langle c^{b} a\right\rangle}{\left[b^{b} b^{\sharp}\right]}+\left.\frac{\left\langle A(Q-a) c^{\sharp}\right]\left\langle b^{b} a\right\rangle}{\left[c^{b} c^{\sharp}\right]}\right|^{2} \\
& \mathcal{S}\left(q_{L} \bar{Q}_{L} Q_{R}\right)= \frac{1}{4 Q^{2} s_{b c}^{2}} \mid\left\langle a Q c^{b}\right]\left\langle b^{b} A\right\rangle+\frac{m^{2}}{\left[b^{b} b^{\sharp}\right]\left\langle c^{b} c^{\sharp}\right\rangle}\left\langle a Q b^{\sharp}\right]\left\langle c^{\sharp} A\right\rangle \\
&+\left\langle A(Q-a) c^{b}\right]\left\langle b^{b} a\right\rangle+\left.\frac{m^{2}}{\left[b^{b} b^{\sharp}\right]\left\langle c^{b} c^{\sharp}\right\rangle}\left\langle A(Q-a) b^{\sharp}\right]\left\langle c^{\sharp} a\right\rangle\right|^{2} \\
& \mathcal{S}\left(q_{L} \bar{Q}_{R} Q_{L}\right)= \frac{1}{4 Q^{2} s_{b c}^{2}} \mid\left\langle a Q b^{b}\right]\left\langle c^{b} A\right\rangle+\frac{m^{2}}{\left\langle b^{b} b^{\sharp}\right\rangle\left[c^{b} c^{\sharp}\right]}\left\langle a Q c^{\sharp}\right]\left\langle b^{\sharp} A\right\rangle \\
&+\left\langle A(Q-a) b^{b}\right]\left\langle c^{b} a\right\rangle+\left.\frac{m^{2}}{\left\langle b^{b} b^{\sharp}\right\rangle\left[c^{b} c^{\sharp}\right]}\left\langle A(Q-a) c^{\sharp}\right]\left\langle b^{\sharp} a\right\rangle\right|^{2} \\
& \mathcal{S}\left(q_{L} \bar{Q}_{R} Q_{R}\right)= \frac{m^{2}}{4 Q^{2} s_{b c}^{2}} \mid \frac{\left\langle a Q c^{b}\right]\left\langle b^{\sharp} A\right\rangle}{\left[b^{b} b^{\sharp}\right]}+\frac{\left\langle a Q b^{b}\right]\left\langle c^{\sharp} A\right\rangle}{\left[c^{b} c^{\sharp}\right]} \\
&+\frac{\left\langle A(Q-a) c^{b}\right]\left\langle b^{\sharp} a\right\rangle}{\left[b^{b} b^{\sharp}\right]}+\left.\frac{\left\langle A(Q-a) b^{b}\right]\left\langle c^{\sharp} a\right\rangle}{\left[c^{b} c^{\sharp}\right]}\right|^{2} \quad(\mathrm{~A} .16)
\end{aligned}
$$

A.0.7.3 Spin $\frac{3}{2}$ antenna: initial dipole $q_{R} g_{L}$

$$
\begin{aligned}
& \mathcal{S}\left(q_{R} \bar{Q}_{L} Q_{L}\right)=\frac{m^{2}}{Q^{4} s_{b c}^{2}}\left|[a B]\left\{\frac{\left\langle A(Q-a) b^{\sharp}\right]\left\langle c^{b} A\right\rangle}{\left[b^{b} b^{\sharp}\right]}+\frac{\left\langle A(Q-a) c^{\sharp}\right]\left\langle b^{b} A\right\rangle}{\left[c^{b} c^{\sharp}\right]}\right\}\right|^{2} \\
& \mathcal{S}\left(q_{R} \bar{Q}_{L} Q_{R}\right)=\frac{1}{Q^{4} s_{b c}^{2}}\left|[a B]\left\{\left\langle A(Q-a) c^{b}\right]\left\langle b^{b} A\right\rangle+\frac{m^{2}}{\left[b^{b} b^{\sharp}\right]\left\langle c^{b} c^{\sharp}\right\rangle}\left\langle A(Q-a) b^{\sharp}\right]\left\langle c^{\sharp} A\right\rangle\right\}\right|^{2} \\
& \mathcal{S}\left(q_{R} \bar{Q}_{R} Q_{L}\right)=\frac{1}{Q^{4} s_{b c}^{2}}\left|[a B]\left\{\left\langle A(Q-a) b^{b}\right]\left\langle c^{b} A\right\rangle+\frac{m^{2}}{\left\langle b^{b} b^{\sharp}\right\rangle\left[c^{b} c^{\sharp}\right]}\left\langle A(Q-a) c^{\sharp}\right]\left\langle b^{\sharp} A\right\rangle\right\}\right|^{2}
\end{aligned}
$$




$$
\mathcal{S}\left(q_{R} \bar{Q}_{R} Q_{R}\right)=\frac{m^{2}}{Q^{4} s_{b c}^{2}}\left|[a B]\left\{\frac{\left\langle A(Q-a) c^{b}\right]\left\langle b^{\sharp} A\right\rangle}{\left[b^{b} b^{\sharp}\right]}+\frac{\left\langle A(Q-a) b^{b}\right]\left\langle c^{\sharp} A\right\rangle}{\left[c^{b} c^{\sharp}\right]}\right\}\right|^{2}
$$

A.0.7.4 Spin 2 antenna: initial dipole $g_{R} g_{L}$

$$
\begin{aligned}
& \mathcal{S}\left(g_{R} \bar{Q}_{L} Q_{L}\right)=\frac{m^{2}}{Q^{6} s_{b c}^{2}}\left|[a B]^{2}\left\{\frac{\left\langle A(Q-a) b^{\sharp}\right]\left\langle c^{b} A\right\rangle}{\left[b^{b} b^{\sharp}\right]}+\frac{\left\langle A(Q-a) c^{\sharp}\right]\left\langle b^{b} A\right\rangle}{\left[c^{b} c^{\sharp}\right]}\right\}\right|^{2} \\
& \mathcal{S}\left(g_{R} \bar{Q}_{L} Q_{R}\right)=\frac{1}{Q^{6} s_{b c}^{2}}\left|[a B]^{2}\left\{\left\langle A(Q-a) c^{b}\right]\left\langle b^{b} A\right\rangle+\frac{m^{2}}{\left[b^{b} b^{\sharp}\right]\left\langle c^{b} c^{\sharp}\right\rangle}\left\langle A(Q-a) b^{\sharp}\right]\left\langle c^{\sharp} A\right\rangle\right\}\right|^{2} \\
& \mathcal{S}\left(g_{R} \bar{Q}_{R} Q_{L}\right)=\frac{1}{Q^{6} s_{b c}^{2}}\left|[a B]^{2}\left\{\left\langle A(Q-a) b^{b}\right]\left\langle c^{b} A\right\rangle+\frac{m^{2}}{\left\langle b^{b} b^{\sharp}\right\rangle\left[c^{b} c^{\sharp}\right]}\left\langle A(Q-a) c^{\sharp}\right]\left\langle b^{\sharp} A\right\rangle\right\}\right|^{2} \\
& \mathcal{S}\left(g_{R} \bar{Q}_{R} Q_{R}\right)=\frac{m^{2}}{Q^{6} s_{b c}^{2}}\left|[a B]^{2}\left\{\frac{\left\langle A(Q-a) c^{b}\right]\left\langle b^{\sharp} A\right\rangle}{\left[b^{b} b^{\sharp}\right]}+\frac{\left\langle A(Q-a) b^{b}\right]\left\langle c^{\sharp} A\right\rangle}{\left[c^{b} c^{\sharp}\right]}\right\}\right|^{2} \quad(\mathrm{~A} .18)
\end{aligned}
$$




\section{Appendix B}

\section{Spin-dependent Altarelli-Parisi functions for massive particles}

In this Appendix, we present the spin-dependent Altarelli-Parisi splitting functions for massless and massive particles. The massless cases were derived in the original paper of Altarelli and Parisi [16]. Spin-summed Altarelli-Parisi functions for the cases with massive particles arise in NLO QCD calculations for supersymmetric particle production. They have been catalogued by Catani, Dittmaier, and Trócsányi in [27]. The spin-dependent functions can be worked out by textbook methods. Here we present these functions in a representation convenient for comparison to the antenna splitting functions derived in this paper. We omit the overall color factor of $N_{c}$ and divide by 2 so that the splitting accounts the contents of an individual antenna.

Note that, since we work at the leading order in $N_{c}$ and normalize to a single antenna, there is no difference between the splitting function for a heavy quark or a gluino to radiate a gluon. Thus, there are only two cases, the cases of a heavy scalar $S$ or a heavy quark $Q$ radiating a gluon. The cases of a heavy particle splitting to a heavy particle by radiating a gluon are given by the same expressions with $z \rightarrow(1-z)$.

For $S \rightarrow g S$,

$$
P\left(S \rightarrow S g_{L} S\right)=\frac{p_{T}^{2}}{p_{T}^{2}+z^{2} m^{2}} \frac{1-z}{z}
$$




$$
P\left(S \rightarrow S g_{R} S\right)=\frac{p_{T}^{2}}{p_{T}^{2}+z^{2} m^{2}} \frac{1-z}{z}
$$

For $Q \rightarrow g Q$,

$$
\begin{aligned}
P\left(Q_{L} \rightarrow Q_{L} g_{L}\right) & =\frac{p_{T}^{2}}{p_{T}^{2}+z^{2} m^{2}} \frac{1}{z} \\
P\left(Q_{L} \rightarrow Q_{L} g_{R}\right) & =\frac{p_{T}^{2}}{p_{T}^{2}+z^{2} m^{2}} \frac{(1-z)^{2}}{z} \\
P\left(Q_{L} \rightarrow Q_{R} g_{L}\right) & =\frac{m^{2}}{p_{T}^{2}+z^{2} m^{2}} \frac{z^{4}}{z} \\
P\left(Q_{L} \rightarrow Q_{R} g_{R}\right) & =0
\end{aligned}
$$




\section{Appendix C}

\section{Monte Carlo}

Here we give details of how the various Monte Carlo samples were generated for the study in Chap. 4. Throughout Herwig++ refers to Herwig++ v. 2.5.1 [65-68], and Pythia8 refers to Pythia8 v. 8.150 [14, 70, 71]. To cluster jets, we use the FastJet v. 2.4.2 [72] implementation of the anti-kT algorithm [9]. Note that the final state shower in Herwig ++ is angular-ordered, while Pythia8 has a $p_{T}$-ordered shower. We simulate $p p$ collisions at a center of mass energy of $E_{\mathrm{CM}}=7 \mathrm{TeV}$. No attempt is made to model detector effects, with particle-level information being used in all cases. In Sec. 4.3 , the samples have been generated with MPI and ISR turned off. In Herwig++ this is done via the flags

ShowerHandler:MPIHandler NULL
SplittingGenerator:ISR No

while in Pythia8 it is accomplished via the flags

$$
\begin{aligned}
& \text { PartonLevel:MI = off } \\
& \text { SpaceShower:QCDshower = off }
\end{aligned}
$$

Also, in Sec. 4.3 we use ensembles composed of either quark or gluon jets. These are obtained by choosing the hardest jet in each event from samples generated with pure quark or glue final states, i.e. $g g \rightarrow q \bar{q}$ and $q \bar{q} \rightarrow q \bar{q}$ in the case of quark jets and $g g \rightarrow g g$ and $q \bar{q} \rightarrow g g$ in the case of gluon jets. For the event samples in Sec. 4.4 
MPI and ISR are again turned on. For the underlying event Herwig ++ makes use of tune 'LHC-UE7-2' [69] and Pythia8 makes use of tune '4C' [71, 73]. In all cases we have checked that the $p_{T}$ distributions are similar between corresponding Pythia8 and Herwig++ samples. Also, in Sec. 4.4 we use two Pythia8 event samples (tunes $4 \mathrm{C}^{\prime}$ and $4 \mathrm{C}^{\prime \prime}$ ) in which MPI activity has been increased by factors of 2.0 and 3.0. This is done by dialing the parameter MultipleInteractions:Kfactor.

Finally, in Sec. 4.5 the ensemble averages over the trans-min region come from a dijet sample where the $p_{T}$ of the leading jet is greater than $200 \mathrm{GeV}$. The dijet sample is generated with Pythia8 using tune $4 \mathrm{C}$, and the ensemble averages only make use of charged particles. Because of the large angular separations between charged tracks that can occur in the trans-min region, the ensemble average is modified to include a $R_{\text {min }}$ prescription. That is, a given event only contributes to the numerator and denominator of $\langle\Delta \mathcal{G}(R)\rangle$ in Eq. 4.6 for $R \geq R_{\min }$, where $R_{\min }$ is the minimal angular separation between charged tracks in the trans-min region of that particular event. 


\section{Bibliography}

[1] CERN-LHCC-99-14.

[2] CERN-LHCC-99-15.

[3] G. L. Bayatian et al. [CMS Collaboration], J. Phys. G G 34, 995 (2007).

[4] G. 't Hooft, Nucl. Phys. B 72, 461 (1974).

[5] E. Farhi, Phys. Rev. Lett. 39, 1587 (1977).

[6] G. F. Sterman and S. Weinberg, Phys. Rev. Lett. 39, 1436 (1977).

[7] S. Catani, Y. L. Dokshitzer, M. H. Seymour et al., Nucl. Phys. B406, 187224 (1993); S. D. Ellis, D. E. Soper, Phys. Rev. D48, 3160-3166 (1993). arXiv:hep-ph/9305266.

[8] Y. L. Dokshitzer, G. D. Leder, S. Moretti, B. R. Webber, JHEP 9708, 001 (1997). arXiv:hep-ph/9707323; M. Wobisch, T. Wengler, arXiv:hep-ph/9907280; M. Wobisch, DESY-THESIS-2000-049.

[9] M. Cacciari, G. P. Salam and G. Soyez, JHEP 0804, 063 (2008) [arXiv:0802.1189 [hep-ph]].

[10] A. Abdesselam, E. B. Kuutmann, U. Bitenc, G. Brooijmans, J. Butterworth, P. Bruckman de Renstrom, D. Buarque Franzosi, R. Buckingham et al., [arXiv:1012.5412 [hep-ph]].

[11] A. Altheimer, S. Arora, L. Asquith, G. Brooijmans, J. Butterworth, M. Campanelli, B. Chapleau and A. E. Cholakian et al., arXiv:1201.0008. 
[12] L. G. Almeida, R. Alon and M. Spannowsky, arXiv:1110.3684].

[13] M. Jankowiak, A. J. Larkoski, JHEP 1106, 057 (2011). arXiv:1104.1646.

[14] T. Sjostrand, S. Mrenna and P. Skands, JHEP 0605, 026 (2006) [arXiv:hep$\mathrm{ph} / 0603175]$.

[15] G. Corcella et al., JHEP 0101, 010 (2001) [arXiv:hep-ph/0011363], arXiv:hep$\mathrm{ph} / 0210213$.

[16] G. Altarelli and G. Parisi, Nucl. Phys. B 126, 298 (1977).

[17] Y. L. Dokshitzer, Sov. Phys. JETP 46, 641 (1977) [Zh. Eksp. Teor. Fiz. 73, 1216 (1977)].

[18] G. Marchesini and B. R. Webber, Nucl. Phys. B 238, 1 (1984).

[19] G. Gustafson and U. Pettersson, Nucl. Phys. B 306, 746 (1988); B. Andersson, G. Gustafson and L. Lonnblad, Nucl. Phys. B 339, 393 (1990).

[20] U. Pettersson, Lund preprint LU-TP-88-5 (1988); L. Lonnblad, Comput. Phys. Commun. 71, 15 (1992).

[21] W. T. Giele, D. A. Kosower and P. Z. Skands, Phys. Rev. D 78, 014026 (2008) [arXiv:0707.3652 [hep-ph]].

[22] J. C. Winter and F. Krauss, JHEP 0807, 040 (2008) [arXiv:0712.3913 [hep-ph]].

[23] M. E. Peskin, in preparation.

[24] S. Catani and M. H. Seymour, Phys. Lett. B 378, 287 (1996), [arXiv:hepph/9602277]. Nucl. Phys. B 485, 291 (1997) [Erratum-ibid. B 510, 503 (1998)] [arXiv:hep-ph/9605323].

[25] D. A. Kosower, Phys. Rev. D 57, 5410 (1998) [arXiv:hep-ph/9710213].

[26] J. M. Campbell and E. W. N. Glover, Nucl. Phys. B 527, 264 (1998) [arXiv:hep$\mathrm{ph} / 9710255]$. 
[27] S. Catani and M. Grazzini, Phys. Lett. B 446, 143 (1999) [arXiv:hepph/9810389].

[28] C. Duhr and F. Maltoni, JHEP 0811, 002 (2008) [arXiv:0808.3319 [hep-ph]].

[29] A. Gehrmann-De Ridder, T. Gehrmann and E. W. N. Glover, Phys. Lett. B 612, 49 (2005) [arXiv:hep-ph/0502110],

[30] A. Gehrmann-De Ridder, T. Gehrmann and E. W. N. Glover, Phys. Lett. B 612, 36 (2005) [arXiv:hep-ph/0501291],

[31] A. Gehrmann-De Ridder, T. Gehrmann and E. W. N. Glover, JHEP 0509, 056 (2005) [arXiv:hep-ph/0505111].

[32] M. L. Mangano and S. J. Parke, Phys. Rept. 200, 301 (1991) [arXiv:hepth/0509223].

[33] L. J. Dixon, in QCD and Beyond: TASI 1995, D. E. Soper, ed. (World Scientific, 1996) arXiv:hep-ph/9601359.

[34] L. J. Dixon, E. W. N. Glover and V. V. Khoze, JHEP 0412, 015 (2004) [arXiv:hep-th/0411092].

[35] R. Kleiss and W. J. Stirling, Nucl. Phys. B 262, 235 (1985).

[36] D. A. Kosower, Phys. Rev. D 67, 116003 (2003) [arXiv:hep-ph/0212097].

[37] A. Daleo, T. Gehrmann and D. Maitre, JHEP 0704, 016 (2007) [arXiv:hep$\mathrm{ph} / 0612257]$.

[38] M. P. Le and M. E. Peskin, in preparation.

[39] A. J. Larkoski, M. E. Peskin, Phys. Rev. D81, 054010 (2010). [arXiv:0908.2450 [hep-ph]].

[40] M. E. Peskin, [arXiv:1101.2414 [hep-ph]].

[41] D. A. Kosower, Phys. Rev. D 71, 045016 (2005) [arXiv:hep-ph/0311272]. 
[42] A. Gehrmann-De Ridder, M. Ritzmann, JHEP 0907, 041 (2009). [arXiv:0904.3297 [hep-ph]].

[43] G. Abelof, A. Gehrmann-De Ridder, JHEP 1104, 063 (2011). [arXiv:1102.2443 [hep-ph]].

[44] W. Bernreuther, C. Bogner and O. Dekkers, JHEP 1106, 032 (2011) [arXiv:1105.0530 [hep-ph]].

[45] S. Catani, S. Dittmaier, Z. Trocsanyi, Phys. Lett. B500, 149-160 (2001). [hep$\mathrm{ph} / 0011222]$.

[46] C. Schwinn and S. Weinzierl, JHEP 0704, 072 (2007) [arXiv:hep-ph/0703021].

[47] Y. L. Dokshitzer, V. A. Khoze, S. I. Troian, J. Phys. G G17, 1602-1604 (1991).

[48] V. A. Khoze, J. Ohnemus, W. J. Stirling, Phys. Rev. D49, 1237-1245 (1994). [hep-ph/9308359].

[49] R. Kleiss, W. J. Stirling, S. D. Ellis, Comput. Phys. Commun. 40, 359 (1986).

[50] A. J. Larkoski, M. E. Peskin, Phys. Rev. D83, 034012 (2011). [arXiv:1012.0552 [hep-ph]].

[51] G. Gustafson and A. Nilsson, Z. Phys. C 52, 533 (1991).

[52] J. D. Bjorken, Phys. Rev. D 45, 4077 (1992).

[53] B. Andersson, P. Dahlkvist and G. Gustafson, Phys. Lett. B 214, 604 (1988).

[54] P. Grassberger and I. Procaccia, Phys. Rev. Lett. 50, 346 (1983); P. Grassberger and I. Procaccia, Physica 9D, 189 (1983); F. Takens, in "Atas do 13" (Colóqkio Brasiliero do Matematica, Rio de Janeiro, 1983); J. Theiler, J. Opt. Soc. Am. A, Vol. 7, No 6, 1055 (1990)

[55] S. D. Ellis, Z. Kunszt, D. E. Soper, Phys. Rev. Lett. 69, 3615-3618 (1992). arXiv:hep-ph/9208249. 
[56] M. H. Seymour, Nucl. Phys. B 513, 269 (1998) arXiv:hep-ph/9707338.

[57] W. T. Giele, D. A. Kosower and P. Z. Skands, Phys. Rev. D 84, 054003 (2011) [arXiv:1102.2126 [hep-ph]].

[58] J. R. Walsh and S. Zuberi, arXiv:1110.5333.

[59] G. P. Salam, Eur. Phys. J. C 67, 637 (2010) [arXiv:0906.1833 [hep-ph]].

[60] M. Cacciari, G. P. Salam and S. Sapeta, JHEP 1004, 065 (2010) arXiv:0912.4926.

[61] T. Affolder et al. [CDF Collaboration], Phys. Rev. D 65, 092002 (2002).

[62] See, e.g., R. Field, arXiv:1110.5530.

[63] T. Sjostrand and M. van Zijl, Phys. Rev. D 36, 2019 (1987).

[64] R. P. Feynman, Phys. Rev. Lett. 23, 1415-1417 (1969); K. G. Wilson, Cornell University preprint, November 1970.

[65] M. Bahr et al., Eur. Phys. J. C 58 (2008) 639 arXiv:0803.0883.

[66] M. Bahr, S. Gieseke and M. H. Seymour, JHEP 0807, 076 (2008) arXiv:0803.3633.

[67] M. Bahr, J. M. Butterworth, S. Gieseke and M. H. Seymour, arXiv:0905.4671.

[68] S. Gieseke, D. Grellscheid, K. Hamilton, A. Papaefstathiou, S. Platzer, P. Richardson, C. A. Rohr, P. Ruzicka et al., arXiv:1102.1672.

[69] Available from the Herwig ++ project page: http://projects.hepforge.org/herwig/trac/wiki/MB_UE_tunes/

[70] T. Sjostrand, S. Mrenna, P. Z. Skands, Comput. Phys. Commun. 178, 852-867 (2008) arXiv:0710.3820.

[71] R. Corke, T. Sjostrand, JHEP 1103, 032 (2011) arXiv:1011.1759. 
[72] M. Cacciari, G. P. Salam and G. Soyez, arXiv:1111.6097 [hep-ph].

[73] A. Buckley, J. Butterworth, S. Gieseke, D. Grellscheid, S. Hoche, H. Hoeth, F. Krauss, L. Lonnblad et al., Phys. Rept. 504, 145-233 (2011) arXiv:1101.2599. 